\title{
Iterative Learning Control with Wavelet Filtering
}

\author{
Master's Thesis \\ R.J.E. Merry \\ DCT 2005.54
}

Engineering Thesis Committee:

Prof. Dr. Ir. M. Steinbuch (supervisor)

Dr. Ir. M.J.G. van de Molengraft (coach)

Prof. Ir. O.H. Bosgra

Dr. Ir. M. Heertjes (Philips App. Tech.)

Ir. F.B. Sperling (SKF)

Eindhoven University of Technology

Department of Mechanical Engineering

Control Systems Technology Group

Eindhoven, June 7, 2005 



\section{Summary}

Iterative learning control (ILC) has proven to be a powerful method to derive a high performance feedforward signal for systems that perform repetitive tasks. The feedforward signal is derived through several iterations, based on the tracking error of a feedback controlled system. In practical applications, the tracking error consists of a repetitive part and a non-repetitive part. ILC only compensates for the repetitive part of the error. The non-repetitive part also enters the learning scheme and affects the tracking performance. The problem definition of this thesis is defined as:

Determine how non-repetitive errors affect the tracking error and the learned feedforward signal of ILC and design a method to eliminate the effect of non-repetitive errors.

An expression for the tracking error of an arbitrary iteration is derived as a function of the reference, load disturbances and measurement disturbances. With this expression, the influence of disturbances on ILC can be analyzed. The expression also shows the influence of model uncertainties on the tracking error. The disturbances of the last two iterations appear to have the greatest influence on the tracking error, earlier disturbances only have a limited affect on the tracking error.

In order to reduce the effect of disturbances, three filtering methods are developed. The filtering methods are based on the discrete wavelet transform (DWT). The DWT decomposes a signal into wavelet coefficients in various frequency bands. From these wavelet coefficients, the original signal can be reconstructed again. The DWT performs a local time-frequency decomposition. The nonrepetitive part of the disturbances can be removed by changing the wavelet coefficients, i.e. the frequency content of the signal at specific time instants. The designed wavelet filtering methods calculate a measure for the similarity between two sets of wavelet coefficients at an equal iteration of ILC. The repetitive part of the error results in equal wavelet coefficients, the non-repetitive part in different wavelet coefficients. The repetitive part is identified by adjusting the wavelet coefficients based on thresholding of the similarity measure. The adjusted wavelet coefficients are used to reconstruct a disturbance free error signal, which is the input for ILC.

The wavelet filtering methods differ in the amount of simulations/experiments (runs) required each iteration of ILC and the way the error signals are obtained. The first method uses two runs each iteration. The two error signals are decomposed into two sets of wavelet coefficients which are used to calculated the similarity measure. The second method is recursive and performs only one run each iteration. The second error signal is constructed using the error of the previous iteration and the feedforward update signal of the present iteration, which is linear filtered with a model of the process sensitivity. For the third method, four runs are performed each iteration, which makes it possible to calculate two similarity criteria and to better identify the repetitive part of the error.

In comparison with ILC without wavelet filtering, all proposed wavelet filtering methods lead to smaller tracking errors and to feedforward signals that contain significantly less disturbances. Both deterministic and stochastic disturbances are almost entirely removed by the wavelet filtering methods. The performance of the recursive method depends to a great extend on the quality of the model of the process sensitivity used to construct the second error signal.

The designed wavelet filtering methods make it possible to learn up to higher frequencies where ILC without wavelet filtering becomes dominated by disturbances. The wavelet filtering methods remove the disturbances from the learning scheme, resulting in an improved feedforward and a smaller tracking error after convergence. 


\section{Samenvatting}

Iterative learning control (ILC) kan letterlijk vertaald worden als iteratief lerend regelen. ILC leidt een hoogwaardig feedforward signaal (voorwaartssturing) af voor systemen die herhalende taken uitvoeren. Dit feedforward signaal wordt gedurende enkele iteraties afgeleid door gebruik te maken van de volgfout van een systeem met feedback regeling (terugkoppeling). In de praktijk bestaan volgfouten uit een repeterend deel en een niet-repeterend deel. ILC is in staat alleen voor het repeterende deel van de fout te compenseren. Het niet-repeterende deel komt echter ook in het leeralgoritme van ILC terecht en beïnvloedt de maximaal haalbare prestatie. De probleemstelling van deze scriptie kan als volgt worden omschreven:

Onderzoek hoe niet-repeterende fouten de volgfout en het geleerde feedforward signaal van ILC beïnvloeden en ontwerp een methode die de invloed van de niet-repeterende fouten op het leeralgoritme verwijdert.

In deze scriptie is een uitdrukking afgeleid voor de volgfout van een willekeurige iteratie als functie van het referentiesignaal, ingangsverstoringen (bv. verstoringen op de motor) en uitgangsverstoringen (bv. meetruis). Met behulp van deze uitdrukking kan de invloed van verstoringen op ILC beoordeeld worden. Ook de invloed van modelfouten wordt door de uitdrukking beschreven. Uit de verstoringsanalyse volgt dat de verstoringen van de laatste twee iteraties de grootste invloed hebben op de volgfout. De verstoringen van eerdere iteraties hebben slechts een geringe invloed.

Met behulp van de verkregen kennis uit de verstoringsanalyse zijn filtermethoden ontworpen die het nadelige effect van de niet-repeterende verstoringen verwijderen. Deze filtermethoden zijn gebaseerd op de discrete wavelet transformatie (DWT). De DWT transformeert een signaal in wavelet coëfficiënten in verschillende frequentiegebieden. Met behulp van deze wavelet coëfficiënten kan via een inverse transformatie het oorspronkelijke signaal weer worden verkregen. De DWT maakt het mogelijk om de momentane frequentie-inhoud van een signaal te onderzoeken. Het niet-repeterende gedeelte van de volgfout kan verwijderd worden door de wavelet coëfficiënten aan te passen, dus door de frequentie-inhoud van de volgfout op een bepaald tijdstip te veranderen. De ontworpen filter methoden berekenen een maat voor de overeenkomst tussen twee sets van wavelet coëfficiënten. De twee sets van coëfficiënten worden berekend op dezelfde iteratie van ILC. Het repeterende gedeelte van de volgfout resulteert in gelijke wavelet coëfficiënten, het niet-repeterende deel leidt tot verschillende coëfficiënten. Het niet-repeterende gedeelte wordt verwijderd door de wavelet coëfficiënten te verwijderen waarvan de overeenkomstigheidsmaat een bepaalde drempelwaarde overschrijdt. De aangepaste coëfficiënten worden gebruikt om een foutsignaal zonder verstoringen te reconstrueren. Dit signaal wordt gebruikt als ingang voor het leeralgoritme van ILC.

De ontworpen wavelet filtermethoden verschillen in het aantal simulaties/experimenten (runs) die voor elke iteratie van ILC nodig zijn en in de manier waarop de foutsignalen worden verkregen. De eerste methode voert per iteratie twee runs uit. De twee foutsignalen, die hieruit volgen, worden door de DWT getransformeerd tot twee sets van wavelet coëfficiënten waarmee de overeenkomstigheidsmaat wordt berekend. De tweede methode is een recursieve methode. Deze voert slechts één run per iteratie uit. Het tweede foutsignaal wordt verkregen uit het foutsignaal van de vorige iteratie en de update van het feedforward signaal, welke gefilterd is met een lineair model van de process sensitivity. De derde methode benodigt vier runs per iteratie. Deze methode maakt het 
mogelijk om twee overeenkomstigheidsmaten te berekenen en het repeterende gedeelte van de fout beter te identificeren.

De ontworpen wavelet filtermethoden leiden in combinatie met ILC tot kleinere volgfouten dan het geval is bij ILC zonder wavelet filter. Tevens bevatten de geleerde feedforward signalen met de wavelet filters aanzienlijk minder verstoringen. Zowel deterministische als stochastische verstoringen worden vrijwel geheel verwijderd door de wavelet filtermethoden. De prestatie van de recursieve methode wordt voor een groot deel bepaald door de kwaliteit van het model van de process sensitivity, dat gebruikt wordt om het tweede error signaal te verkrijgen.

De ontworpen wavelet filtermethoden maken het mogelijk om tot hogere frequencies te leren waar ILC zonder wavelet filter gedomineerd wordt door verstoringen. De wavelet filtermethoden verwijderen de verstoringen uit de volgfout en het leeralgoritme van ILC. Dit resulteert in een verbeterd feedforward signaal en een kleinere volgfout na convergentie van ILC. 


\section{Contents}

Summary $\quad$ i

Samenvatting $\quad$ iii

1 Introduction 1

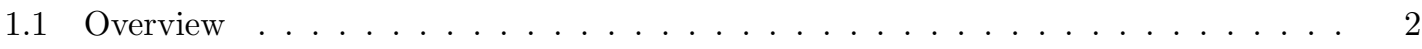

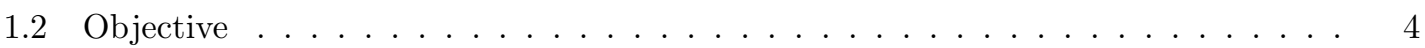

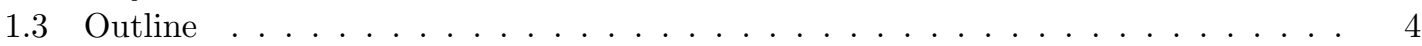

$\begin{array}{lll}2 & \text { Iterative learning control } & 7\end{array}$

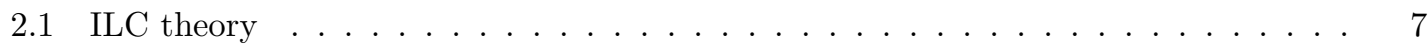

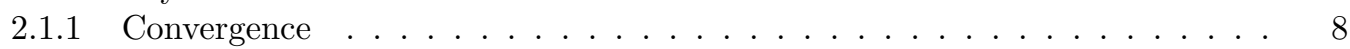

2.1.2 Remaining tracking error . . . . . . . . . . . . . . . . 8

2.2 Disturbance analysis . . . . . . . . . . . . . . . . . . . . 9

2.2 .1 Ideal learning filter . . . . . . . . . . . . . . . . . . . . . . . 9 9

$2.2 .2 \quad$ Approximation learning filter . . . . . . . . . . . . . . . . . 10

2.2.3 Model uncertainties . . . . . . . . . . . . . . . . . . . . . . . . . . . 10

2.3 Discussion . . . . . . . . . . . . . . . . . . . . . 11

3 Wavelet filtering 13

3.1 The discrete wavelet transform . . . . . . . . . . . . . . . . . . . 13

3.2 Wavelet filtering methods . . . . . . . . . . . . . . . . . . . 16

3.2 .1 Wavelet function . . . . . . . . . . . . . . . . . . . 16

3.2 .2 Decomposition level . . . . . . . . . . . . . . . . . . . . . . 17

3.2 .3 Two run method . . . . . . . . . . . . . . . . . . . . 17

3.2 .4 Recursive method . . . . . . . . . . . . . . . . . . . . . . . . . . 18

3.2.5 Higher order method . . . . . . . . . . . . . . . . . . . . . . . 18

$3.2 .6 \quad$ Additional remarks . . . . . . . . . . . . . . . . . . . . . . . . . . . . . . . . . 19

3.3 Discussion . . . . . . . . . . . . . . . . . . . 20

4 Simulations 21

4.1 Simulation model . . . . . . . . . . . . . . . . . . . . . . . . . . 21

4.2 Classic ILC . . . . . . . . . . . . . . . . . . . . . . . 22

4.3 Disturbance analysis . . . . . . . . . . . . . . . . . . 23

4.3 .1 Measurement disturbances . . . . . . . . . . . . . . . . . . . . 24

4.3 .2 Load disturbances . . . . . . . . . . . . . . . . . . . . . 25

4.3 .3 Stochastic disturbances . . . . . . . . . . . . . . . . . . 27

4.3 .4 Model uncertainties . . . . . . . . . . . . . . . . . . . . 27

4.4 Averaging . . . . . . . . . . . . . . . . . . . . . . . . . . 29

4.5 ILC with wavelet filtering $\ldots \ldots \ldots \ldots \ldots \ldots \ldots$

4.5.1 Wavelet filter settings . . . . . . . . . . . . . . . . . . 30

4.5 .2 No disturbances . . . . . . . . . . . . . . . . . . . . 31 
4.5.3 Deterministic disturbances . . . . . . . . . . . . . . . . . . . . . . . . . . .

4.5.4 Stochastic disturbances . . . . . . . . . . . . . . . . . . 36

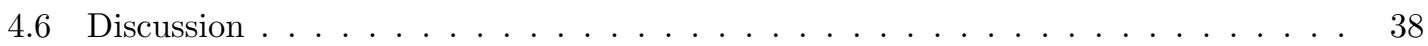

5 Experiments $\quad 41$

5.1 Flexible shaft system . . . . . . . . . . . . . . . . . . . . . . . 41

5.1 Disturbance analysis . . . . . . . . . . . . . . . . . . . . . . . . . . . . . . . . 43

5.1 .2 Applicability of ILC . . . . . . . . . . . . . . . . . . . . . . . . . . . . . . . . . . . . . . .

5.1 .3 Results of ILC . . . . . . . . . . . . . . . . . . . . . . . . . . . . . . . . . . . . . . . .

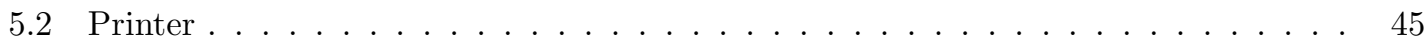

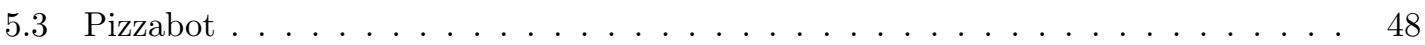

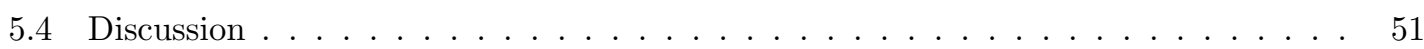

6 Conclusions and recommendations $\quad 53$

6.1 Conclusions . . . . . . . . . . . . . . . . . . . . . 53

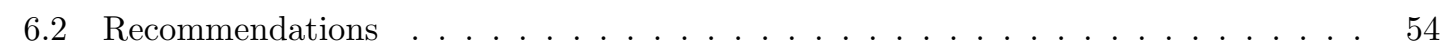

$\begin{array}{lr}\text { Bibliography } & 57\end{array}$

A Zero Phase Error Tracking Control $\quad 61$

B Iterative learning control with wavelet filtering $\quad 63$

C The influence of disturbances in iterative learning control $\quad 67$

$\begin{array}{ll}\text { D Disturbances and model uncertainties in iterative learning control } & 75\end{array}$ 


\section{Chapter 1}

\section{Introduction}

Systems that perform repetitive tasks are likely to make the same errors over and over again. It is possible to teach the system to avoid these errors by giving the system additional input information by means of feedforward control. Every new repetition this feedforward is updated, giving the system a possibility to learn from the errors it made. This method is called iterative learning control (ILC). ILC can derive a high performance feedforward signal for these type of systems.

Two kinds of errors occur when following a reference trajectory; repetitive errors which are identical every iteration and non-repetitive errors which vary every iteration. The derived feedforward signal only compensates for the repetitive part of the error. The non-repetitive errors, e.g. noise and other disturbances, deteriorate the performance of ILC [25, 10]. The design of the learning filters is based on a model of the system $P(s)$ [30], therefore the performance of ILC is also affected by model uncertainties.

The block diagram of a feedback controlled system with ILC added is shown in Fig. 1.1. The system is denoted by $P(s)$, the feedback controller by $C(s)$. The error tracking $e(t)$ is defined as the difference between the reference $r(t)$ and the measured output $y(t)$. The feedforward signal $f(t)$ is derived by ILC based on the error signal $e(t)$. The number of times the reference is tracked and a feedforward update is performed is denoted by the iteration number $k$. The signals of an iteration $k$ are indicated by a subscript, $e_{k}$ being the error of the $k^{t h}$ iteration. The learning filter of ILC is denoted by $L(s)$ and $Q(s)$ is a robustness filter.

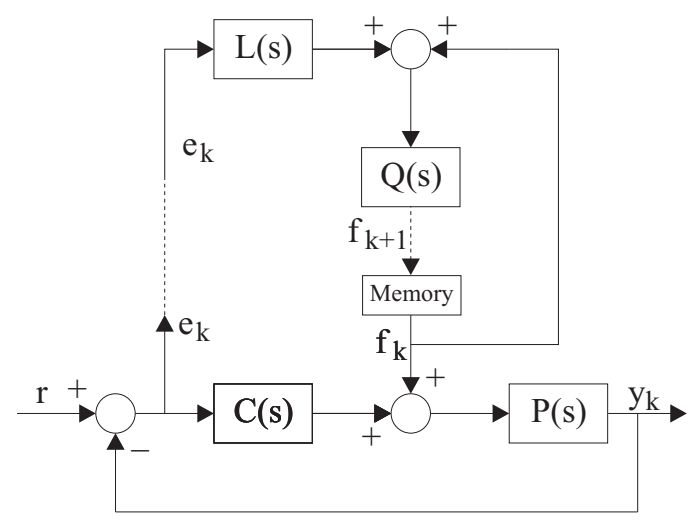

Figure 1.1: block diagram closed loop system with ILC added

The amount of disturbance amplification is influenced by the robustness filter $Q$. The $Q$ filter is usually a low-pass filter since the disturbances and model uncertainties are often present at high frequencies, especially in motion systems. A lower bandwidth of the low-pass $Q$ filter leads to less noise amplification, but decreases the learning performance at high frequencies. 
This thesis focusses on the influence of several kinds of disturbances on the performance of ILC. These disturbances include measurement disturbances, load disturbances and model uncertainties. Methods are developed which reduce the influence of disturbances and result in an improved performance of ILC.

\subsection{Overview}

The first work on ILC is formulated by Uchiyama in 1978 [38]. The idea proposed by Uchiyama was developed further by Arimoto in the mid eighties [2]. He proposed the first ILC scheme. Since then, ILC studies have been performed for various types of systems and applications.

The presence of disturbances in ILC received some attention in the past. A disturbance analysis of ILC is performed in [26] for the open-loop case with measurement disturbances only. In [24], a feedback loop is included in the analysis. The performance of the learning algorithm is evaluated by the output of the feedback controller for the case of no measurement disturbances. In [25, 35] a recursive formulation of the tracking error is used in combination with a disturbance free error signal obtained without any ILC input. Furthermore, the load disturbances are assumed to be repetitive over the iterations. The influence of model uncertainties is investigated in [9] for the lifted ILC approach and constant model uncertainties only. In this thesis, all kind of errors, i.e. measurement, load and model errors, are taken into account for a feedback controlled system. The contribution of this thesis to the field of disturbance analysis in ILC is depicted in Table 1.1.

Table 1.1: Overview of disturbance analyses found in literature

\begin{tabular}{c|c|c|c|c|c|c} 
& $\begin{array}{c}\text { Dijkstra } \\
{[10]}\end{array}$ & $\begin{array}{c}\text { Norrlöf } \\
{[24]}\end{array}$ & $\begin{array}{c}\text { Norrlöf } \\
{[25]}\end{array}$ & $\begin{array}{c}\text { Panzieri } \\
{[26]}\end{array}$ & $\begin{array}{c}\text { Tinsel } \\
{[35]}\end{array}$ & This thesis \\
\hline $\begin{array}{c}\text { Classic ILC } \\
\text { Lifted ILC }\end{array}$ & $\mathrm{x}$ & $\mathrm{x}$ & $\mathrm{x}$ & $\mathrm{x}$ & $\mathrm{x}$ & $\mathrm{x}$ \\
\hline $\begin{array}{c}\text { Open loop } \\
\text { Closed loop }\end{array}$ & $\mathrm{x}$ & $\mathrm{x}$ & $\mathrm{x}$ & $\mathrm{x}$ & & $\mathrm{x}$ \\
\hline $\begin{array}{c}\text { Measurement dist. } \\
\text { Load dist. }\end{array}$ & $\mathrm{x}$ & $\mathrm{x}$ & $\mathrm{x}$ & $\mathrm{x}$ & $\mathrm{x}$ & $\mathrm{x}$ \\
Model uncertainties & $\mathrm{x}$ & $\mathrm{x}$ & $\mathrm{x}$ & & $\mathrm{x}$ & $\mathrm{x}$ \\
\hline $\begin{array}{c}\text { Recursive formulation } \\
\text { Non-recursive form. }\end{array}$ & & $\mathrm{x}$ & $\mathrm{x}$ & $\mathrm{x}$ & $\mathrm{x}$ & $\mathrm{x}$ \\
\hline
\end{tabular}

Below, a short overview is given of the methods found in literature which improve the performance of ILC in the presence of disturbances. Noise suppression for the lifted ILC representation is preformed in $[3,10,37]$. In the lifted representation the system dynamics are described as a static map and the learning dynamics are described by difference equations in the iteration domain. In the lifted representation, the reference trajectory $r$ is considered as a disturbance that does not change from iteration to iteration and needs to be suppressed. The internal model principle (IMP) [14] states that disturbances which are constant from iteration to iteration can be generated by an autonomous system. A model of the disturbance generating system is added to the controller. Controllable inputs are added to this model. The IMP provides asymptotic rejection of constant disturbances and can improve the tracking results of ILC [4]. For the lifted representation, the suppression of noise can be done by a multi-objective design optimization approach [37] or by buffer-state ILC which uses a linear quadratic objective and a constraint on the noise amplification [11].

An ILC algorithm for systems which are subject to measurement noise is introduced in [23]. The algorithm is based on a multi-loop control interpretation of the signal flow in ILC to derive the necessary and sufficient condition for convergence. The input is updated according to an estimate of the Markov parameters [23]. The presented algorithm incorporates only measurement noise. 
A phase lead compensated ILC scheme is presented in [40], it has a significantly broader learnable frequency band, which results in a higher precision. For the learning filter, an inverse system model has to be calculated, which will amplify high frequency noise, especially in motion systems which are minimum phase systems with negative phase. An alternative is using the inverse of the resonance mode of the system (partial system inverse). Two different learning gain matrices are used in [40] to phase lead filter the error, a transpose of the system's Markov parameter matrix and a matrix based on the inverse plant phase characteristics using the inverse Fourier transform. Phase compensated ILC does not suffer from increasing errors during the iteration process and is robust against modeling errors.

Knowledge of the behavior and characteristics of disturbances can be used to improve the performance of ILC. If the pattern of the non-repetitive disturbances is not known, a disturbance observer in the iteration domain can be used to improve the performance of the ILC scheme [4].

Classic ILC does not account for position dependent dynamics, setpoint trajectory changes and stochastic effects. These shortcomings can be improved using time-frequency analysis of the feedforward signal $[28,41]$. The high energy of the error signal is concentrated very locally in time, the learning process outside these intervals leads to an amplification of the present noise. A timevarying robustness filter can adapt to the momentary frequency content of the feedforward signal and allow high frequency system dynamics at the appropriate time instants. A time-varying $Q$ filter can be designed using a time-frequency analysis [13, 42, 41]. The time-frequency analysis of [13] is performed using the Wigner-Ville decomposition (WVD). The WVD is a global transform method and can be thought of as an autopowerspectrum estimate as a function of time or an autocorrelation estimate as a function of frequency. The WVD can show negative energy levels and cross terms, which are physically irrelevant. The analysis results of the WVD are often difficult to interpret and the amount of calculations needed for the analysis exceeds the STFT and WT significantly. The inverse WVD is not workable and very redundant [29]. The time-frequency analysis for adjusting the bandwidth of the $Q$ filter can also be done using the wavelet transform [42]. The bandwidth of the $Q$ filter is updated iteratively based on the magnitude of the error as a function of time during each iteration.

All methods change the ILC algorithm to suppress the influence of disturbances. If the disturbances can be kept from entering the ILC scheme, adjustment of the ILC algorithm is not necessary anymore, i.e. we tackle the disturbances at the source signal for ILC, the tracking error. In this thesis, filtering is applied to the error which keeps the disturbances from entering the learning scheme of ILC. A schematic overview of the disturbance reduction methods in ILC is shown in Fig. 1.2.

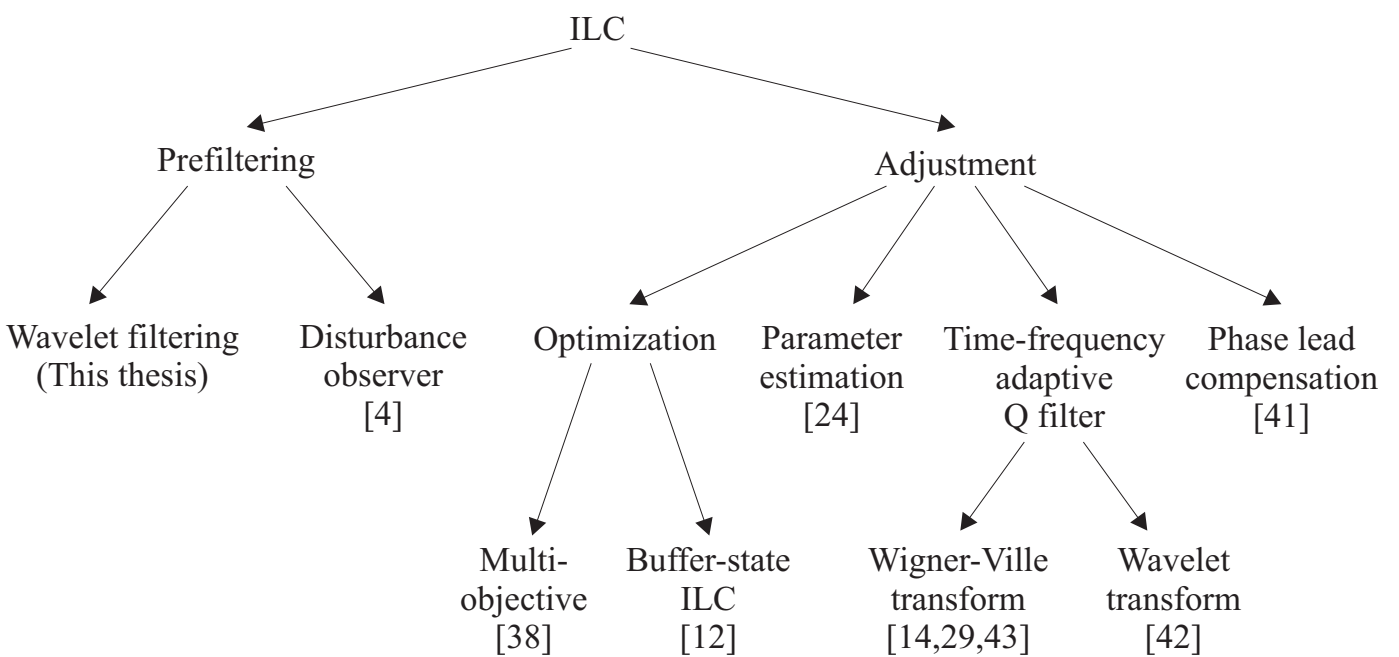

Figure 1.2: Schematic overview of ILC disturbance reduction methods found in literature 


\subsection{Objective}

A standard feedback control scheme is shown in Fig. 1.3. In the feedback control scheme, load disturbances $n(t)$ and measurement disturbances $d(t)$ are present. The disturbances contribute to the non-repetitive part of the error, i.e. they differ every iteration of ILC. The tracking error $e(t)$ can be written as

$$
e=\underbrace{\frac{1}{1+P C}}_{S} r-\underbrace{\frac{1}{1+P C}}_{S} d-\underbrace{\frac{P}{1+P C}}_{S_{P}} n .
$$

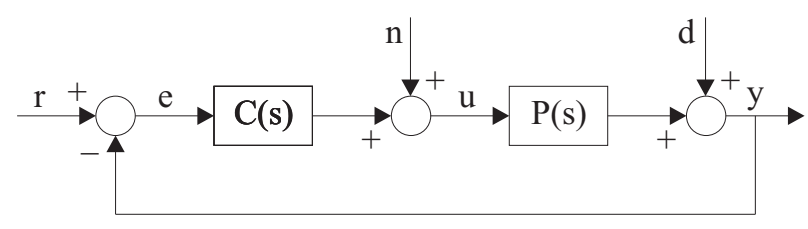

Figure 1.3: Feedback control scheme with measurement disturbances $d$ and load disturbances $n$

It can be seen that load disturbances $n$ appear in the error filtered by the process sensitivity $S_{P}$. Output disturbances $d$ appear in the tracking error filtered by the sensitivity $S$. The learning of the feedforward in ILC is based on the tracking error $e(t)$. Since the tracking error contains the disturbances, these disturbances will also enter the learning scheme of ILC and the learned feedforward signal. The learned feedforward signal with the non-repetitive disturbances will affect the control input of the next iteration and with this the error of the next iteration. This results in the following problem definition:

Determine how non-repetitive errors affect the tracking error and the learned feedforward signal of ILC and design a method to eliminate the effect of non-repetitive errors.

In order to investigate the influence of the non-repetitive errors on ILC, first the theory of ILC is addressed. A disturbance analysis will be performed which will show the results of both measurement and load disturbances as well as model uncertainties on the tracking error of ILC.

The obtained knowledge about the influence of non-repetitive disturbances can be used to design filtering methods which remove the non-repetitive part of the error. In order to distinguish between the repetitive and non-repetitive part of the error, a time-frequency analysis method will be used on the basis of wavelet analysis. The wavelet transform makes it possible to analyze the momentary frequency content of signals. The designed wavelet filtering methods perform a filtering on the tracking error signal and the resulting filtered error is used as input for ILC.

The performance of ILC with wavelet filtering methods is validated and compared to the performance of classic ILC by means of both simulations and experiments. The advantages and disadvantages of the proposed methods are compared to the performance of classic ILC, i.e. without wavelet filtering.

\subsection{Outline}

The ILC concept will be described in more detail in Chapter 2. The effect of disturbances in ILC is studied in Chapter 2, where an expression for the tracking error of an arbitrary iteration as a function of the reference $r$ and the various disturbances will be derived. With the knowledge of the disturbance analysis, three different wavelet filtering methods will be designed. The wavelet filtering methods are based on the discrete wavelet transform (DWT). The DWT, wavelet filtering methods and incorporation of the filtering methods in ILC will be treated in Chapter 3 . The derived error expression of Chapter 2 will be validated by means of simulations in Chapter 4 . Furthermore, simulations will be performed with and without the designed wavelet filtering methods. 
In Chapter 5, the wavelet filtering methods will be tested by means of experiments. Experiments will be performed on three different systems, an academic system and both a relatively simple and a complex industrial system. Finally, conclusions will be drawn and recommendations for future research will be given in Chapter 6 . 


\section{Chapter 2}

\section{Iterative learning control}

ILC is an efficient method to derive a high performance feedforward signal for systems which follow the same trajectory repeatedly. Two kind of errors occur when following a trajectory, repetitive errors which are equal every run and non-repetitive errors that vary every run. The derived feedforward signal of ILC only compensates for the repetitive part of the error. At the same time the non-repetitive errors deteriorate the performance of ILC [25, 10].

The theory of ILC will be described in Section 2.1. The design of the learning filters and convergence properties of ILC will be presented. In Section 2.2, a general equation will be derived which describes the influence of measurement and load disturbances on the tracking error of ILC. The influence of model uncertainties on the tracking error will also be discussed in Section 2.2.

\subsection{ILC theory}

ILC reduces the repetitive part of the error signal during several subsequent runs with a feedforward update, called iterations [30]. All iterations have an equal finite-time length $t \in\left[t_{0}, t_{e}\right]$. A block diagram of a feedback controlled system with ILC added is shown in Fig. 2.1 in the presence of both measurement disturbances $d(t)$ and load disturbances $n(t)$.

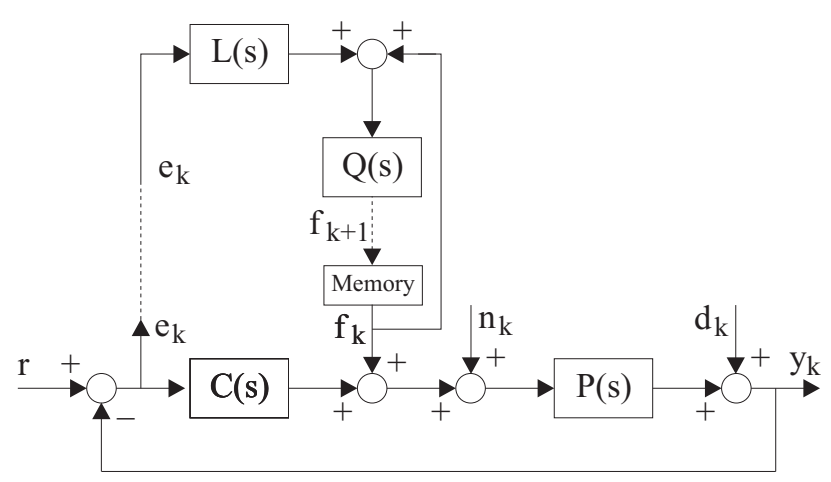

Figure 2.1: control scheme ILC

ILC uses the tracking error off-line to calculate a feedforward signal that reduces the error between subsequent iterations. For this, the error $e_{k}$ is filtered by a learning filter $L(s)$ and added to the feedforward $f_{k}$. The sum of the filtered error and the feedforward is filtered by a robustness filter $Q(s)$ to obtain the feedforward signal of the next iteration $f_{k+1}$. The performance of ILC is determined by the design of the filters $L(s)$ and $Q(s)$. 
The feedback error with respect to the reference $r$ and the feedforward signal of the $k^{t h}$ iteration can be written as

$$
e_{k}=S r-S_{P} f_{k}
$$

The learning update from one iteration to the next is

$$
f_{k+1}=Q\left(f_{k}+L e_{k}\right)
$$

Using (2.1) and (2.2) the error of iteration $k+1$ can be written as

$$
e_{k+1}=Q\left(1-L S_{P}\right) e_{k}
$$

\subsubsection{Convergence}

If the error reduces between subsequent iterations, the ILC scheme converges. From (2.3), the error can be seen to converge if

$$
\left|Q\left(1-L S_{P}\right)\right|<1, \quad \forall s \in j \mathbb{R} .
$$

Eq. (2.4) is based on the fixed point theorem [13] and gives a sufficient, but not necessary condition for the convergence of ILC. As can be seen in (2.3), the error becomes zero after one iteration if the learning filter $L$ equals the inverse $S_{P}$, this is the dead beat solution. However, the ideal $L$ filter is difficult to derive since the inverse $S_{P}$ is often a non-causal transfer function, especially for motion systems. Another difficulty arises if $S_{P}$ is non-minimum phase, the inverse of $S_{P}$ will then have instable poles and numerical problems can arise. Therefore, in practical cases an approximated $S_{P}^{-1}$ is used.

The $Q$ filter provides robustness against modeling errors. The $Q$ filter is designed in such a way that the convergence criterion is satisfied for all frequencies where $\left|1-S_{P} L\right|>1$. The pass-band gain of the $Q$ filter is critical for the tracking performance. Another important requirement of the $Q$ filter is that it does not affect the phase of the filtered signal. The zero phase characteristic is obtained by filtering the signal with the forward-backwards method (filtfilt.m in Matlab).

The Q filter compensates for the mismatch between the learning filter and the system dynamics and for the effect of nonlinear dynamics. These mismatches appear mainly at high frequencies. Therefore, the $Q$ filter is often chosen as a low-pass filter.

\subsubsection{Remaining tracking error}

If it is assumed that the ILC scheme converges for $k \rightarrow \infty$ the feedforward signal $f_{k}=f_{k-1}$ and the error $e_{k}=e_{k-1}$, the learning rule of (2.2) can be written as

$$
\begin{aligned}
\lim _{k \rightarrow \infty} f_{k} & =Q\left(f_{k}+L e_{k}\right) \\
& =\frac{Q L}{1-Q} e_{k} .
\end{aligned}
$$

Combining this result with the error equation of (2.1) gives

$$
\begin{aligned}
\lim _{k \rightarrow \infty} e_{k} & =\frac{1}{1+P C} r-\frac{P}{1+P C}\left(\frac{Q L}{1-Q} e_{k}\right) \\
& =\left(1+\frac{P Q L}{(1+P C)(1-Q)}\right)^{-1} \frac{1}{1+P C} r \\
\lim _{k \rightarrow \infty} e_{k} & =\frac{1-Q}{(1-Q)(1+P C)+P Q L} r .
\end{aligned}
$$

From (2.6), one can see that the use of a $Q$ filter limits the minimal achievable tracking error for $k \rightarrow \infty$ The ILC scheme will never converge to a zero tracking error in the frequency range where $Q \neq 1$. 


\subsection{Disturbance analysis}

The ILC concept assumes that the disturbances, system dynamics, uncertainties and the trajectories are iteration-independent. However, there are also non-repetitive errors which vary every iteration, e.g. measurement and load disturbances. These non-repetitive errors limit the performance of the ILC scheme.

To determine how the disturbances affect the performance of ILC, an expression for the error of iteration $k$ as a function of the reference signal $r$ and the disturbances $n_{k}$ and $d_{k}$ of the current and previous iterations is derived. The error of iteration $k$ can be written as

$$
e_{k}=S r_{k}-S d_{k}-S_{P} n_{k}-S_{P} f_{k} .
$$

The feedforward of iteration $k$ can be written with use of the error $e_{k-1}$ and (2.2) as

$$
\begin{aligned}
f_{k} & =Q\left(f_{k-1}+L\left(S r-S d_{k-1}-S_{P} n_{k-1}-S_{P} f_{k-1}\right)\right) \\
& =Q\left(I-L S_{P}\right) f_{k-1}+Q L S r-Q L S d_{k-1}-Q L S_{P} n_{k-1} .
\end{aligned}
$$

Substitution of (2.8) in (2.7) now results in

$$
\begin{aligned}
e_{k} & =S r-S d_{k}-S_{P} n_{k}-S_{P}\left(Q\left(I-L S_{P}\right) f_{k-1}+Q L S r-Q L S d_{k-1}-Q L S_{P} n_{k-1}\right) \\
& =-S_{P} Q\left(I-L S_{P}\right) f_{k-1}+\left(I-S_{P} Q L\right) S r-S d_{k}+S_{P} Q L d_{k-1}-S_{P} n_{k}+S_{P}^{2} Q L n_{k-1}
\end{aligned}
$$

By repeating the steps taken in (2.7) to (2.9), the error of an arbitrary iteration $k$ can be written as a function of the reference $r$ and the disturbances of the previous iterations. The total tracking error of iteration $k$ now becomes

$$
\begin{aligned}
e_{k}= & S r-\sum_{j=1}^{k-1} S_{P} Q\left(Q\left(1-L S_{P}\right)\right)^{k-j-1} L S r \\
& -S d_{k}+S_{P} Q L S d_{k-1}+\sum_{j=1}^{k-2} S_{P} Q\left(Q\left(1-L S_{P}\right)\right)^{k-j-1} L S d_{j} \\
& -S_{P} n_{k}+S_{P}^{2} Q L n_{k-1}+\sum_{j=1}^{k-2} S_{P}^{2} Q\left(Q\left(1-L S_{P}\right)\right)^{k-j-1} L n_{j} .
\end{aligned}
$$

The reference $r$ is not varied over the iteration process and contributes to the tracking error $e_{k}$ through the filter $\left(1-S_{P} Q L\right) S$ and the first summation term of (2.10). The measurement and load disturbances of iteration $k$ are filtered by respectively $S$ and $S_{P}$, the disturbance signals of iteration $k-1$ by are filtered by $S_{P} Q L S$ for $d_{k-1}$ and $S_{P}^{2} Q L$ for $n_{k-1}$. Measurement and load disturbances of older iterations are filtered by the second an third summation terms of (2.10). The presence of the disturbances in the learning process results in a degraded performance of ILC, i.e. an increase of the tracking error.

\subsubsection{Ideal learning filter}

If an ideal learning filter, i.e. $L=S_{P}^{-1}$, can be derived, no $Q$ filter is required since the convergence criterion of $(2.4)$ is already met $(Q(s)=1)$. The tracking error of $(2.10)$ then reduces to

$$
e_{k}=-S d_{k}+S d_{k-1}-S_{P} n_{k}+S_{P} n_{k-1} .
$$

The tracking error only depends on the disturbances of the last two iterations. In the worst case scenario, the load and measurement disturbances have an opposite sign with respect to the last two iterations. For an ideal learning filter the error can become at most a factor two larger. In case of no disturbances and an ideal learning filter, the tracking error $e_{k}$ becomes zero. 


\subsubsection{Approximation learning filter}

If $L \approx S_{P}^{-1}$, a $Q$ filter is generally needed. The error $e_{k}$ depends on the disturbances of all previous iterations. In comparison to the filters of the disturbance signals of iteration $k-1$, the filters of disturbances of older iterations have an additional multiplication with the convergence criterion a number of times. The number of multiplications depends on the iteration number as can be seen in (2.10). The summation terms converge to fixed transfer functions in the frequency domain for $k \rightarrow \infty$ since the convergence criterion of (2.4) has an amplitude smaller than one for all frequencies.

The magnitudes of the Frequency Response Functions (FRFs) of the summation terms are smaller than the magnitudes of the FRFs of the disturbance filters for iterations $k$ and $k-1$. Disturbances of iterations older than the last two are expected to have a smaller contribution to the error than the disturbances of the last two iterations. The amplification of the error by the presence of disturbances is expected to me maximally a bit larger than a factor two.

\subsubsection{Model uncertainties}

The learning filter $L$ is derived from a model of the process sensitivity $S_{P}$. A widely used method to obtain the model of $S_{P}$ is by using a fit of a FRF measurement of the system $P$ and by using the designed feedback controller $C$. The fitted FRF of the system $P$ will not be an exact representation of the real system. Therefore we say that the system model $\tilde{P}$ has a model uncertainty $\Delta P$ with respect to the real system $P$, which we model as an additive model uncertainty

$$
\tilde{P}=P+\Delta P .
$$

The process sensitivity $S_{P}$ used for the design of the $L$ filter incorporates the model uncertainties as

$$
\tilde{S}_{P}=\frac{\tilde{P}}{1+\tilde{P} C}
$$

This results in a learning filter which contains model uncertainties as

$$
\tilde{L}=\tilde{S}_{P}^{-1} .
$$

The influence of model uncertainties on the tracking error of ILC can be described by replacing the learning filter $L$ in $(2.10)$ by the learning filter with model uncertainties $\tilde{L}$ as

$$
\begin{aligned}
e_{k}= & S r-\sum_{j=1}^{k-1} S_{P} Q\left(Q\left(1-\tilde{L} S_{P}\right)\right)^{k-j-1} \tilde{L} S r \\
& -S d_{k}+S_{P} Q \tilde{L} S d_{k-1}+\sum_{j=1}^{k-2} S_{P} Q\left(Q\left(1-\tilde{L} S_{P}\right)\right)^{k-j-1} \tilde{L} S d_{j} \\
& -S_{P} n_{k}+S_{P}^{2} Q \tilde{L} n_{k-1}+\sum_{j=1}^{k-2} S_{P}^{2} Q\left(Q\left(1-\tilde{L} S_{P}\right)\right)^{k-j-1} \tilde{L} n_{j}
\end{aligned}
$$

It can be seen that the influence of the disturbances of the last iteration $k$ on the tracking error $e_{k}$ is not affected by the model uncertainties. This is expected since the disturbances of the last iteration have not entered the ILC scheme and are not yet filtered by the learning filter.

Note that the convergence criterion must be evaluated using the learning filter with model uncertainties and the process sensitivity calculated with the measured system $P$ and the designed controller $C$. The convergence criterion is still required to hold, i.e. $\left|Q\left(1-\tilde{L} S_{P}\right)\right|<1$. 


\subsection{Discussion}

In this chapter, the ILC concept has been explained in more detail. Furthermore a disturbance analysis has been performed which shows the effect of measurement disturbances, load disturbances and model uncertainties on the tracking error of ILC.

The learning filter of ILC has been discussed for a system with feedback control loop. Without a feedback control loop, the optimal learning filter $L$ is no longer an inverse of $S_{P}$ but an inverse $P$. The same problems of non-causality and non-minimum phase zeros can occur in this case, invoking the need for an approximation of the inverse.

The present position of the $Q$ filter in the ILC scheme (see Fig. 2.1) causes the feedforward of the present iteration $f_{k}$ to be filtered also, whereas this feedforward signal was already filtered in the previous iteration. Placing the $Q$ filter directly after the learning filter $L$ leads to the following convergence criterion

$$
\left|1-Q L S_{P}\right|<1
$$

The original position of the $Q$ filter makes it possible to adjust the whole criterion $1-L S_{P}$. With the new position of the $Q$ filter only the term $L S_{P}$ can be altered by the $Q$ filter. The convergence criterion can be represented using vectors in the complex plane, as shown in Fig. 2.2 for two different situations. The convergence criterion is met if the amplitude of the vector between the vector $L S_{P}$ and the unity vector is smaller than one. For the old configuration, the convergence criterion can be met in both situations by adjusting the amplitude of $1-L S_{P}$ using the $Q$ filter. In the new configuration only the vector $L S_{P}$ can be changed. The convergence criterion of the situation in the left figure can be met by changing only the amplitude of $L S_{P}$. For the situation in the right, both the amplitude and phase of $L S_{P}$ have to be altered in order to obtain a vector $1-L S_{P}$ with an amplitude smaller than one. If the vector $L S_{P}$ is located in the left half plane, the convergence criterion can only be met in the new configuration if the phase of $L S_{P}$ is altered by the $Q$ filter.
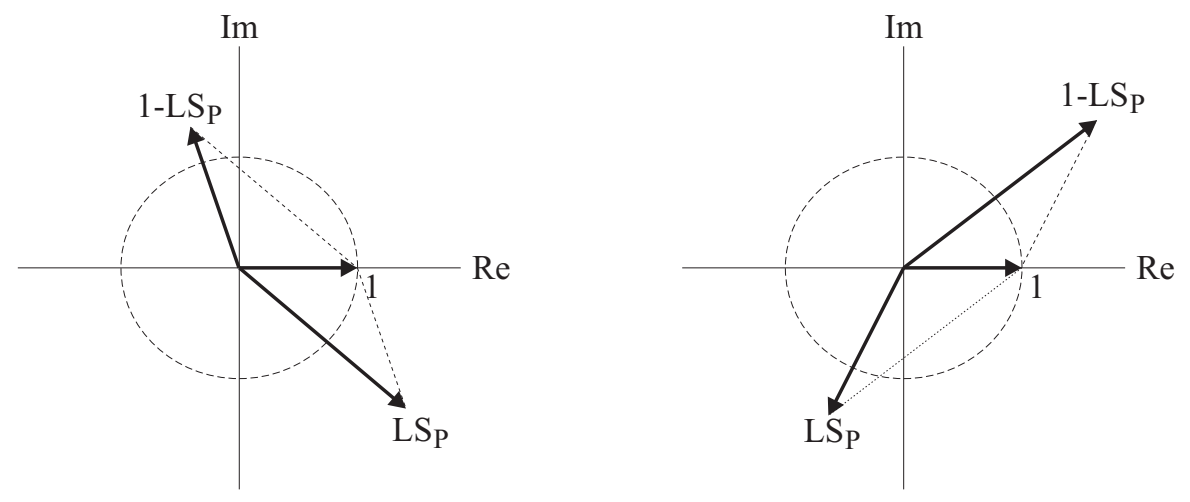

Figure 2.2: Vector representation convergence criterion in the complex plane

With the new position of the $Q$ filter it is possible to obtain convergence, however the design of the $Q$ filter is more difficult since both amplitude and phase have to be designed. For the original position of the $Q$ filter, only the amplitude has to be designed.

The derived error equation of Section 2.2 shows the influence of disturbances of all iterations and model errors on the tracking error $e_{k}$. The tracking error is determined by a great extend by the disturbance of the last two iterations. Older disturbances only give a small contribution to the error. The performance of ILC can be reduced by approximately a factor two if the non-repetitive part of the disturbances is kept out of the learning scheme. For this a filtering method is designed which removes the non-repetitive part of the tracking error. This filtering method is based on wavelet analysis and will be discussed in the next chapter. 


\section{Chapter 3}

\section{Wavelet filtering}

The disturbance analysis of ILC has shown that the disturbances present in the learning scheme deteriorate the performance. In order to eliminate the disturbances a filtering method will be designed which is based on the wavelet transform (WT). The continuous wavelet transform (CWT) calculates the convolution between a signal and a wavelet function. A wavelet function is a localized oscillating function which contains both the analyzing function and the window. The time information is obtained by shifting the wavelet function over the signal. The frequencies are altered by contraction and dilatation of the wavelet function. The wavelet filters act as bandpass filters in the frequency domain. The discrete wavelet transform (DWT) uses filter banks to decompose a signal into wavelet coefficients. These wavelet coefficients represent the signal content in various frequency bands.

The time-frequency decomposition of the error signals makes it possible to analyze and change the momentary frequency content of the error signals. By adjusting the wavelet coefficients, the reconstructed signal of the synthesis filter bank can be changed in comparison to the original signal. This gives the DWT some attractive properties over linear filtering. Compared to the CWT, the DWT is easier to compute and the wavelet coefficients are easier to interpret. The designed wavelet filtering methods remove the non-repetitive part of the error, based on a similarity measure of multiple sets of wavelet coefficients and thresholding.

The discrete wavelet transform will be shortly explained in Section 3.1. Three different wavelet filtering methods will be presented in Section 3.2. All wavelet filtering methods are based on the DWT.

\subsection{The discrete wavelet transform}

Wavelet analysis is a time-frequency technique which is especially useful for analyzing non-stationary signals. In this section, a short introduction of the discrete wavelet transform (DWT) is given. A more detailed description of wavelet analysis and of the DWT can be found in [31, 20]. The DWT uses an analysis filter bank to decompose a signal into wavelet coefficients at various frequency bands. The wavelet coefficients can be used in a synthesis filter bank to recompose the original signal. The wavelet coefficients represent the signal content in various frequency bands as a function of time.

An example of a three level analysis and synthesis filter bank is shown in Fig. 3.1. The discrete time signal $x(k)$ is filtered in the analysis bank by the wavelet filters $L(z)$ and $H(z)$ which separate the frequency content in frequency bands of equal width. The filters $L(z)$ and $H(z)$ can be considered as low-pass and high-pass analysis and synthesis filters. Because the signal length is doubled after the filters and the amount of information remains unchanged, downsampling by a factor two, denoted in Fig. 3.1(a) by $(\downarrow 2)$, is performed. The downsampling removes the odd-numbered components of the filter output. 
Wavelet filters with equal properties as wavelet functions can be obtained by an iteration of filters with rescaling. The sequences of filters and downsampling in the various branches of the filter bank represent the frequency content of a wavelet function at a specific scale, i.e. they act as a band-pass filter in the frequency domain. The wavelet filters can be classified into two classes, orthogonal and biorthogonal wavelets. For the DWT special families of wavelet filters are designed according to certain design rules, leading to the four filters [29, 31, 20].

The filter bank can be expanded to an arbitrary level, depending on the desired resolution. At each step, the output of the filter $L(z)$ is decomposed further, leading to a better frequency resolution at lower frequencies. Each step improves the frequency resolution by a factor two. The time resolution is however halved because of the downsampling.

From the wavelet coefficients the time signal can be reconstructed by a synthesis filter bank. In the synthesis filter bank the wavelet coefficients are upsampled again $(\uparrow 2)$, i.e. zeros are placed in between the wavelet coefficients.

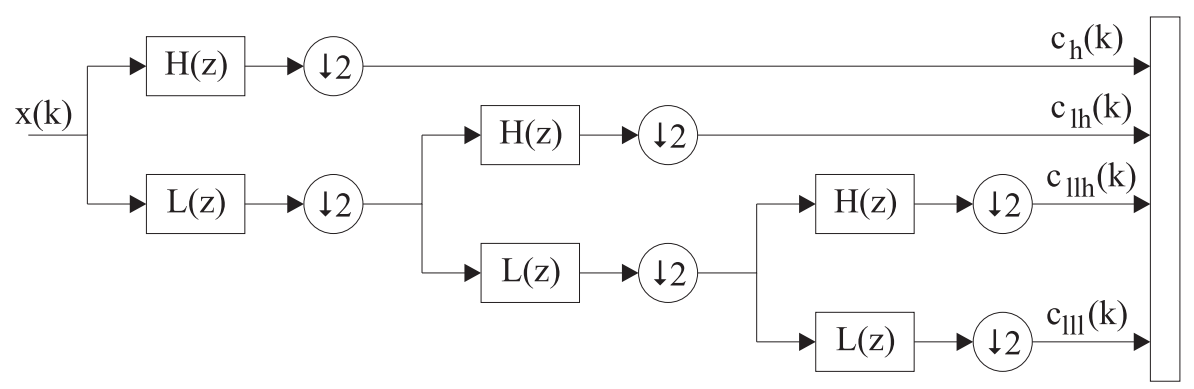

(a) analysis filter bank

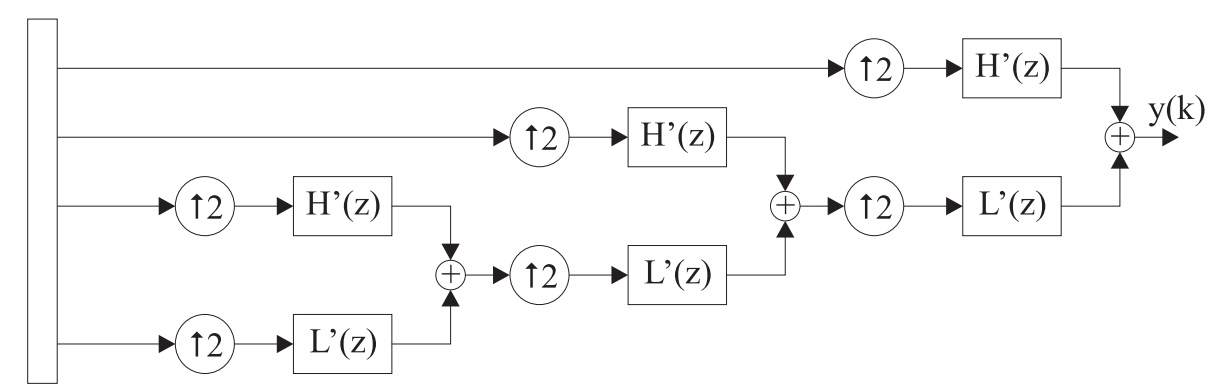

(b) synthesis filter bank

Figure 3.1: Three level filter bank

The different output signals of the filter bank are called subbands, the filter-bank technique is therefore also known as subband coding [29]. The branch with only low-pass filters $L(z)$ retrieves the approximation of the signal. The coefficients of this branch are called approximation coefficients $c A$. Detail information of the signal at various frequency bands is extracted by the other branches of the filter bank, the coefficients are called detail coefficients $c D$.

The succes of a certain decomposition depends strongly on the chosen wavelet function, depending on the signal characteristics. The various wavelet filter families and their properties are described in $[29,20]$.

For a $p$-level decomposition the highest frequency observed in the approximation subband can be calculated as a function of the sampling frequency $f_{s}$ as

$$
f_{c A, \max }=\frac{f_{s}}{2^{p+1}} .
$$


The frequency content of the approximation $c A$ and detail frequency bands $c D$ can be calculated as

$$
\begin{aligned}
f_{c A} & =\left[\begin{array}{ll}
0, & 2^{-p-1} f_{s}
\end{array}\right] \\
f_{c D_{p}} & =\left[\begin{array}{ll}
2^{-p-1} f_{s}, & 2^{-p} f_{s}
\end{array}\right] .
\end{aligned}
$$

An example of a DWT is shown in Fig. 3.2. The top figure shows the time signal $x(t)$ which consists of a sine with a frequency of $45 \mathrm{~Hz}$ from $0.1 \mathrm{~s}$ up to $0.3 \mathrm{~s}$, at $0.2 \mathrm{~s}$ the signal contains a pulse. At $0.4 \mathrm{~s}$ the signal shows a sinusoid with a frequency of $250 \mathrm{~Hz}$ which changes to $75 \mathrm{~Hz}$ at $0.5 \mathrm{~s}$. The time interval from $0.7 \mathrm{~s}$ up to $0.9 \mathrm{~s}$ consists of two superposed sinusoids with frequencies of $30 \mathrm{~Hz}$ and $110 \mathrm{~Hz}$. The signal is sampled at a frequency of $1 \mathrm{kHz}$. The DWT is performed with a Daubechies wavelet function with order 4 ('db4', see [20]) and a three level filter bank. There exists a trade-off between the order of the wavelet function and the computation time. Higher order wavelets are better able to distinguish between the various frequencies, but require more computation time $[6,20]$. The signal content in the various frequency subbands is shown in the lowest four figures of Fig. 3.2. The frequency ranges of the subbands are given in Table 3.1.

\begin{tabular}{|c|c|c|}
\hline subband & $f_{\text {low }} \mathrm{Hz}$ & $f_{\text {high }} \mathrm{Hz}$ \\
\hline$c_{l l l}$ & 0 & 62.5 \\
$c_{l l h}$ & 62.5 & 125 \\
$c_{l h}$ & 125 & 250 \\
$c_{h}$ & 250 & 500 \\
\hline
\end{tabular}

Table 3.1: Frequency content subbands DWT
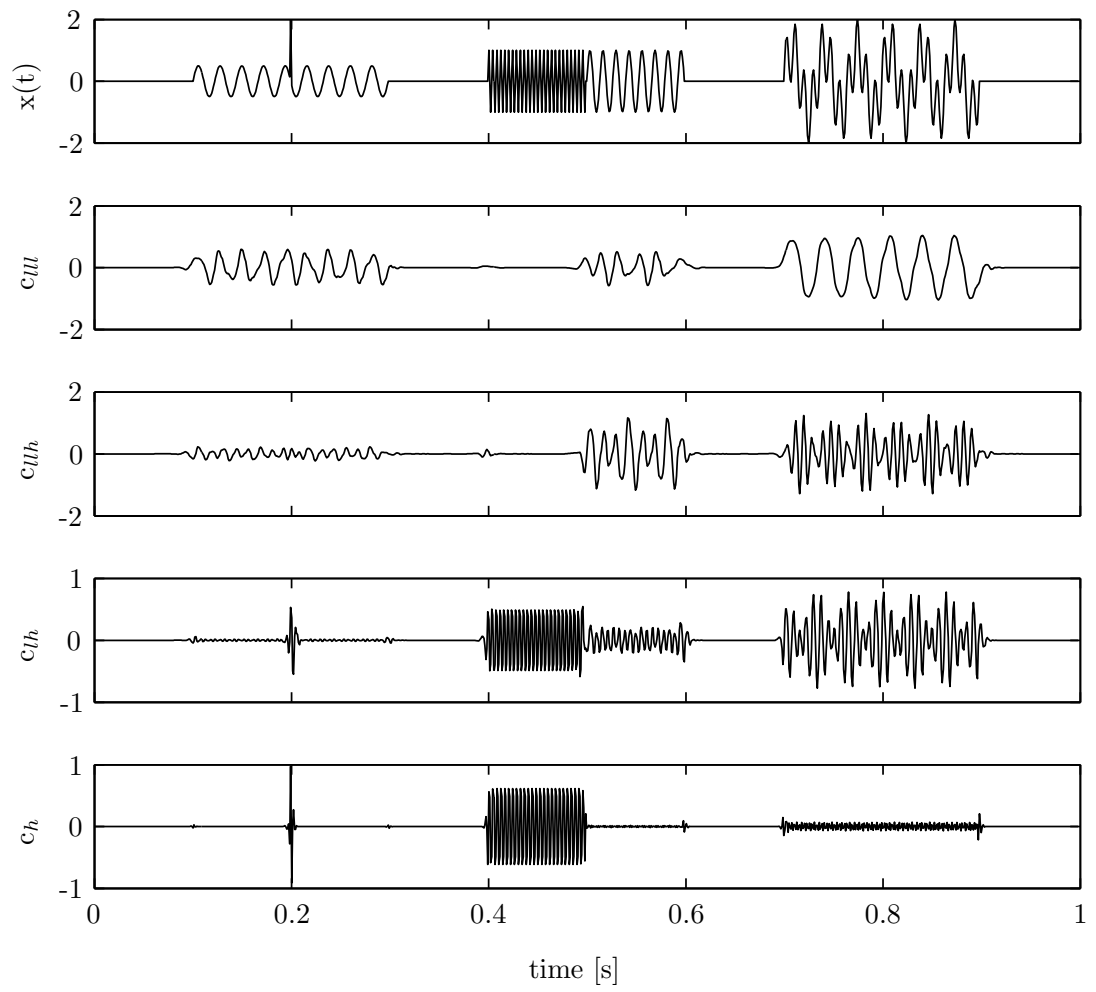

Figure 3.2: Discrete wavelet transform

The frequency content of the signal $x(t)$ of Fig. 3.2 is decomposed into the correct frequency bands. The sine with a frequency of $250 \mathrm{~Hz}$ is visible in both $c_{l h}$ and $c_{h}$ since this frequency 
is located exactly at the edge of both bands. Signal components also appear in surrounding subbands, however with lower amplitudes. This is because the low and high-pass filters are not perfect FIR filters. The peak at $0.2 \mathrm{~s}$ appears in the frequency bands with the highest frequencies.

\subsection{Wavelet filtering methods}

In order to remove the non-repetitive part of the error, a filtering method is designed based on the discrete wavelet transform. The general idea is that the repetitive part of the error leads to equal wavelet coefficients whereas the non-repetitive part results in different wavelet coefficients. The wavelet filtering methods distinguish between the repetitive and non-repetitive part. The wavelet coefficients are altered by calculating a similarity measure between sets of wavelet coefficients and thresholding. The adjusted wavelet coefficients are used in the synthesis filter bank of the DWT for the reconstruction of an error signal which ideally should contain only the repetitive part of the error.

The wavelet transform is preferred over the Fourier transform because of the preservation of time information. In the Fourier transform, only whole frequency bands can be changed, which alters the frequency content in the whole signal. The DWT decomposes the signal into frequency dependent coefficients from which the original signal can be reconstructed. The coefficients of the synthesis filter bank can be processed in several ways. By adjusting the wavelet coefficients, the local frequency content of the reconstructed signal can be changed compared to the original signal.

In literature, often thresholding of the wavelet coefficients is used for removing the unwanted noise of a signal. Two kinds of thresholding techniques are possible, soft and hard thresholding. On these thresholding techniques, several variations have been developed, e.g. [1, 12].

In general, thresholding of the wavelet coefficients is based on the assumption that the contribution of the disturbances is small compared to the error signal and thus leads to smaller wavelet coefficients. However as the iterations increase in ILC and the repetitive part of the error decreases, the disturbances start to dominate the error signals. Furthermore, especially for motion-systems, a small low-frequent input disturbance can be magnified to the same order of magnitude as the repetitive part of the error because of the large amplitude of the process sensitivity $S_{P}$ at low frequencies. Therefore, thresholding of the error signals directly is not considered to be a suitable method for removing the non-repetitive part of the error.

The removal of the non-repetitive part of the error is done based on three different wavelet filtering methods which all use the DWT. The wavelet function and decomposition level used for the DWT and the wavelet filtering method will be discussed in Section 3.2.1 and Section 3.2.2. The wavelet filtering methods will be presented in Section 3.2.3 up to Section 3.2.5.

\subsubsection{Wavelet function}

The succes of a certain wavelet decomposition depends strongly on the choice of the wavelet function [29]. Perfect reconstruction of the original signal with the wavelet coefficients of the DWT is only possible using the biorthogonal wavelet family [20]. However, since the wavelet coefficients are adjusted to remove the non-repetitive disturbances, this property is not required.

The optimal wavelet is chosen based on the maximum absolute error between a reference error signal $x_{\text {optimal }}$ and a reconstructed error signal $x_{\text {constr }}$ of the DWT

$$
e_{\text {wavelet }}=\max \left(\operatorname{abs}\left(x_{\text {optimal }}-x_{\text {constr }}\right)\right) .
$$

The wavelet function that results in the lowest error is used for the analysis and synthesis filter banks of the DWT. The used reference signals $x_{\text {optimal }}$ equal the error signals of the first and last iteration for an ILC simulation without additional disturbances. The reconstructed signals $x_{\text {constr }}$ are the reconstructed signals of the DWT with the designed wavelet filtering methods. The optimal wavelet function is the wavelet function for which the designed filtering methods remove the disturbances the best, resulting in a reconstructed error signal which resembles $x_{\text {optimal }}$ the most. 


\subsubsection{Decomposition level}

The decomposition level of the DWT determines the lowest frequency that can be observed in the DWT. The highest frequency which can be observed in the approximation coefficients of the DWT for a given decomposition level $p$ and sampling frequency $f_{s}$ equals

$$
f_{\max , c A}=\frac{f_{s}}{2^{p+1}}
$$

For the wavelet filtering methods, the decomposition level is chosen in such a way that the approximation coefficients contain most part of the error of the first iteration. The desired maximum frequency of the approximation coefficients $f_{\text {max,approx }}$ is determined using a FFT of the error of the first iteration of ILC. The decomposition level of the DWT can be calculated as

$$
p=\text { floor }\left(\frac{\log \left(f_{s} / f_{\max , \text { approx }}\right)}{\log (2)}-1\right)
$$

\subsubsection{Two run method}

In order to distinguish between the repetitive and non-repetitive part of the error, at least two error signals have to be available. These error signals are obtained by performing two runs at a single iteration of ILC. A run is defined as a simulation or experiment, unrelated to wether a feedforward update is performed. The number of iterations is defined as the number of feedforward updates in ILC.

The DWT decomposes the error signal of both runs into two sets of wavelet coefficients at all frequency bands. These wavelet coefficients contain an equal repetitive part $c_{r}$ and different non-repetitive parts $c_{n r}$ as

$$
\begin{aligned}
& c_{1}=c_{r}+c_{n r, 1} \\
& c_{2}=c_{r}+c_{n r, 2} .
\end{aligned}
$$

Note that the subscripts here denote the number of the run, rather than the iteration number. The difference between the wavelet coefficients $\Delta c$ contains only the non-repetitive parts. The mean of the wavelet coefficients $\bar{c}$ contains the repetitive part and an average non-repetitive part

$$
\begin{aligned}
\Delta c & =c_{1}-c_{2}=c_{n r, 1}-c_{n r, 2} \\
\bar{c} & =\frac{c_{1}+c_{2}}{2}=c_{r}+\frac{c_{n r, 1}+c_{n r, 2}}{2} .
\end{aligned}
$$

Since thresholding of the wavelet coefficients of the error signal directly is not preferred, the nonrepetitive part of the error has to be identified otherwise. For this a similarity measure is designed. The similarity between the wavelet coefficients is determined by dividing the difference $\Delta c$ by the mean $\bar{c}$, i.e. the relative difference between the two sets of wavelet coefficients

$$
S I M_{c_{1}, c_{2}}=\frac{\Delta c}{\bar{c}}
$$

Wavelet coefficients that have a similarity criterion smaller than a predefined level $\gamma$ are maintained during the synthesis of the DWT. Wavelet coefficients with a similarity criterion larger than $\gamma$ are removed from the synthesis filter bank, i.e. they are set to zero. This leads to an adjusted set of wavelet coefficients:

$$
c_{a d j}= \begin{cases}\bar{c}, & \text { if }\left|S I M_{c_{1}, c_{2}}\right|<\gamma \\ 0, & \text { if }\left|S I M_{c_{1}, c_{2}}\right| \geq \gamma\end{cases}
$$

The threshold level $\gamma$ consists of two parts, a constant level $\gamma_{c}$ and a variable part $\gamma_{v a r}$

$$
\gamma=\gamma_{c} \gamma_{v a r}
$$


The constant part $\gamma_{c}$ can be chosen based on the allowed difference between the wavelet coefficients. In other words, the amount which the coefficients of the repetitive part may vary. The variable part is adjusted based on the mean power, i.e. the squared root-mean-square (rms) values of $\Delta c$ and $\bar{c}$. The variable part of the threshold is defined as

$$
\gamma_{v a r}=\frac{\operatorname{rms}(\bar{c})^{2}}{\operatorname{rms}(\Delta c)^{2}}
$$

If the wavelet coefficients are dominated by the non-repetitive part $(\operatorname{large} \operatorname{rms}(\Delta c))$, the threshold level is lowered and vice versa. The rms value of a discrete signal $x$ of length $n$ is calculated as

$$
\operatorname{rms}(x)=\sqrt{\frac{1}{n} \sum_{i=1}^{n} x_{i}^{2}}
$$

The coefficient adjustment method, described above, is performed at all frequency bands. The threshold level $\gamma$ is altered for each separate frequency band, based on the mean power of the wavelet coefficients in the various frequency bands.

\subsubsection{Recursive method}

A disadvantage of the two run method is that at each iteration, two runs are needed. This doubles the number of runs that have to be performed compared to classic ILC. Since the adjustment of the repetitive part of the error is known, a recursive formulation can be made. With the recursive formulation, a feedforward update can be done after each run.

The learned feedforward affects the tracking error through the process sensitivity $S_{P}$. The reconstructed error $e_{k}^{*}$ of an iteration $k$ can be obtained from the error at iteration $k-1$ and the feedforward update as

$$
e_{k}^{*}=e_{k-1}-S_{P}\left(f_{k}-f_{k-1}\right)
$$

Again, two error signals are present at each iteration, the measured error $e_{k}$ and the reconstructed error signal $e_{k}^{*}$. On these two error signals, a DWT can be performed, leading to two sets of wavelet coefficients. Now a similarity criterion can be calculated and a coefficients adjustment can be performed analogues to the two run method.

Note that in the first iteration $(k=1)$, no reconstructed error signal $e_{1}^{*}$ can be calculated. To prevent the disturbances of the first iteration from entering the learning scheme and the learned feedforward, still two runs have to be performed at the first iteration.

\subsubsection{Higher order method}

If more runs are performed at equal iterations, more signals are available which all have an equal repetitive part and different non-repetitive parts. Therefore, it is easier to distinguish between the repetitive and non-repetitive part of the error. A drawback is that the number of runs needed compared to classic ILC increases even more than for the two run method.

In this section, a wavelet filtering method is discussed which uses four runs at each iteration. This leads to four different error signals of which a DWT is performed. In each frequency band of the DWT, four different sets of wavelet coefficients are present with an equal repetitive part $c_{r}$ and different non-repetitive parts $c_{n r}$

$$
\begin{aligned}
& c_{1}=c_{r}+c_{n r, 1} \\
& c_{2}=c_{r}+c_{n r, 2} \\
& c_{3}=c_{r}+c_{n r, 3} \\
& c_{4}=c_{r}+c_{n r, 4} .
\end{aligned}
$$


With the four sets of wavelet coefficients, two difference variables $\left(d_{1}, d_{2}\right)$ and mean wavelet coefficients variables $\left(m_{1}, m_{2}\right)$ can be calculated analogues to the two run method as

$$
\begin{aligned}
d_{1} & =c_{1}-c_{2}=c_{n r, 1}-c_{n r, 2} \\
d_{2} & =c_{3}-c_{4}=c_{n r, 3}-c_{n r, 4} \\
m_{1} & =\frac{c_{1}+c_{2}}{2}=c_{r}+\frac{c_{n r, 1}+c_{n r, 2}}{2} \\
m_{2} & =\frac{c_{3}+c_{4}}{2}=c_{r}+\frac{c_{n r, 3}+c_{n r, 4}}{2} .
\end{aligned}
$$

Using these equations, two similarity criteria are defined as

$$
\begin{gathered}
S_{1}=\frac{d_{1}}{m_{1}} \\
S_{2}=\frac{d_{2}}{m_{2}} .
\end{gathered}
$$

For the synthesis of the DWT, the wavelet coefficients for which both similarity criteria are smaller than the threshold levels $\gamma_{1}, \gamma_{2}$ are kept

$$
c_{a d j}=\left\{\begin{array}{cl}
\frac{c_{1}+c_{2}+c_{3}+c_{4}}{4}, & \text { if }\left|S_{1}\right|<\gamma_{1} \text { and }\left|S_{2}\right|<\gamma_{2} \\
0, & \text { if }\left|S_{1}\right| \geq \gamma_{1} \text { or }\left|S_{2}\right| \geq \gamma_{2} .
\end{array}\right.
$$

The adjustment method uses for both similarity criteria different thresholds $\gamma_{1}, \gamma_{2}$. Both consist of an equal constant part $\gamma_{c}$ and a different variable part $\gamma_{v a r_{1,2}}$ which changes based on the mean power of the signals in the corresponding similarity criterion. The variable parts are defined as

$$
\begin{aligned}
\gamma_{v a r, 1} & =\frac{\operatorname{rms}\left(m_{1}\right)^{2}}{\operatorname{rms}\left(d_{1}\right)^{2}} \\
\gamma_{v a r, 2} & =\frac{\operatorname{rms}\left(m_{2}\right)^{2}}{\operatorname{rms}\left(d_{2}\right)^{2}} .
\end{aligned}
$$

This coefficient adjustment method is also applied to all frequency bands of the DWT. More variations of the similarity criteria on can be thought of using the four sets of wavelet coefficients, however we restrict ourselves to the one above.

\subsubsection{Additional remarks}

All wavelet filtering methods use error signals which are decomposed into wavelet coefficients at different frequency subbands. The various sets of wavelet coefficients are adjusted in order to remove the non-repetitive part. The adjustment is performed at all subbands separately. The adjusted wavelet coefficients can be used in the synthesis filter bank of the DWT in order to construct an error signal which should ideally contain only the repetitive part of the error. With the designed filtering methods, the disturbances do not enter the learning scheme and do not affect the learned feedforward signal.

If the non-repetitive disturbances in a frequency band have an intersection, this will lead to equal wavelet coefficients at that time instant. The wavelet coefficients of that time instant and frequency band are passed by the similarity criterion adjustment and they appear in the adjusted wavelet coefficients. This results in peaks in the reconstructed wavelet coefficients. Therefore, single coefficients are removed from the adjusted coefficients by checking whether the two surrounding wavelet coefficients are also included in the reconstruction.

If the disturbances have an equal magnitude and opposite sign, they are passed by the similarity criterion since $\Delta c$ equals zero. However, because the mean is taken in the adjusted wavelet coefficients, the disturbances cancel each other and still a disturbance free error signal is obtained. A schematic representation of the control scheme with wavelet filtering and ILC is shown in Fig. 3.3. 
The wavelet filtering methods can be applied in the following steps

1. Decompose the error signals into sets of wavelet coefficients at various frequency bands using the DWT.

2. Calculate for all frequency bands the similarity measure(s) and the threshold level(s).

3. Adjust the wavelet coefficients of the various frequency bands.

4. Perform a reconstruction with the adjusted wavelet coefficients in a synthesis filter bank to reconstruct a disturbance free error signal.

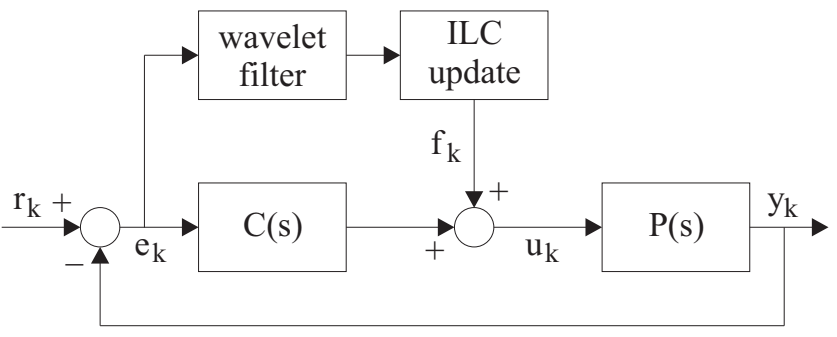

Figure 3.3: ILC scheme with wavelet filtering

The reconstructed error signal is used as input for the learning scheme of ILC. With the wavelet filtering the disturbances are removed from the tracking error, leading to an improved performance by a decrease in the error after convergence and a disturbance free feedforward signal.

\subsection{Discussion}

The non-repetitive part of the error can be removed by filtering the error signal before it enters the learning scheme of ILC. The presented filtering methods are based on the discrete wavelet transform (DWT).

The presented wavelet filtering methods remove the non-repetitive part of the error. However, some part of the non-repetitive errors can remain in the reconstructed error signal if the wavelet coefficients of the non-repetitive errors are below the threshold. Contrarily, some of the repetitive errors can also be removed together with the non-repetitive errors. This occurs at the frequencies and time instants where the non-repetitive errors dominate the repetitive part.

The DWT decomposes the error signals into wavelet coefficients at various frequency bands. The non-repetitive part is identified in the designed methods by calculation of a similarity measure between two sets of wavelet coefficients. The identification of the non-repetitive part of the error can perhaps also be done by adjusting the wavelet coefficients in other ways, e.g. by using different similarity measures.

The threshold level $\gamma$ consists of a constant part $\gamma_{c}$ and a varying part $\gamma_{v a r}$. The constant part of the threshold has to be tuned manually. The performance of the filtering methods is also determined by the choice of $\gamma_{c}$. However, it is not very difficult to tune $\gamma_{c}$. If $\gamma_{c}$ is chosen too small, repetitive parts of the error are also removed, leading to an increase in the tracking error of ILC after convergence. If $\gamma_{c}$ is chosen too high, disturbances will remain in the reconstructed error signals and the tracking error will also increase.

The higher order method performs four runs at each iteration of ILC. This results in four sets of wavelet coefficients. Using these four sets two similarity criteria are designed. The higher order method can be exploited further, i.e. more criteria can be calculated using the four sets of wavelet coefficients. In this thesis we restrict ourselves to the version with two similarity criteria. 


\section{Chapter 4}

\section{Simulations}

The disturbance analysis of Chapter 2 and the designed wavelet filtering methods of Chapter 3 will be validated and tested in this chapter by means of simulations. In the simulations the nonrepetitive disturbances are known since they are added separately. This makes it easier to interpret the results of the disturbance analysis validation and determine the quality of the designed wavelet filtering methods.

The simulations will be performed on a fourth order system. First the classic ILC concept will be applied in simulations in Section 4.2. The disturbance analysis of Chapter 2 will be validated in Section 4.3. The most simple method for eliminating disturbances is by averaging, which will be tested in Section 4.4. Simulations with the wavelet filtering methods of Chapter 3 will be performed in Section 4.5. All simulations will be performed at a fixed sampling rate $f_{s}$ of $8 \mathrm{kHz}$.

\subsection{Simulation model}

A schematic representation of the fourth order system is shown in Fig. 4.1. The system consists of two masses that are connected by a spring and a damper. The force exerted on the first mass is denoted by $F$, the position of the second mass is denoted by $x$. The simulation model resembles the system which will be used in the experiments of Chapter 5, which consists of two rotating masses which are connected through a flexible shaft.

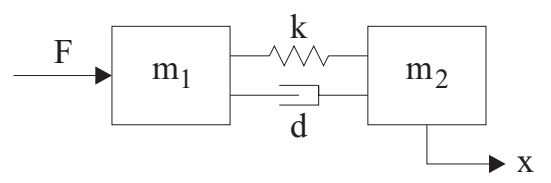

Figure 4.1: schematic representation

The equations of motion can be written as

$$
\begin{aligned}
& m_{1} \ddot{x}_{1}=F-k\left(x_{1}-x_{2}\right)-d\left(\dot{x}_{1}-\dot{x}_{2}\right) \\
& m_{2} \ddot{x}_{2}=k\left(x_{1}-x_{2}\right)+d\left(\dot{x}_{1}-\dot{x}_{2}\right) .
\end{aligned}
$$

The transfer function from the force $F$ to the position $x$ can be written with use of Laplace as

$$
\frac{X}{F}=\frac{d s+k}{s^{2}\left(m_{1} m_{2} s^{2}+d s\left(m_{1}+m_{2}\right)+k\left(m_{1}+m_{2}\right)\right)} .
$$


The behavior of the transfer function for $s \rightarrow 0$ and $s \rightarrow \infty$ equals

$$
\begin{aligned}
s \rightarrow 0: & \frac{X}{F}=\frac{k}{s^{2} k\left(m_{1}+m_{2}\right)} \\
s \rightarrow \infty: & \frac{X}{F}=\frac{d s}{m_{1} m_{2} s^{4}} .
\end{aligned}
$$

For $s \rightarrow 0$ the transfer function has a slope of -2 and a phase of $180^{\circ}$, for $s \rightarrow \infty$ the transfer function has a slope -3 and a phase of $-270^{\circ}$. If no damping is present in the system, the transfer function has for $s \rightarrow \infty$ a slope -4 and a phase of $-360^{\circ}$ according to

$$
s \rightarrow \infty: \quad \frac{X_{2}}{F}=\frac{k}{m_{1} m_{2} s^{4}} .
$$

\subsection{Classic ILC}

The frequency response function (FRF) from the force $F$ to the position $x$ of the second mass is shown in Fig. 4.2. Using this FRF, a feedback controller is designed which results in a bandwidth of the closed loop system of $5 \mathrm{~Hz}$.

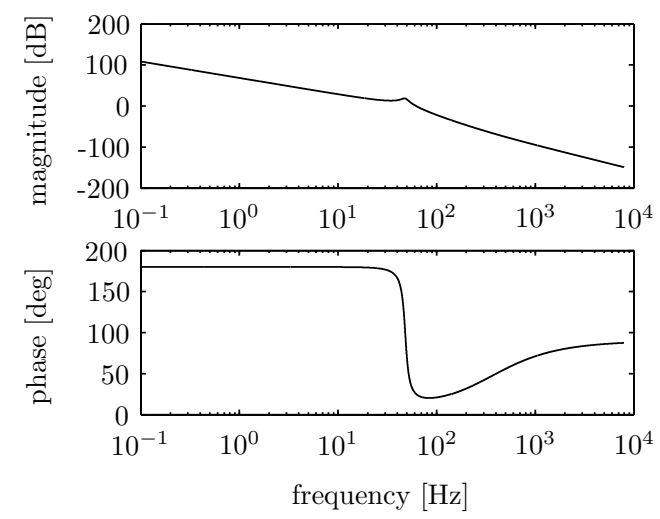

Figure 4.2: FRF system
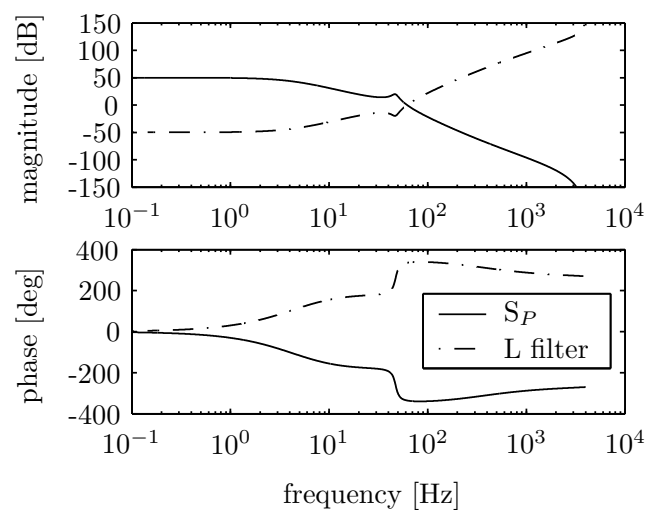

Figure 4.3: FRFs $S_{P}$ and $L$ filter

The learning filter $L$ of Fig. 4.3 is designed using the zero phase error tracking (ZPETC) method [36]. The ZPETC algorithm calculates an alternative for the exact inverse $S_{P}$. The algorithm assumes that a discrete model of the $S_{P}$ and a closed loop stabilizing controller are available. The ZPETC method calculates an inverse with zero phase error, however an amplitude error is introduced. The amplitude distortion is usually located at high frequencies because this is where the unstable zeros are often located. The non-causality of the $L$ filter is eliminated by shifting the filtered signal back in time, which can be done since the feedforward signal is calculated off-line. A more detailed description of the ZPETC algorithm can be found in Appendix A.

In order to design the learning filter $L$, the process sensitivity $S_{P}$ is calculated using the system model and the designed controller. Of the obtained transfer function of $S_{P}$, a minimal realization (pole-zero cancelation) is obtained using the routine minreal.m in the control toolbox of Matlab Version 6.5. The resulting transfer function is discretized using the routine c2dm.m with a Tustin discretization method. A stable approximation of the inverse $S_{P}$ is obtained using zpetc.m [36, 8]. The use of this routine introduces some samples delay $(p h d)$. The additional phase delay can be compensated for by shifting the time signals back over the number of samples delay. In the frequency domain the $L$ filter with phase correction $L_{c}$ can be calculated using

$$
\angle\left(L_{c}(\omega)\right)=\angle\left(L(\omega)+\omega p h d t_{s}\right),
$$




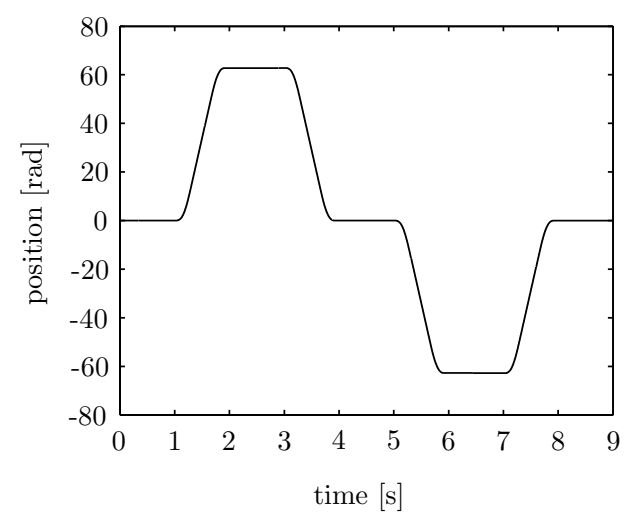

Figure 4.4: Reference signal

where the frequency in $\mathrm{rad} / \mathrm{s}$ is denoted by $\omega$ and the sample time by $t_{s}$. For the simulations a second order low-pass $Q$ filter with a cut-off frequency $f_{c}$ of $1000 \mathrm{~Hz}$ is used. The reference signal to be tracked is shown in Fig. 4.4.

The performance of ILC without additional disturbances is tested in a simulation with 25 iterations. For the first iteration, a zero feedforward signal is used. The maximum absolute tracking errors of the 25 iterations are shown in Fig. 4.5(a). The tracking error converges in five iterations to $|e|_{\max }=1.15 \cdot 10^{-10} \mathrm{rad}$. The learned feedforward of the $25^{\text {th }}$ iteration is shown in Fig. 4.5(b).

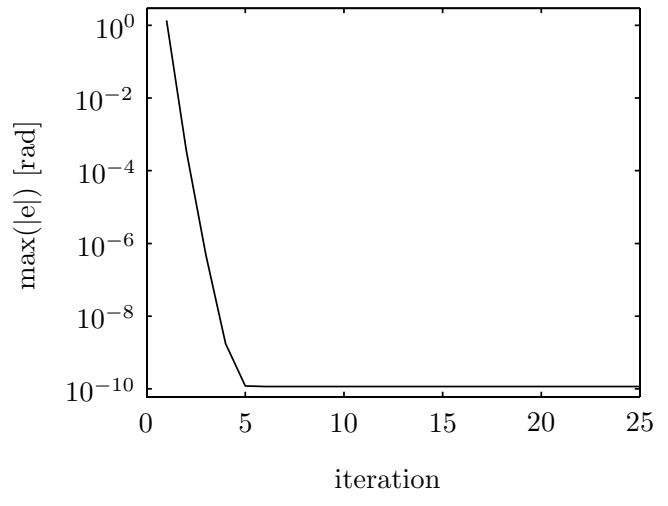

(a) Maximum absolute error

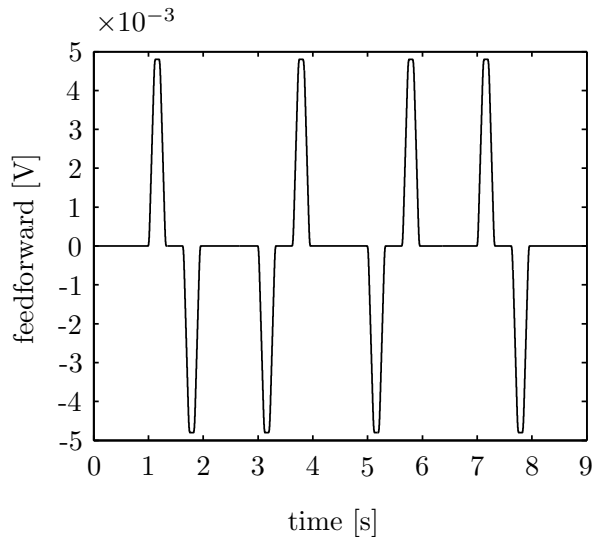

(b) Feedforward signal

Figure 4.5: Results without disturbances

\subsection{Disturbance analysis}

The expression for the tracking error (2.10) of Section 2.2 will be validated by means of simulations in this section. The influence of measurement disturbances, load disturbances and model uncertainties will be validated separately. The influence of disturbances will be analyzed using both harmonic disturbances and stochastic disturbances. The influence of model uncertainties will be investigated for model gain and model order uncertainties. 


\subsubsection{Measurement disturbances}

The influence of measurement disturbances $d_{k}$ on the tracking error of ILC follows from $(2.10)$ as

$$
e_{k, d}=-S d_{k}+S_{P} Q L S d_{k-1}+\sum_{j=1}^{k-2} S_{P} Q\left[Q\left(1-L S_{P}\right)\right]^{k-j-1} L S d_{j} .
$$

The harmonic measurement disturbance is chosen such that the amplitude exceeds the magnitude of the tracking error of the ILC experiment without additional disturbance (see Fig. 4.5(a)). The frequency is chosen below the cut-off frequency of the $Q$ filter. The initial phase $\phi_{0}$ varies randomly every iteration. The used harmonic measurement disturbance $d_{k}$ equals

$$
d_{k}=1 \cdot 10^{-2} \sin \left(2 \pi 100 t+\phi_{0}\right) .
$$

\section{Two iterations}

From the disturbance analysis of Section 2.2 followed that for an ideal learning filter, i.e. $L=S_{P}^{-1}$, the disturbances of the last two iterations determine the tracking error. In the worst case, the disturbance signals of the last two iterations have opposite sign, which for harmonic measurement disturbances corresponds to an initial phase shift of $\pi \mathrm{rad}$.

The error signal of the second iteration contains the disturbance signal of the second iteration filtered by the sensitivity $S$ and the disturbance signal of the first iteration filtered by $S_{P} Q L S$. The magnitudes of the FRFs of $S$ and $S_{P} Q L S$ are shown in Fig. 4.6. At the disturbance frequency

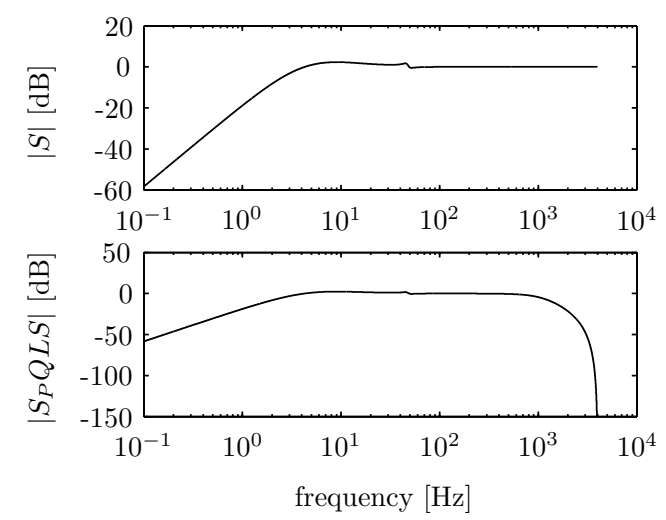

Figure 4.6: Magnitudes FRFs $S$ and $S_{P} Q L S$

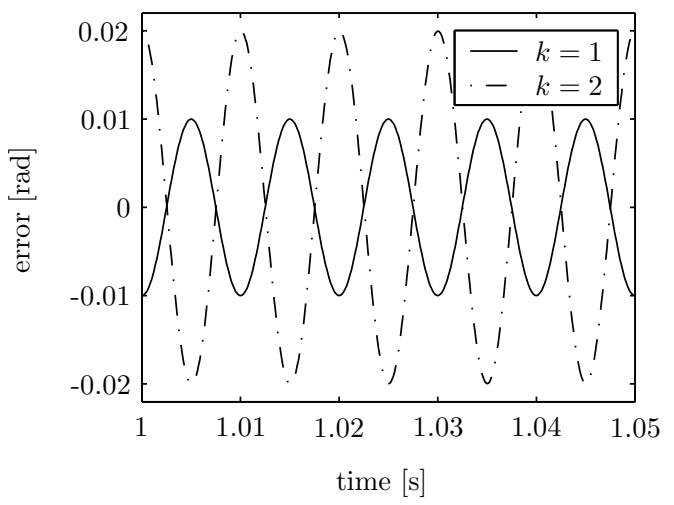

Figure 4.7: Errors worst case simulation

of $100 \mathrm{~Hz}$ the magnitudes of $S$ and $S_{P} Q L S$ equal

$$
\begin{aligned}
|S| & =0.9980 \\
\left|S_{P} Q L S\right| & =0.9964 .
\end{aligned}
$$

The maximum tracking errors of the first and second iterations can be calculated using (4.8) as

$$
\begin{aligned}
& \left|e_{1, d}\right|_{\max }=|S| d_{\max }=0.9980 \cdot 10^{-2} \mathrm{rad} \\
& \left|e_{2, d}\right|_{\max }=|S| d_{\max }+\left|S_{P} Q L S\right| d_{\max }=1.9943 \cdot 10^{-2} \mathrm{rad}
\end{aligned}
$$

In order to evaluate the effect of the disturbances only, the initial feedforward equals the feedforward signal obtained from the simulation without disturbances of Fig. 4.5(b). With this initial feedforward, the error caused by the reference $r$ is much smaller than the errors caused by the measurement disturbances.

The tracking errors of the first and second iteration for the worst case simulation with two iterations are shown in Fig. 4.7. The amplification of the tracking error is caused by the presence of the disturbance signals in the learning process of the feedforward signal. 
Table 4.1: Maximum absolute errors with measurement disturbances

\begin{tabular}{c|c|c|c}
\multirow{2}{*}{ method } & \multicolumn{3}{|c}{ maximum absolute error [rad] } \\
\cline { 2 - 4 } & first iteration & second iteration & 25 iterations \\
\hline calculated & $0.9980 \cdot 10^{-2}$ & $1.9943 \cdot 10^{-2}$ & - \\
simulation & $0.9979 \cdot 10^{-2}$ & $1.9954 \cdot 10^{-2}$ & $2.0284 \cdot 10^{-2}$
\end{tabular}

The calculated maximum absolute errors and the maximum absolute errors obtained from the simulation are given in Table 4.1. The calculated maximum errors show a good correspondence with the values found in the simulation.

\section{Multiple iterations}

For an ILC simulation with more than two iterations, the measurement disturbances of older iterations affect the tracking error through the summation term in (4.8). From the disturbance analysis of Section 2.2 followed that the disturbances of iterations older than the last two are not likely to influence the maximum error significantly.

Fig. 4.8 shows the maximum absolute error of an ILC simulation with 25 iterations and random initial phases of the disturbance signals. If the disturbance signals of the last two iterations have a very small phase difference, the disturbances become almost repetitive over the two iterations and the resulting error can become temporarily smaller than $|S| d_{\max }$.

The largest maximum error after convergence is given in Table 4.1. The largest simulated maximum absolute error exceeds the calculated worst case error with two iterations only by $1.7 \%$ and the simulated worst case error only by $1.6 \%$. The contribution of the summation term of (4.8) is much smaller than the contribution of the other two terms, as expected.

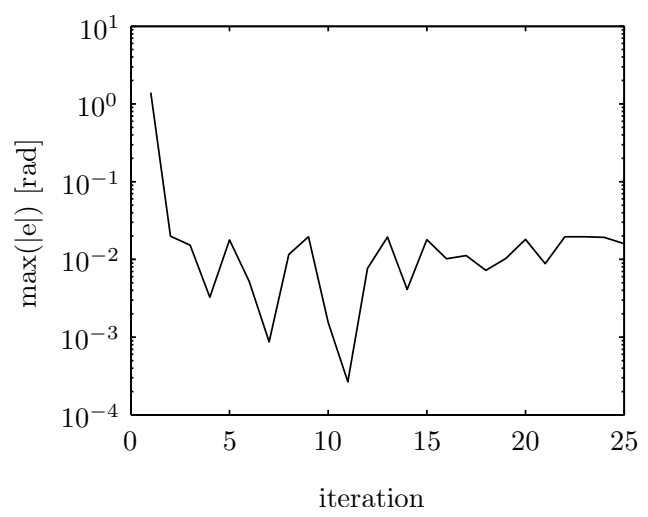

Figure 4.8: Maximum absolute error with measurement disturbances

\subsubsection{Load disturbances}

The load disturbances affect the tracking error according to (2.10) as

$$
e_{k, n}=-S_{P} n_{k}+S_{P}^{2} Q L n_{k-1}+\sum_{j=1}^{k-2} S_{P}^{2} Q\left[Q\left(1-L S_{P}\right)\right]^{k-j-1} L n_{j}
$$


Table 4.2: Maximum absolute errors with load disturbances

\begin{tabular}{c|c|c|c}
\multirow{2}{*}{ method } & \multicolumn{3}{|c}{ maximum absolute error [rad] } \\
\cline { 2 - 4 } & first iteration & second iteration & 25 iterations \\
\hline calculated & 2.9877 & 5.8855 & - \\
simulation & 2.9878 & 5.9757 & 5.9992
\end{tabular}

The used harmonic load disturbance $n_{k}$ has an amplitude exceeding the maximum error obtained without additional disturbances and a frequency below the cut-off frequency of the $Q$ filter

$$
n_{k}=1 \cdot 10^{-2} \sin \left(2 \pi 1 t+\phi_{0}\right) \text {. }
$$

\section{Two iterations}

The effect of the load disturbances of the first two iterations on the error $e_{k}$ is given by (4.10). The error of the second iteration contains the disturbance signal of the second iteration filtered by the process sensitivity $S_{P}$ and the disturbance signal of the first iteration filtered by $S_{P}^{2} Q L$. Fig. 4.9 shows the magnitudes of the FRFs of $S_{P}$ and $S_{P}^{2} Q L$.

At the disturbance frequency of $1 \mathrm{~Hz}$ the magnitudes of $S_{P}$ and $S_{P}^{2} Q L$ equal

$$
\begin{aligned}
\left|S_{P}\right| & =298.7727 \\
\left|S_{P}^{2} Q L\right| & =289.7752 .
\end{aligned}
$$

The maximum absolute errors of the first and second iterations can be calculated using (4.10) as

$$
\begin{aligned}
& \left|e_{1, n}\right|_{\max }=\left|S_{P}\right| n_{\max }=2.9877 \mathrm{rad} \\
& \left|e_{2, n}\right|_{\max }=\left|S_{P}\right| n_{\max }+\left|S_{P}^{2} Q L\right| n_{\max }=5.8855 \mathrm{rad} .
\end{aligned}
$$

As was the case for the measurement disturbances, the worst case occurs when the harmonic disturbances of the last two iterations have opposite signs, i.e. an initial phase shift of $\pi \mathrm{rad}$.

The tracking errors of the two iterations of the worst case simulation with load disturbances are shown in Fig. 4.10. The initial feedforward signal equals the feedforward signal of Fig. 4.5, obtained from the simulation without disturbances.
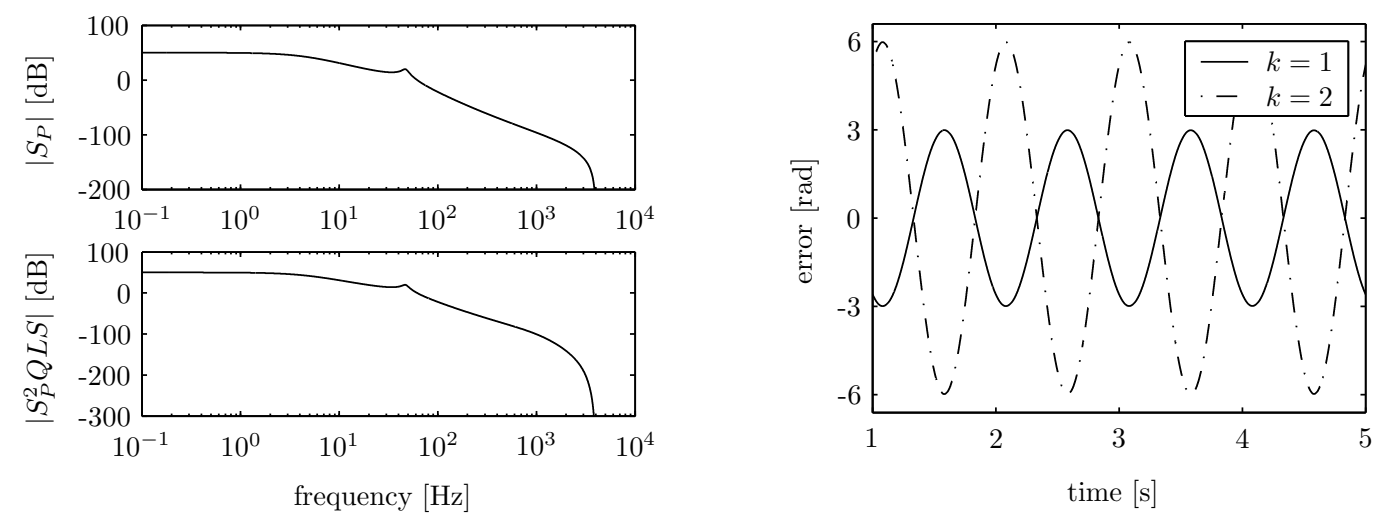

Figure 4.9: Magnitudes FRFs $S_{P}$ and $S_{P}^{2} Q L$

Figure 4.10: Errors worst case simulation

The maximum absolute errors of the two iterations are given in Table 4.2. The calculated values correspond to the values obtained in the simulation. 


\section{Multiple iterations}

For multiple iterations, the load disturbances of the iterations older than the last two are filtered by the summation term in (4.10). The disturbance signals of iterations older than the last two are not likely to have a large influence on the tracking error. This also follows from the ILC simulation with 25 iterations and a random initial phase of the disturbance signal, as can be seen in Fig. 4.11. The maximum absolute error shows no convergence, which is because the error due to the disturbances is of the same order of magnitude as the error of the first iteration.

Table 4.2 gives the largest maximum absolute error after convergence of the simulation with 25 iterations. The maximum error obtained from the 25 iterations exceeds the worst case values of 2 iterations only a bit, indicating the limited contribution of the summation term.

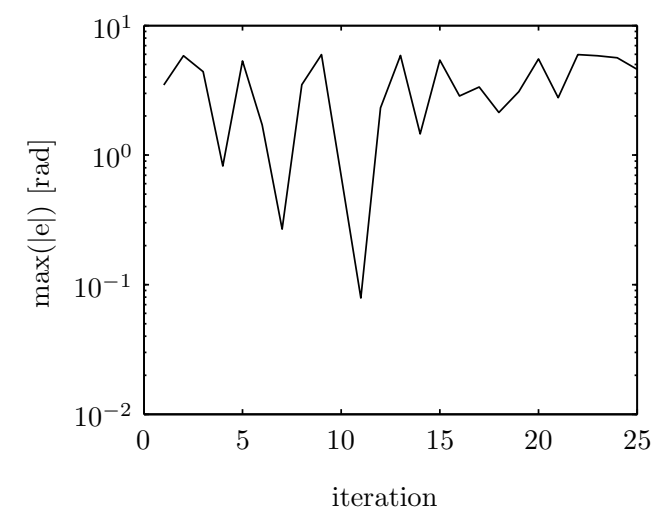

Figure 4.11: Maximum absolute error with load disturbances

\subsubsection{Stochastic disturbances}

In practice, the non-repetitive errors are often stochastic signals, e.g. noise. The validity of (2.10) for stochastic disturbances is tested by a simulation with 2 iterations and noise measurement disturbances. The noise signals have a uniform distribution in the interval $\left[-1 \cdot 10^{-2}, 1 \cdot 10^{-2}\right] \mathrm{rad}$. The initial feedforward equals the feedforward obtained from the simulation without additional disturbances as shown in Fig. 4.5(b). The maximum absolute error and root-mean-square (rms) values of the error signals of the two iterations are given in Table 4.3. Table 4.3 also contains the calculated values. The calculated values using (4.8) and the values obtained by the simulation are nearly equal, indicating the validity of (2.10) for stochastic disturbances.

Table 4.3: Maximum absolute values and rms values of the error signals for noise disturbances

\begin{tabular}{c|c|c|c} 
iteration & value & calculated & simulation \\
\hline 1 & $\max \left(\left|e_{1}\right|\right)$ & $1.1268 \cdot 10^{-2} \mathrm{rad}$ & $1.1269 \cdot 10^{-2} \mathrm{rad}$ \\
1 & $\operatorname{rms}\left(e_{1}\right)$ & $5.7790 \cdot 10^{-3} \mathrm{rad}$ & $5.7784 \cdot 10^{-3} \mathrm{rad}$ \\
\hline 2 & $\max \left(\left|e_{2}\right|\right)$ & $1.7992 \cdot 10^{-2} \mathrm{rad}$ & $1.8315 \cdot 10^{-2} \mathrm{rad}$ \\
2 & $\operatorname{rms}\left(e_{2}\right)$ & $6.3825 \cdot 10^{-3} \mathrm{rad}$ & $6.3251 \cdot 10^{-3} \mathrm{rad}$
\end{tabular}

\subsubsection{Model uncertainties}

The influence of model uncertainties on the tracking error has already been discussed in Section 2.2.3. The validation will be performed in this section by means of simulations for model gain 
uncertainties with and without disturbances and for model order uncertainties without additional disturbances. For all model uncertainties $\Delta P$, the same feedback controller $C$ is used in order to evaluate only the effect of the various model uncertainties on the tracking error.

\section{Model gain uncertainty}

The model gain uncertainty is modeled as an additive uncertainty in (2.12). Simulations are performed for both a negative model uncertainty $\Delta P=-0.1 P$ and a positive model uncertainty $\Delta P=+0.1 P$. The model uncertainties influence the learning filters $\tilde{L}$.

For the case of no disturbances the tracking error is only determined by the reference $r$. The reference signal $r$ contains mainly frequencies below the bandwidth. The FRFs of $S_{P} Q \tilde{L} S$ with a negative and positive $\Delta P$ have below the bandwidth respectively a smaller and larger magnitude than the nominal system, as can be seen in Fig. 4.12.

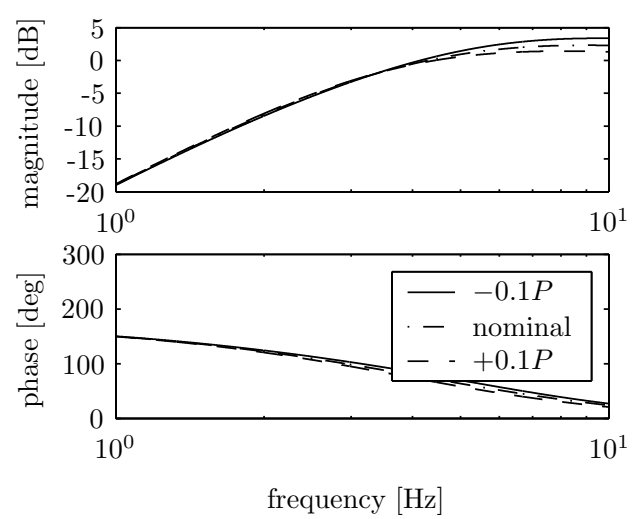

Figure 4.12: FRFs $S_{P} Q \tilde{L} S$

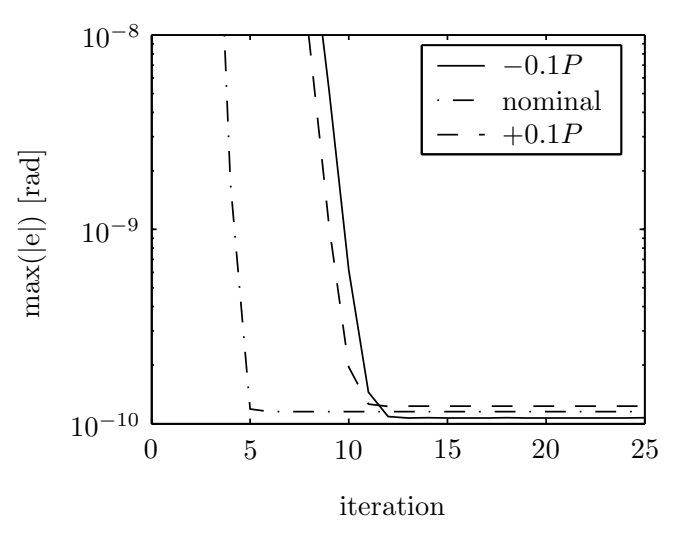

Figure 4.13: Errors with gain uncertainties

The maximum absolute errors of the simulations with 25 iterations and the various model uncertainties are shown in Fig. 4.13. The simulations with a positive $\Delta P$ lead to a larger maximum absolute tracking error after convergence in comparison to the nominal fit. With a negative $\Delta P$, a smaller tracking error than the nominal fit is obtained. This corresponds to the results of [10] which specifies uncertainties on the process sensitivity $S_{P}$. Furthermore it can be seen that the presence of model uncertainties leads to a slower convergence rate.

The model uncertainties affect also the way measurement and load disturbances influence the tracking error. The learning filters with model uncertainty $\tilde{L}$ change the amplitudes of the disturbance filters of the various iterations (see (3.1)). The validation of the way model uncertainties influence the effect of disturbances on the tracking error is described in [22] (see Appendix D).

\section{Model order uncertainty}

In practice, the model of the process sensitivity $S_{P}$, used for the design of the learning filter $L$, forms an approximation of the real system behavior. Resonances at high frequencies are often ignored. The FRFs of the fourth order system and a model without resonance are shown in Fig. 4.14. The simulation with the simplified model has the largest errors at frequencies around the resonance frequency. At these frequencies, the simplified model corresponds in this case to a frequency dependent negative model gain uncertainty.

In order to satisfy the convergence criterion of (2.4), a fourth order low-pass robustness filter $Q$ with a cut-off frequency of $28 \mathrm{~Hz}$ is needed. The maximum absolute tracking errors of the simulations performed with learning filters $\tilde{L}$, designed with the nominal system and the simplified model are shown in Fig. 4.15. The change in $Q$ filter results in an increase in tracking error from order of magnitude $10^{-10}$ to $10^{-5}$, as can be seen in Fig. 4.5(a) and Fig. 4.15. With the simplified model, a smaller error after convergence and a somewhat decreased convergence rate is obtained, which was also the case for the negative model gain uncertainty of Fig. 4.13. 

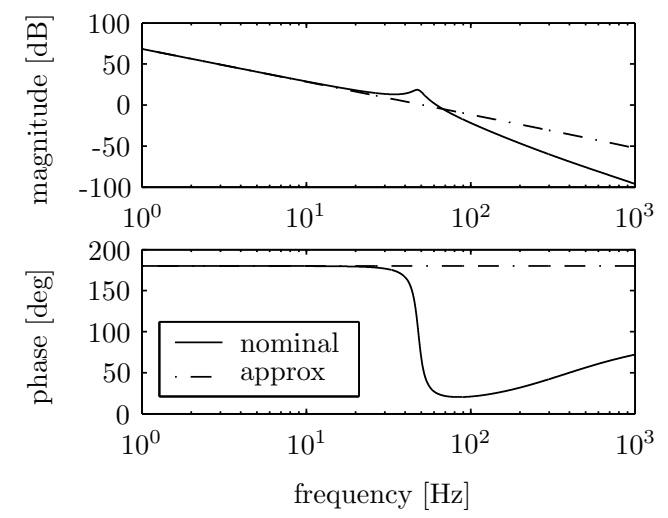

Figure 4.14: FRFs system and simplified model

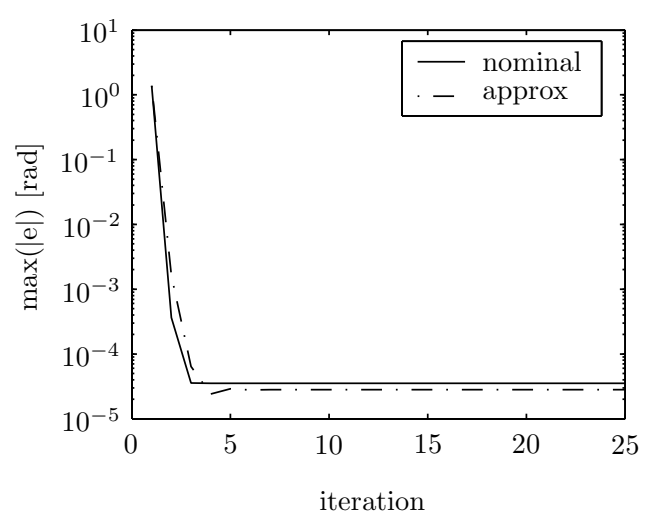

Figure 4.15: Errors with order uncertainty

\subsection{Averaging}

The most simple method to eliminate the repetitive part of the error signal of ILC is by performing several runs at each iteration of ILC and calculating the average. In this section, this approach is tested by means of simulations for non-repetitive deterministic disturbances and ten runs at each iteration of ILC. Using the ten tracking errors, an average tracking error is calculated which is used to update the feedforward signal. This approach is tested for both measurement and load disturbances. The disturbances have constant frequencies and amplitudes and varying initial phases $\phi_{0}$, as described in Section 4.3.

The maximum absolute tracking errors of the simulation with measurement disturbances and ten runs at each iteration are shown in Fig. 4.16(a). The tracking errors exceed the value of $|S| d_{\text {max }}=0.9980 \cdot 10^{-2} \mathrm{rad}$, indicating the presence of disturbances of older iterations in the error signals. The learned feedforward signal of the $25^{\text {th }}$ iteration is shown in Fig. 4.16(b). It can be seen that the learned feedforward signal still contains the disturbance with a frequency of $100 \mathrm{~Hz}$. The amount with which the disturbance is filtered out by taking the mean of 10 error signals depends on the initial phases of the disturbance signals. The amount of disturbance reduction will increase with an increasing number of runs each iteration, but the time needed for each iteration will also increase. A disturbance free feedforward signal is only obtained if the disturbances completely cancel each other. This is not likely to happen in case of random initial phases.

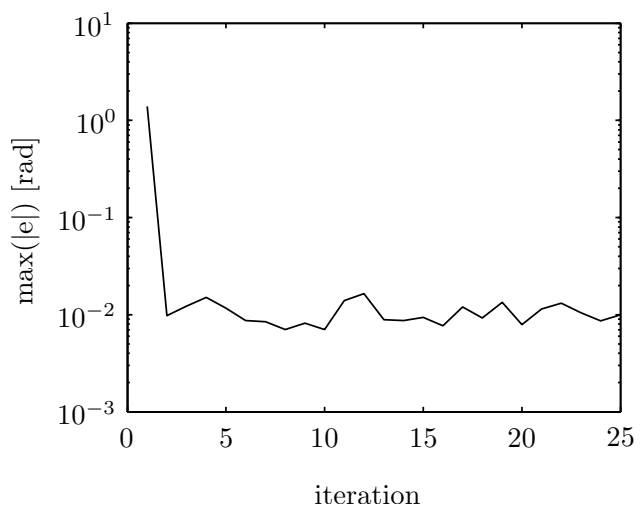

(a) Maximum absolute error

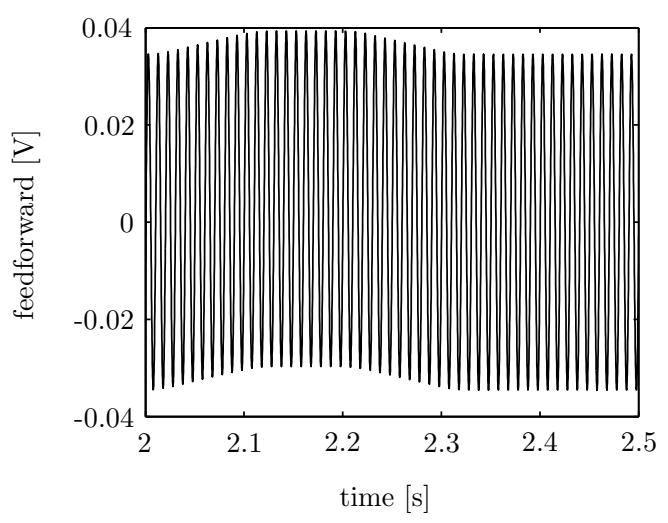

(b) Feedforward signal $k=25$

Figure 4.16: Results averaging with measurement disturbances

Fig. 4.17 shows the results of the simulation with load disturbances. It can be seen that the maximum absolute error exceeds the value of $\left|S_{P}\right| n_{\max }=2.9877 \mathrm{rad}$, indicating that the 
disturbances still affect the learning scheme of ILC. This can also be seen in Fig. 4.17(b), where the feedforward clearly contains the disturbance with a frequency of $1 \mathrm{~Hz}$.

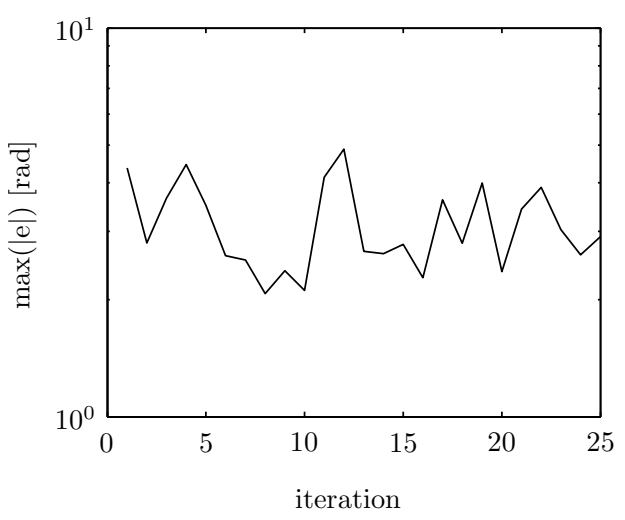

(a) Maximum absolute error

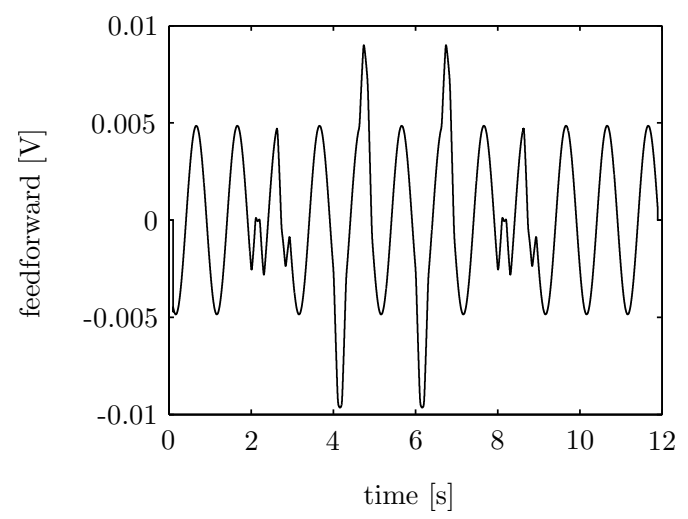

(b) Feedforward signal $k=25$

Figure 4.17: Results averaging with load disturbances

The averaging method removes part of the disturbances since the maximum obtained errors are smaller than the worst case errors found in Section 4.3. However, the disturbances still affect the learning scheme and the learned feedforward, making the averaging method not the most suitable method for removing the non-repetitive part of the error.

\subsection{ILC with wavelet filtering}

In this section the wavelet filtering methods will be tested by means of simulations on the fourth order system of Fig. 4.1. First the settings of the DWT will be discussed. The wavelet filtering methods are tested without disturbances and with both deterministic and stochastic measurement and load disturbances.

\subsubsection{Wavelet filter settings}

The optimal wavelet function is chosen as described in Section 3.2, based on the error between a reference error signal and a reconstructed error signal of the DWT with the two run wavelet filtering method. The optimal wavelet function is determined for the error signals of the first and last iteration of ILC with both deterministic measurement and load disturbances.

The smallest error between the optimal error signal and the reconstructed error signal is obtained with the 'db38' wavelet function [20]. The reference error signal of the first iteration, the reconstructed error signal with deterministic measurement disturbances and the difference between the two signals are shown in Fig. 4.18 for a 'db38' wavelet function. The difference between the two error signals is of order $10^{-6}$ times smaller than the amplitudes of the error signals.

The 'db38' wavelet function has 38 vanishing moments, or 38 number of zero moments of the wavelet function. This is weakly related to the number of oscillations of the wavelet function. The larger the number of vanishing moments, the better the frequency localization of the decomposition. The dependence between wavelet coefficients on different scales decays with increasing wavelet order [6].

The decomposition level determines the lowest frequency that can be observed in the DWT. The decomposition level is chosen such that the approximation coefficients $c A$ contain most of the error of the first iteration without disturbances. The fast Fourier transform (FFT) [20] of the error of the first iteration is shown in Fig. 4.19. The maximum frequency of interest $f_{\text {max, approx }}$ equals $5 \mathrm{~Hz}$. 

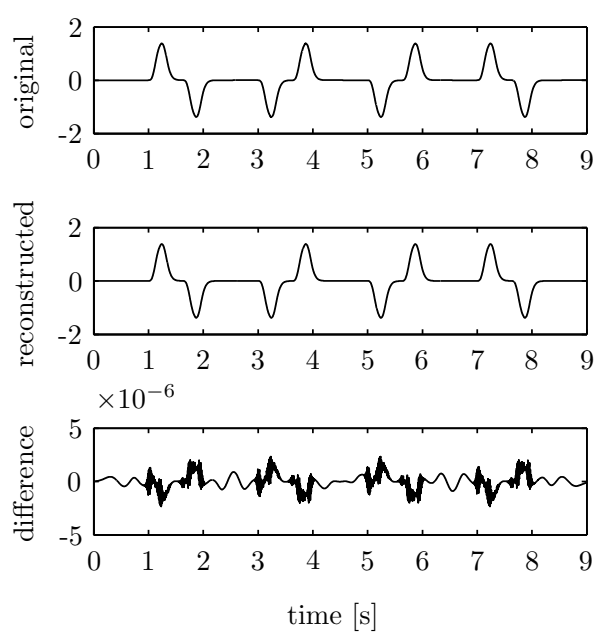

Figure 4.18: error signals in rad of the first iteration for a 'db38' wavelet function

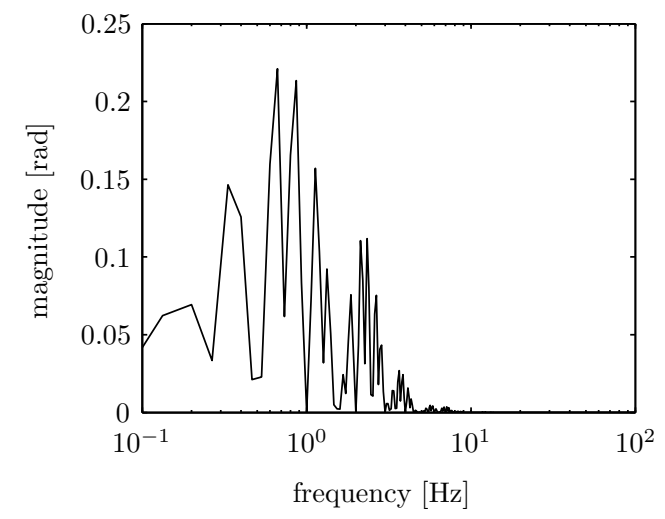

Figure 4.19: FFT error signal $1^{s t}$ iteration without disturbances

For a sampling frequency $f_{s}$ of $8 \mathrm{kHz}$ the decomposition level $p$ can be calculated using (3.5) as

$$
p=\text { floor }\left(\frac{\log \left(f_{s} / f_{\text {max }, \text { approx }}\right)}{\log (2)}-1\right)=9 .
$$

For a decomposition level of 9 and a sampling frequency of $8 \mathrm{kHz}$, the approximation subband $c A$ has a frequency content of $c A \in[0,7.8125] \mathrm{Hz}$.

The threshold level $\gamma$, used in the wavelet filtering methods, consists of a constant part $\gamma_{c}$ and a variable part $\gamma_{v a r}$. The variable part is adjusted based on the wavelet coefficients of the different frequency bands as described in Section 3.2. The constant part is set for the simulations of this section to $\gamma_{c}=5 \cdot 10^{-2}$. With a larger constant part more wavelet coefficients are passed for the reconstruction, however also a larger part of the non-repetitive errors is passed. A smaller constant level removes more coefficients from the reconstruction, but a too small level also removes some of the repetitive part of the error. In this case ILC will not be able to compensate for all repetitive errors, which leads to a larger tracking error after convergence.

\subsubsection{No disturbances}

The wavelet filtering methods, described in Section 3.2, should not affect the performance of the ILC scheme when no disturbances are present. The three different wavelet filtering methods use a different number of runs at the various iterations. The maximum absolute errors are shown in 
Fig. 4.20(b) as a function of both the number of runs, i.e. the number of simulations performed, and as a function of the number of iterations, i.e. each time a feedforward update is performed.

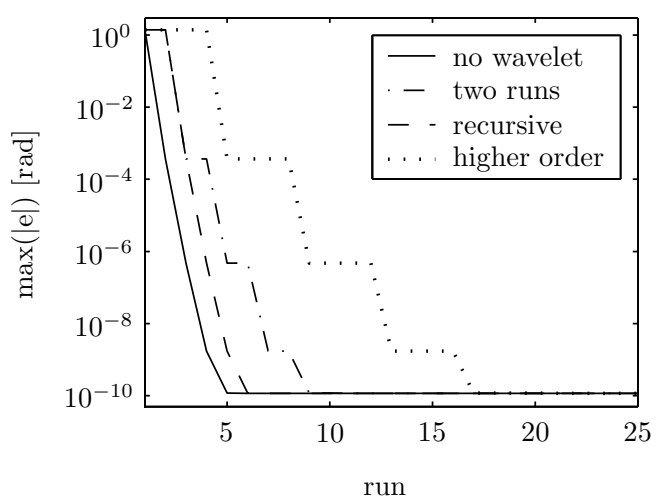

(a) $\max (|\mathrm{e}|)$ as a function of the number of runs

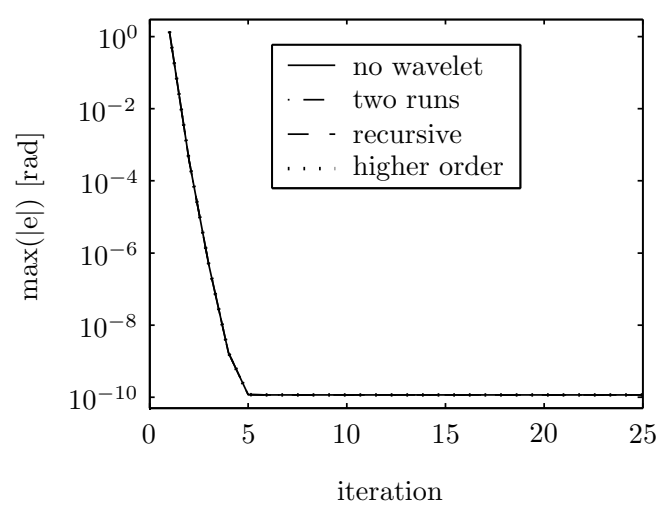

(b) $\max (|\mathrm{e}|)$ as a function of the iteration number

Figure 4.20: results wavelet filtering without disturbances

The maximum absolute tracking errors after convergence are equal for all simulations with and without wavelet filtering. The wavelet filtering methods do not adjust the performance of ILC if no disturbances are present. The wavelet filtering methods all use a different number of runs to converge to the final error as can be seen in Fig. 4.20(a). However, the number of iterations, i.e. the number of feedforward updates, required for convergence is equal, see Fig. 4.20(b). The learned feedforward signals of all simulations are equal, as can be seen in Fig. 4.21. The wavelet filtering methods do not affect the learned feedforward signals in case of no disturbances.

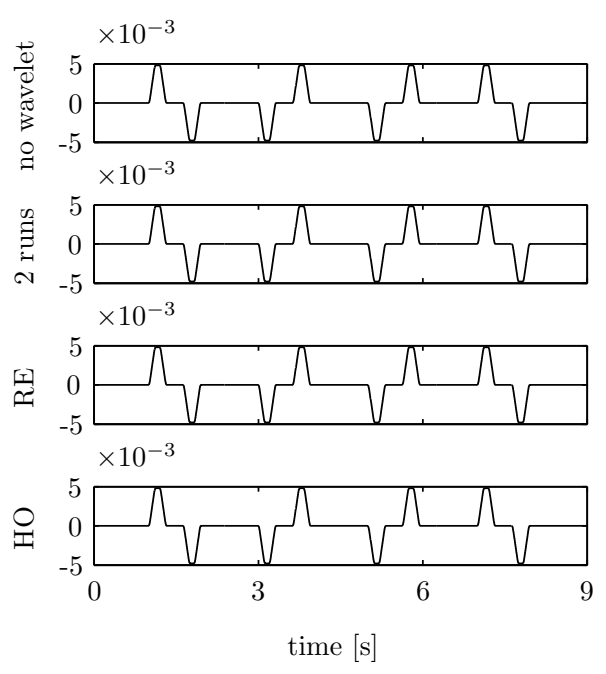

(a) Feedforward signals

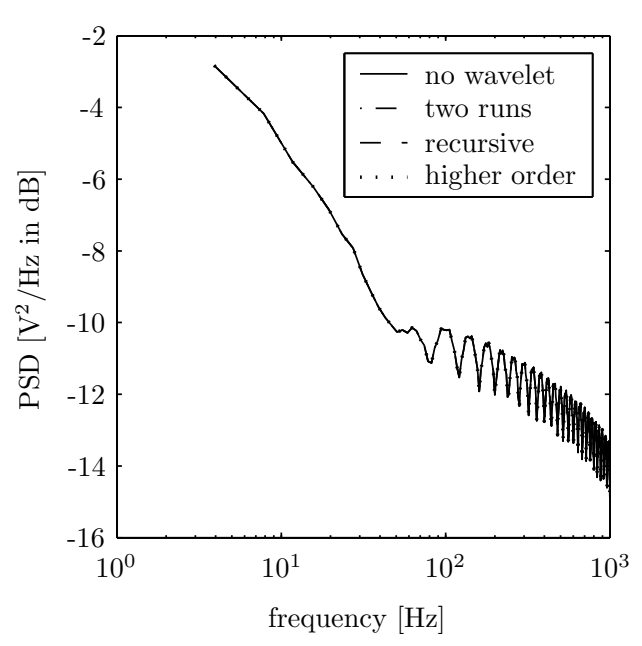

(b) PSDs of the feedforward signals

Figure 4.21: Feedforward signals without disturbances

\subsubsection{Deterministic disturbances}

The performance of the various wavelet filtering methods under harmonic measurement disturbances $d$ and load disturbances $n$ will be investigated in this section. The harmonic disturbances have an initial phase $\phi_{0}$, amplitude $A$ and frequency $f$. The initial phases of the disturbances vary randomly every iteration, the frequency and the amplitude are chosen first constant and 
later random. In order to compare the simulations with the different wavelet filtering methods, random uniformly distributed vectors for the frequency, amplitude and initial phase are generated once a priori and used in all simulations. The random frequencies vary between zero and half the sampling frequency of $8 \mathrm{kHz}$, the random amplitudes between zero and $1 \cdot 10^{-2}$ and the initial phases between 0 and $2 \pi$.

\section{Measurement disturbances}

The maximum absolute errors of the simulations with measurement disturbances, with a constant frequency of $100 \mathrm{~Hz}$ and a constant amplitude of $1 \cdot 10^{-2} \mathrm{rad}$, are shown in Fig. 4.22 for the various wavelet filtering methods. All wavelet filtering methods remove the disturbances from the learning scheme of ILC, leading to a maximum error which converges to $e_{\max }=|S| d_{\max }$. The tracking error without wavelet filtering fluctuates because of the disturbances present in the learning process as can be seen in Fig. 4.22.

The learned feedforward signals for the various simulations are shown in Fig. 4.23(a), all feedforward signals obtained with the various wavelet filtering methods resemble the optimal feedforward signal for the case of no disturbances (see Fig. 4.5(b)). The learned feedforward signal without wavelet filtering is dominated by the disturbances. The power spectral densities (PSDs) of the learned feedforward signals with wavelet filtering show a large reduction around the resonance frequency of $100 \mathrm{~Hz}$ compared to the feedforward signal obtained without wavelet filtering. At low frequencies where the largest part of the error is located (see Fig. 4.19), all PSDs are equal.

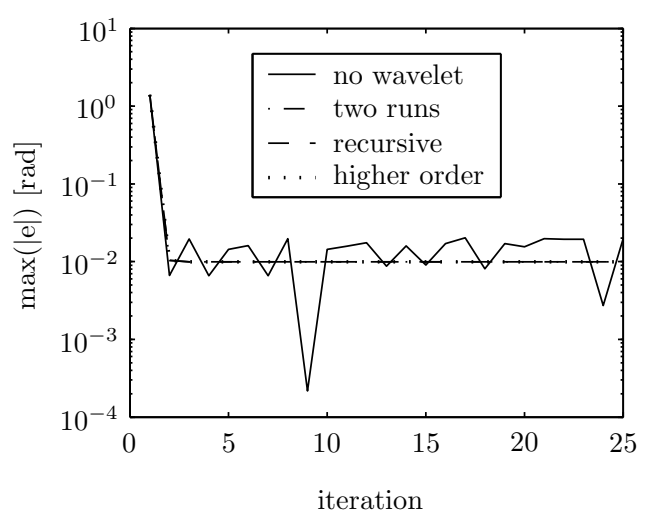

Figure 4.22: Maximum absolute error for measurement disturbances with constant $A$ and $f$

The wavelet filtering methods all realize two major improvements. First, the error shows no amplification above $e_{\max }=|S| d_{\max }$ and secondly, the feedforward signals do not contain any disturbances.

The results of the simulations with random disturbance frequencies and amplitudes are shown in Fig. 4.24. For the wavelet filtering with two runs and the higher order wavelet filtering method, the amplitudes, frequencies and phases of the disturbances of the several runs at equal iterations are also different. The wavelet filtering methods remove the disturbance signals from the tracking errors and the ILC scheme converges to $|S| d_{\max }$. The fluctuation on the errors after convergence is caused by the different disturbance amplitudes and frequencies of the various iterations. The maximum absolute errors of the simulations with wavelet filtering are smaller than the maximum absolute errors without wavelet filtering. The learned feedforward signals with wavelet filtering resemble the optimal feedforward signal as can be seen in Fig. 4.25(a). The PSDs of Fig. 4.25(b) show that the wavelet filtering successfully methods remove the disturbances. At the disturbance frequencies, peaks appear in the PSD of the feedforward signal obtained without wavelet.

The wavelet filtering methods are also able to remove the disturbances from the learning process if one of the variables frequency or amplitude is chosen randomly and the other is constant. 


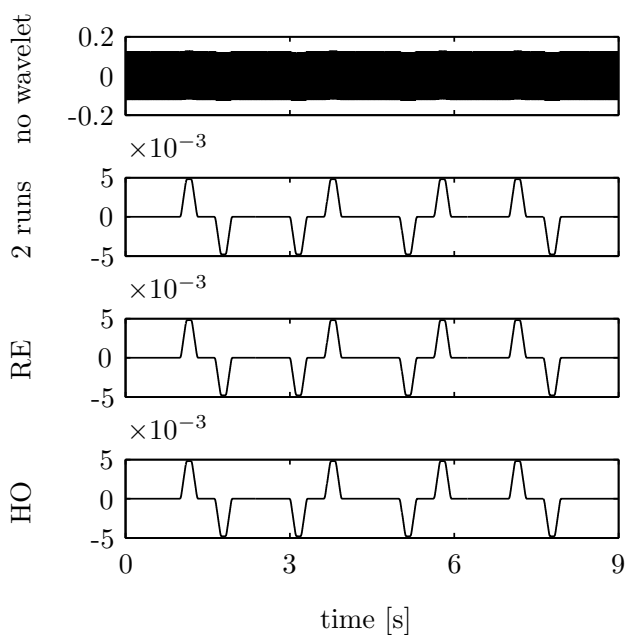

(a) Feedforward signals

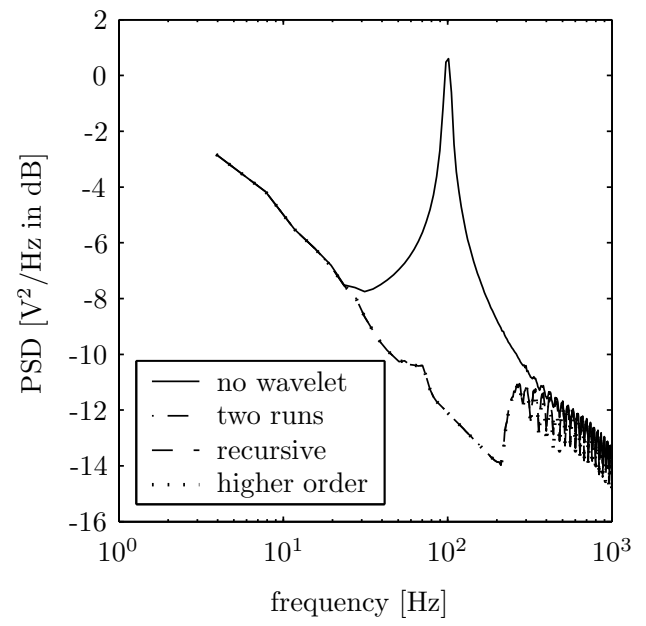

(b) PSDs of the feedforward signals

Figure 4.23: Feedforward signals for measurement disturbances with constant $A$ and $f$

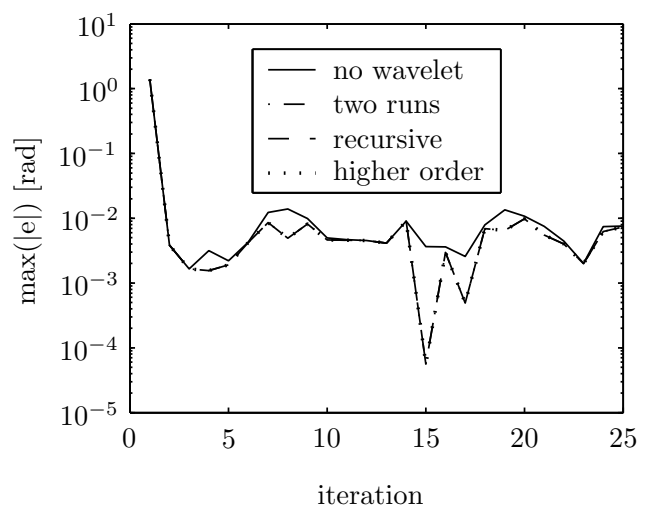

Figure 4.24: Maximum absolute error for measurement disturbances with random $A$ and $f$

\section{Load disturbances}

The simulations with load disturbances with a constant frequency of $1 \mathrm{~Hz}$ and constant amplitude of $1 \cdot 10^{-2} \mathrm{~V}$ result in the maximum absolute tracking errors of Fig. 4.26(a). The disturbance frequency of $1 \mathrm{~Hz}$ is located in the same frequency band as the repetitive part of the tracking error at the first iteration. Since the disturbances dominate the wavelet coefficients, both the disturbances and the repetitive part are removed by the wavelet filtering. This results in a filtered error signal which only contains frequencies outside the lowest frequency band. The learned feedforward signals with wavelet filtering will also contain only frequencies outside the approximation subband.

If the non-repetitive part dominates the error, the resulting feedforward without wavelet filtering is dominated by the disturbances as can be seen in Fig. 4.26(b). In this case, the ILC scheme without wavelet filtering does not converge and an erroneous feedforward signal is learned. When the non-repetitive part is located in the same subband as the repetitive part of the error, learning nothing is $\mathrm{p}$ [referable to learning an erroneous feedforward signal.

An increase in the disturbance frequency to $50 \mathrm{~Hz}$ leads to the maximum absolute errors shown in Fig. 4.27. The wavelet filtering methods all remove the disturbances from the learning 


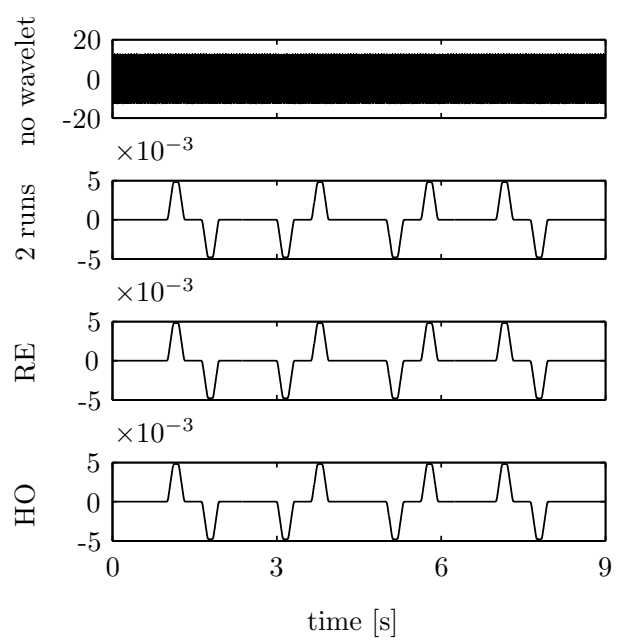

(a) Feedforward signals

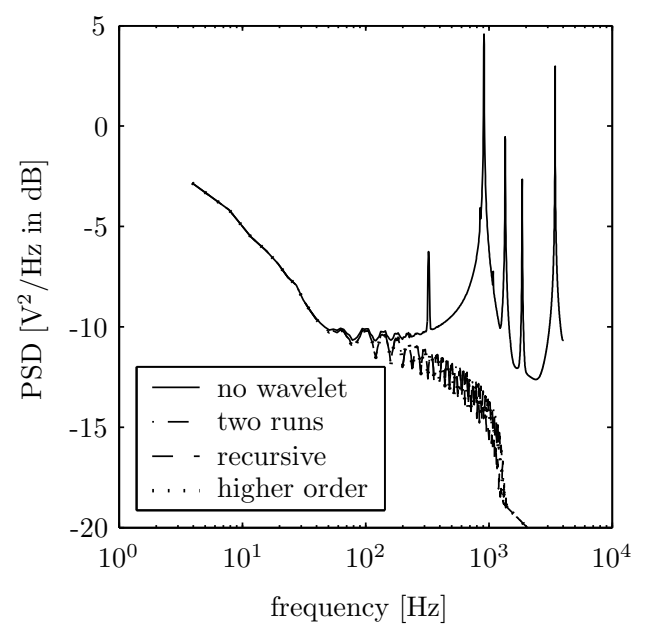

(b) PSDs of the feedforward signals

Figure 4.25: Feedforward signals for measurement disturbances with random $A$ and $f$

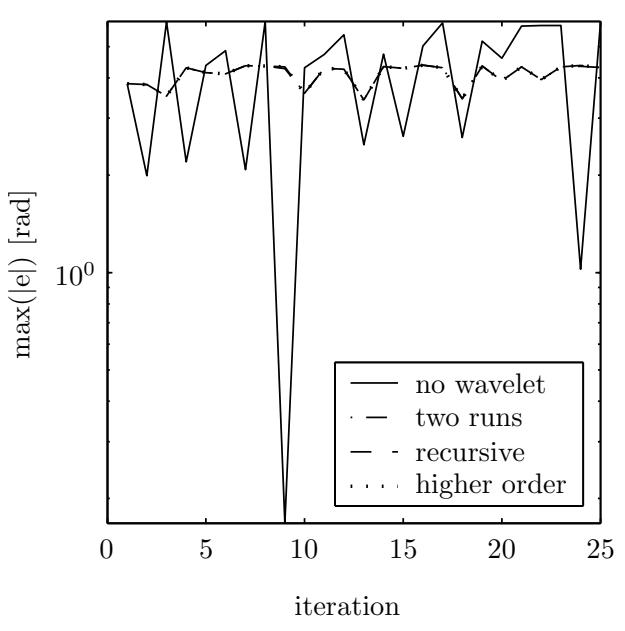

(a) Maximum absolute error

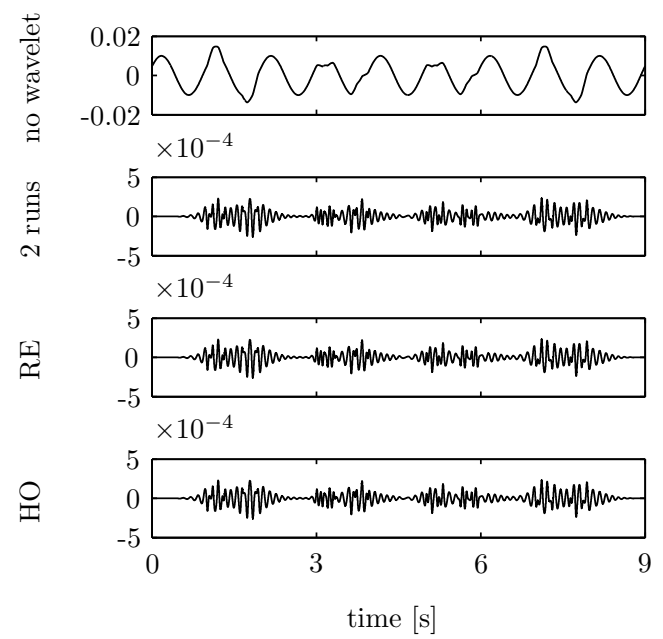

(b) Feedforward signals

Figure 4.26: Results load disturbances with constant disturbance $A$ and $f(1 \mathrm{~Hz})$

scheme. The maximum absolute errors with wavelet filtering converge to $e_{\max }=\left|S_{P}\right| n_{\max }$, whereas the maximum absolute error without wavelet filtering fluctuates because of the presence of the disturbances in the learning scheme. The wavelet filtering methods remove the disturbances with a frequency of $50 \mathrm{~Hz}$ from the feedforward signals, as can be seen in Fig. 4.28.

The simulations with random disturbance frequencies and amplitudes of Fig. 4.29 also show that all wavelet filtering methods remove the disturbances from the learning scheme. The maximum absolute errors converge in case of wavelet filtering to $\left|S_{P}\right| n_{\max }$. Without wavelet filtering, the maximum absolute errors are larger every iteration. The learned feedforward signals with wavelet filtering resemble the optimal feedforward signal. The learned feedforward signal without wavelet filtering contains disturbances as can be seen in Fig. 4.30(a). The PSDs of Fig. 4.30(b) show that the wavelet filtering methods have the largest reduction at high frequencies. 


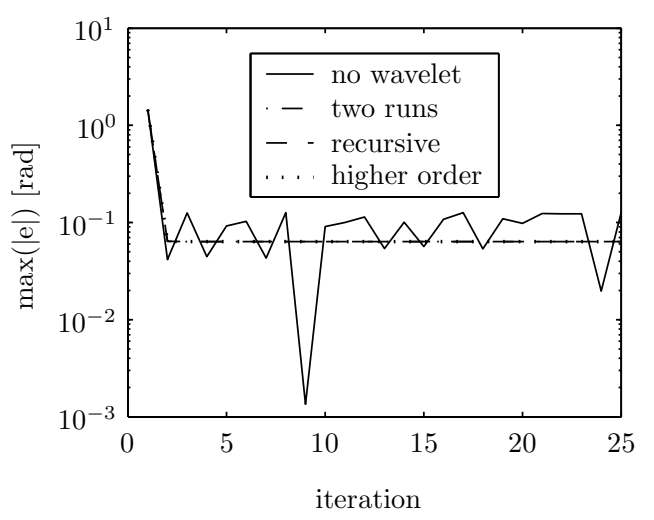

Figure 4.27: Maximum absolute error for load disturbances with constant $A$ and $f(50 \mathrm{~Hz})$

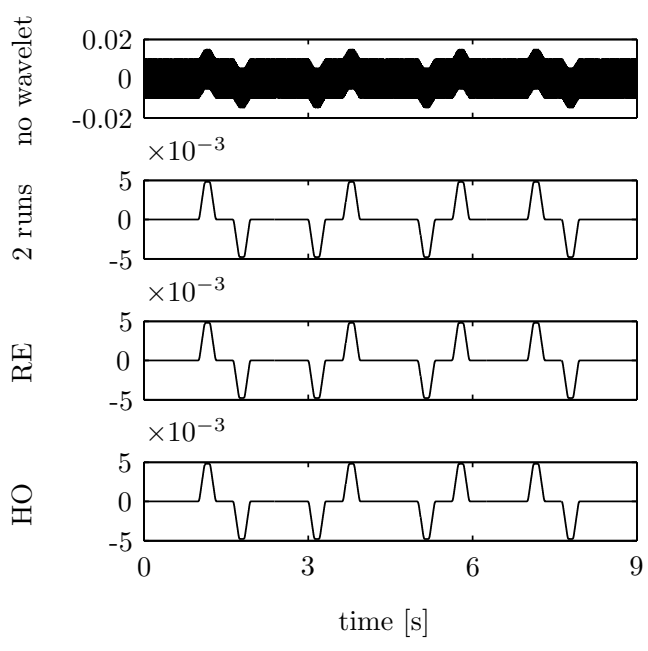

(a) Feedforward signals

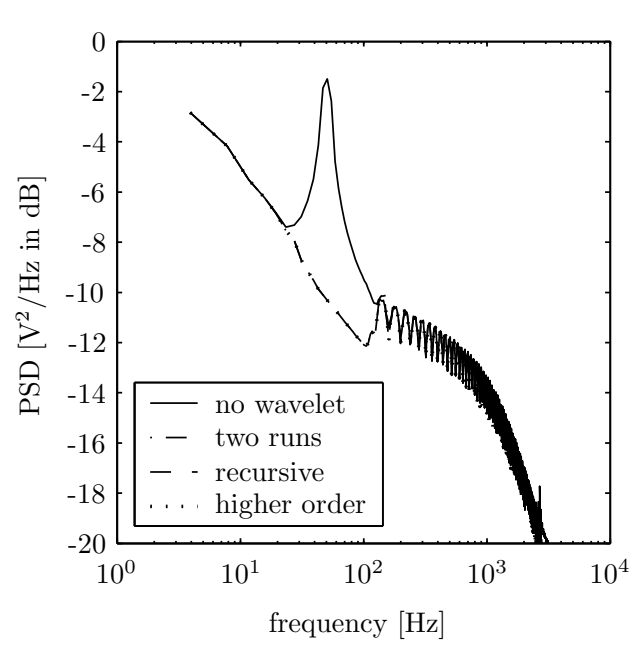

(b) PSDs of the feedforward signals

Figure 4.28: Feedforward signals for load disturbances with constant $A$ and $f(50 \mathrm{~Hz})$

\subsubsection{Stochastic disturbances}

In practice, disturbances are often stochastic, i.e. noisy random processes. The effect of the wavelet filtering methods in the case of noise disturbances is investigated in this section. The noise disturbances are uniformly distributed random signals in the interval [-0.01, 0.01].

For the stochastic disturbances, the root mean square (rms) values are analyzed instead of the maximum absolute errors. The rms values for the simulations with random measurement disturbances are shown in Fig. 4.31. The errors with wavelet filtering are smaller than without wavelet filtering.

The wavelet filtering methods are not able to remove the disturbances entirely from the error. The random disturbances have frequency components at all frequencies, so also in the same frequency band where the repetitive part of the error is located. Because of the scaling of the threshold $\gamma$ due to the variable part $\gamma_{v a r}$ in the wavelet filtering methods, the frequency band where the repetitive part is located is not completely removed from the reconstructed error if the disturbances do not dominate the repetitive part. The reconstructed error signal will contain some part of the disturbances and this also affects the learned feedforward signals of Fig. 4.32(a). The learned feedforward signals of the various wavelet filtering methods show a good correspondence with the optimal feedforward signal, whereas the learned feedforward without wavelet filtering is dominated by the random disturbances. The PSDs of the feedforward signals, shown in Fig. 4.32(b) 


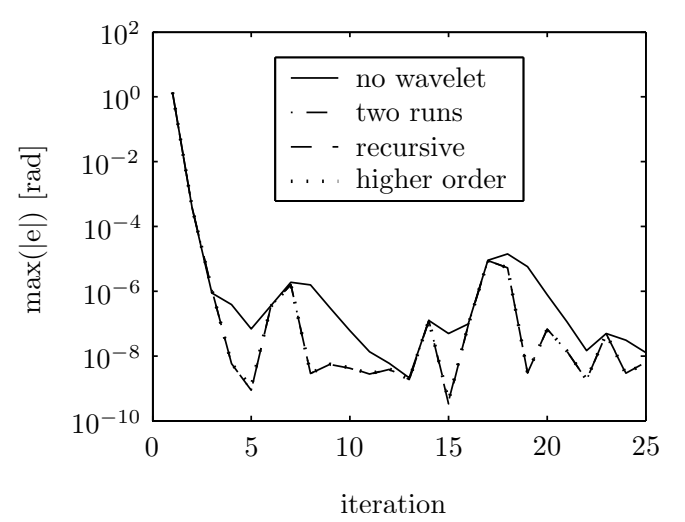

Figure 4.29: Maximum absolute error for load disturbances with random $A$ and $f$

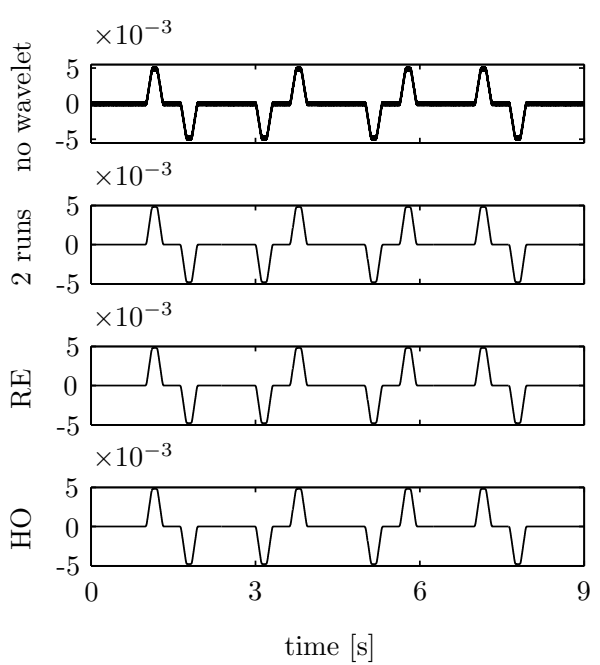

(a) Feedforward signals

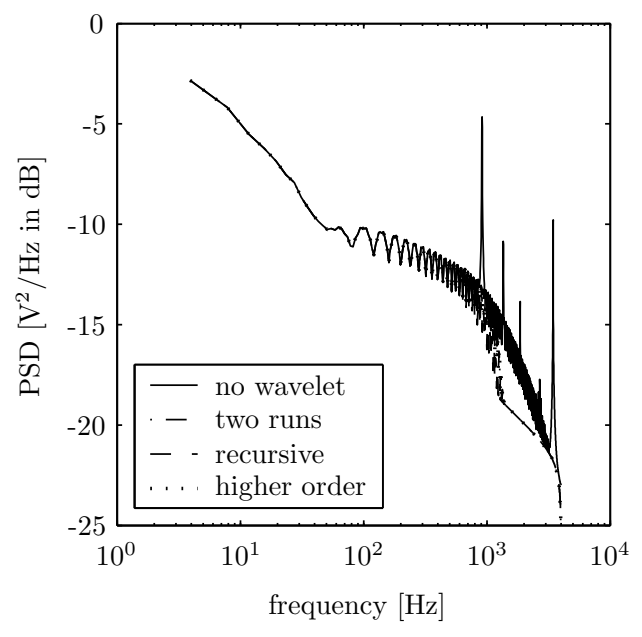

(b) PSDs of the feedforward signals

Figure 4.30: Feedforward signals for load disturbances with random $A$ and $f$

clearly show the reduction of the wavelet filtering methods.

The results for random load disturbances are shown in Fig. 4.33. The rms values of the errors with the various wavelet filtering methods are all smaller than the rms values of the error without wavelet filtering. The load disturbance signals appear in the error filtered with the process sensitivity $S_{P}$ which amplifies the low frequencies in the disturbances signals. Because of this, more disturbance content is present in the lowest frequency band where also the repetitive part of the error is located in the first iteration.

All wavelet filtering methods result in errors which converge to the same order of magnitude. The learned feedforward signals of Fig. 4.34(a) show some differences. Globally, the learned feedforward signals of the simulations with wavelet filtering resemble the optimal feedforward a lot more than without wavelet filtering. Furthermore, all feedforward signals derived with wavelet filtering contain some low-frequent part of the disturbances as discussed before. From the PSDs of the feedforward signals (see Fig. 4.34(b)), it can be seen that the feedforward signal of the recursive method contains more high frequent components than the feedforwards of the other wavelet filtering methods, which is due to the additional filtering by the process sensitivity model. The largest differences between the model and the real system are in the high frequency region. The higher order method is better able to distinguish between the repetitive and non-repetitive part of the error, however the additional runs take a lot more time. 


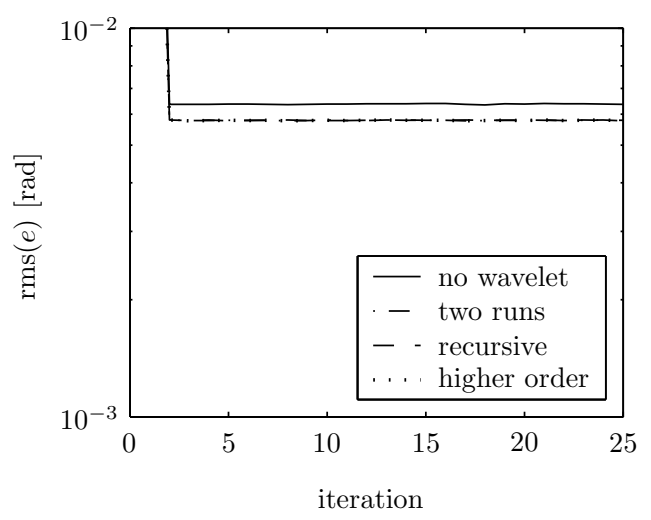

Figure 4.31: Error rms values for random measurement disturbances

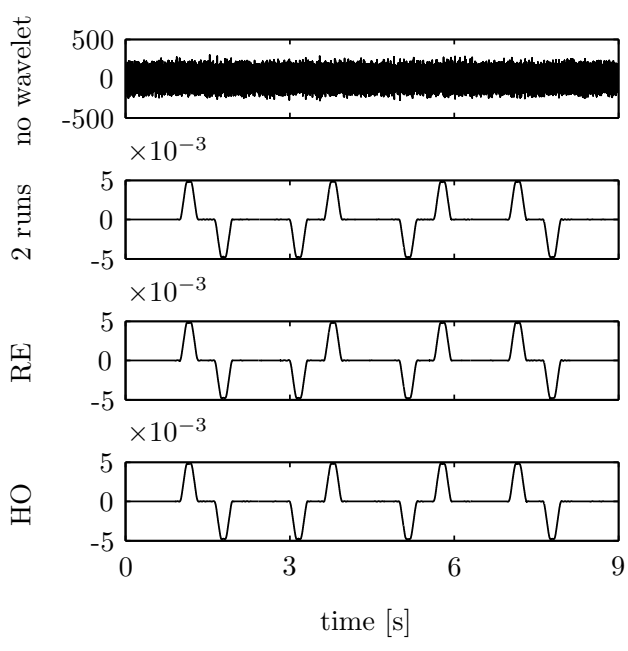

(a) Feedforward signals

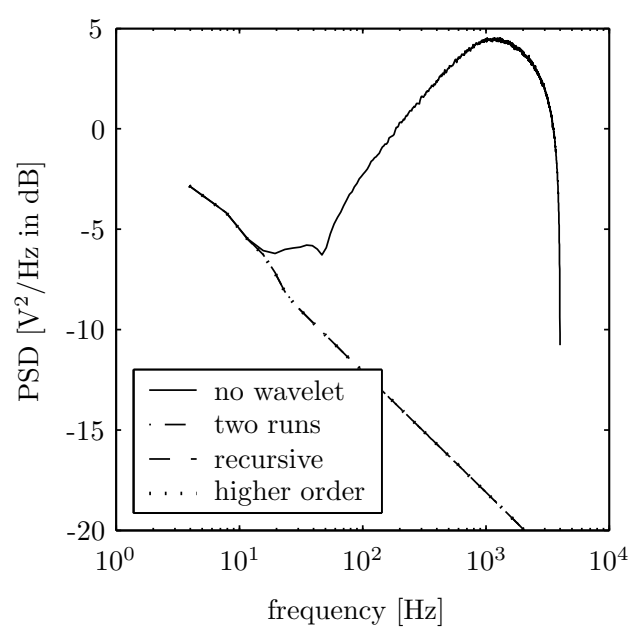

(b) PSDs of the feedforward signals

Figure 4.32: Feedforward signals for random measurement disturbances

The differences between the various methods can best be seen at higher frequencies for load disturbances. The load disturbances appear filtered with the process sensitivity $S_{P}$ in the tracking error, which decays in amplitude at high frequencies. This makes it more difficult to distinguish between the repetitive part and the non-repetitive disturbances at these frequencies. This is not the case for measurement disturbances since these appear in the error filtered with $S$ which has an amplitude of one at high frequencies.

\subsection{Discussion}

The simulations have been performed on a fourth order system, with dynamic properties that are commonly encountered in motion systems. The disturbance analysis simulations have shown that the load and measurement disturbances of the last two iterations determine the tracking error to a great extend. The disturbances appear in the tracking error and with this in the learned feedforward signal. Validation of the derived tracking error expression (2.10) is performed for both deterministic and stochastic disturbances. The calculated maximum errors correspond to the values found in the simulations, showing the validity of the derived expression. A model gain uncertainty and a model order uncertainty influence the tracking error after convergence and the convergence speed of ILC. The effect of model uncertainties can also be analyzed using (2.10). 


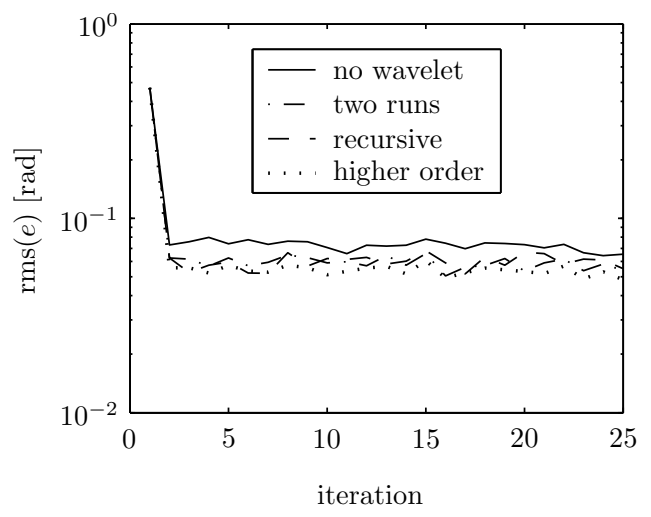

Figure 4.33: Error rms values for random load disturbances

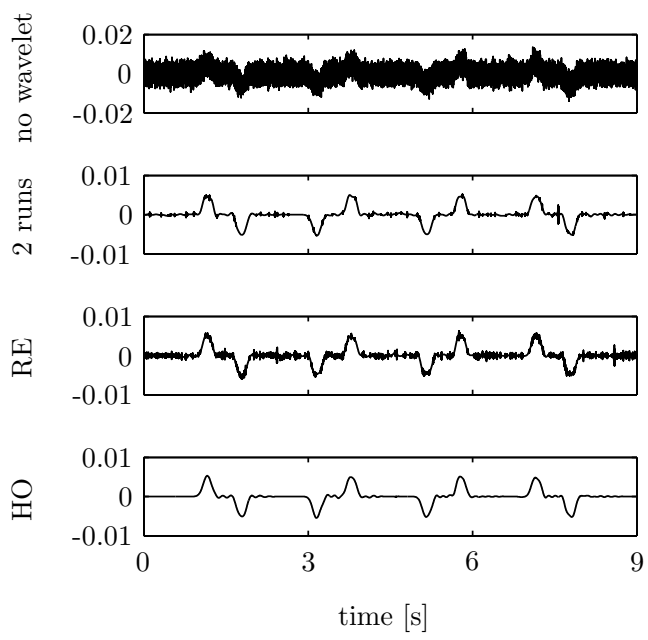

(a) Feedforward signals

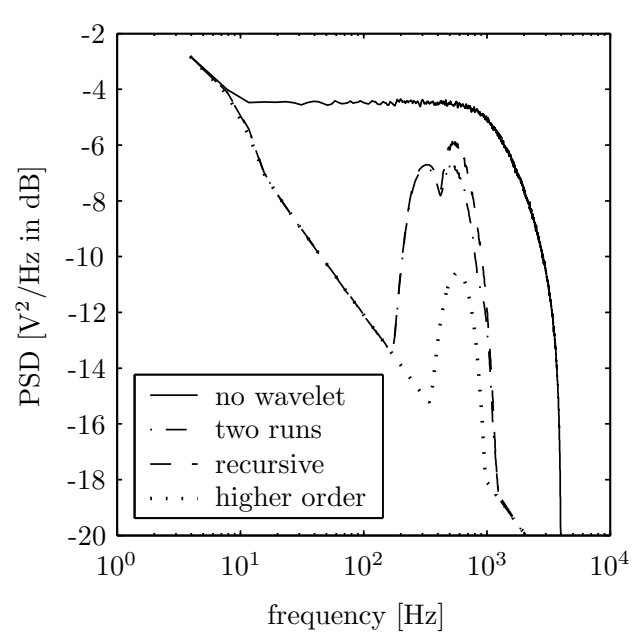

(b) PSDs of the feedforward signals

Figure 4.34: Feedforward signals for random load disturbances

All wavelet filtering methods remove the disturbances from the error signal resulting in a smaller tracking error after convergence than without wavelet filtering, provided that the repetitive and non-repetitive part of the error signal are sufficiently separated in frequency. Without wavelet filtering, the learned feedforward signal is dominated by the disturbances. With wavelet filtering, the feedforward signals contain significantly less disturbances. If the disturbances are located in an equal frequency band as the repetitive part of the error and dominate the wavelet coefficients, both the repetitive part and the disturbances are removed. No feedforward update is performed for the frequencies in that particular frequency band, which is actually the best thing to do in that case.

The performance of the recursive methods depends strongly on the quality of the model of the process sensitivity used to reconstruct the error. If the model is not accurate enough the differences between the simulated error and reconstructed error are large and no (or a small) feedforward update is performed.

The higher order method shows an improvement to the two run and recursive method in the sense that less disturbances are present in the learned feedforward signal. The small improvements in the feedforward signal do not lead to a smaller tracking error. The amount of additional runs needed for the higher order method do not counterweight against the improvements. However the higher order method can be exploited further by designing more similarity criteria with the available signals of the four runs. 


\section{Chapter 5}

\section{Experiments}

In this chapter an experimental validation of the designed wavelet filtering methods will be performed. In mechanical (motion) systems, the non-repetitive disturbances are mainly caused by actuator disturbances and measurement disturbances, i.e. quantization noise. These disturbances are located at high frequencies. Therefore the benefit of wavelet filtering can be found for these systems especially at higher frequencies. For classic ILC, when learning up to high frequencies, the error and feedforward signal become dominated by noise while the convergence criterion is still met. Wavelet filtering makes it possible to learn up to high frequencies and derive a feedforward signal that contains almost no disturbances. This should result in an smaller tracking error and improved performance of ILC.

ILC with the different wavelet filtering methods will be implemented on three different systems. In Section 5.1, experiments will be performed on an academic system, namely a flexible shaft system. The wavelet filtering methods will be applied to a relatively simple and a complex industrial system in respectively Section 5.2 and Section 5.3. The sections discuss the problems encountered and the results of the various experiments

\subsection{Flexible shaft system}

The flexible shaft system, shown in Fig. 5.1, consists of two rotating masses which are connected through a flexible shaft. The first mass is excited by a motor and the position of the second mass is measured using an incremental encoder. The position can be measured with a resolution of $3.1416 \cdot 10^{-3} \mathrm{rad}(2000$ increments per revolution).

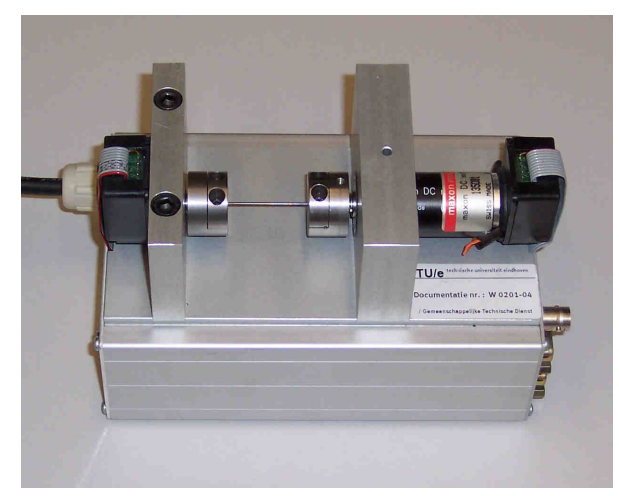

Figure 5.1: Flexible shaft system 
Fig. 5.2 shows the measured and fitted FRFs of the flexible shaft system. The system FRF shows at $10 \mathrm{~Hz}$ a small ripple in the magnitude and a noise burst in the phase, which is caused by cogging. This ripple shifts with the rotational speed used during the FRF measurement. The input of the system is limited at $\pm 2.5 \mathrm{~V}$.
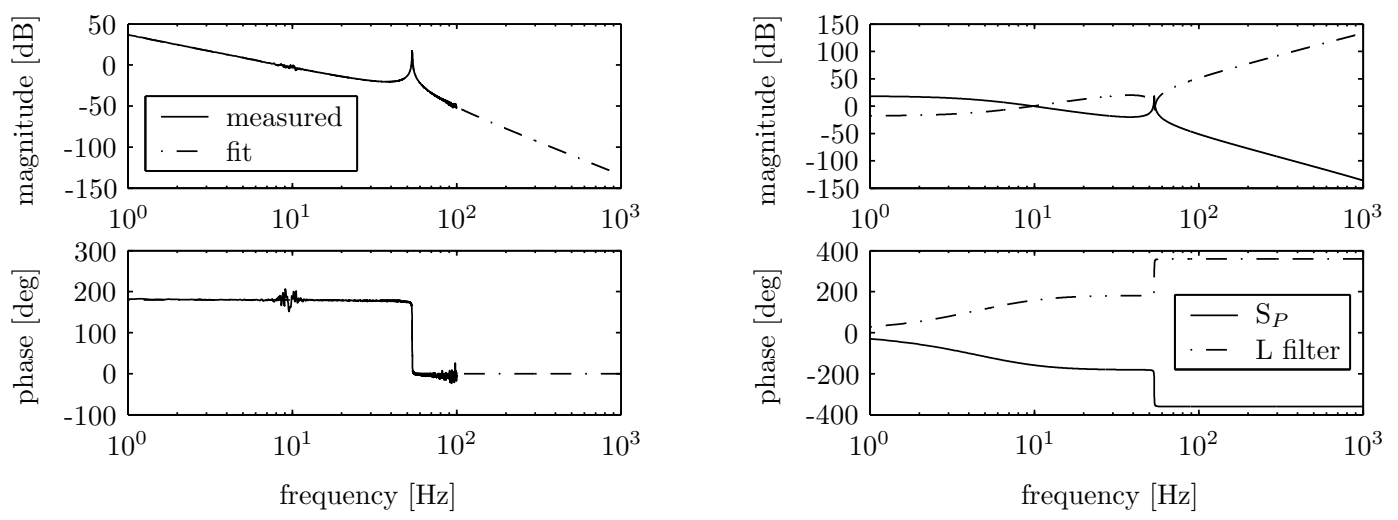

Figure 5.2: Measured and fitted FRFs

Figure 5.3: FRFs discrete $S_{P}$ and $L$ filter

Above $100 \mathrm{~Hz}$, the system FRF cannot be measured correctly, which is due to the limited input voltage $u(t)$ and limited resolution of the position measurement $y(t)$ of the system. The output $y$ of the system is determined by the system $P$ and input $u$ as $y=P u$. With knowledge of the maximum used input during the FRF measurement $u_{\max }$ and the measurement resolution $y_{\text {res }}$, the minimum magnitude of the system FRF that can be measured can be calculated. For the FRF measurement of Fig. 5.2, the maximum input voltage equals $2.48 \mathrm{~V}$, the minimum measurable magnitude now equals

$$
|H|_{\min }=\frac{y_{\text {res }}}{u_{\max }}=\frac{3.1416 \cdot 10^{-3}}{2.48}=1.2646 \cdot 10^{-3}=-57.96 \mathrm{~dB} .
$$

This value is reached at approximately $100 \mathrm{~Hz}$ as can be seen in Fig. 5.2. The maximum input voltage is also reached at this frequency.

Using the fit of the system, a feedback controller is designed which results in a bandwidth of the controlled system of $5 \mathrm{~Hz}$. The controller samples at $8 \mathrm{kHz}$. The learning filter of Fig. 5.3 is obtained using the ZPETC method [36] and a discrete model of the process sensitivity $S_{P}$. The $Q$ filter is chosen as a sixth order low-pass filter with a cut-off frequency of $125 \mathrm{~Hz}$. The convergence criterion $\left|Q\left(1-L S_{P}\right)\right|<1$ is shown in Fig. 5.4. It can be seen that the convergence criterion is met for all frequencies.

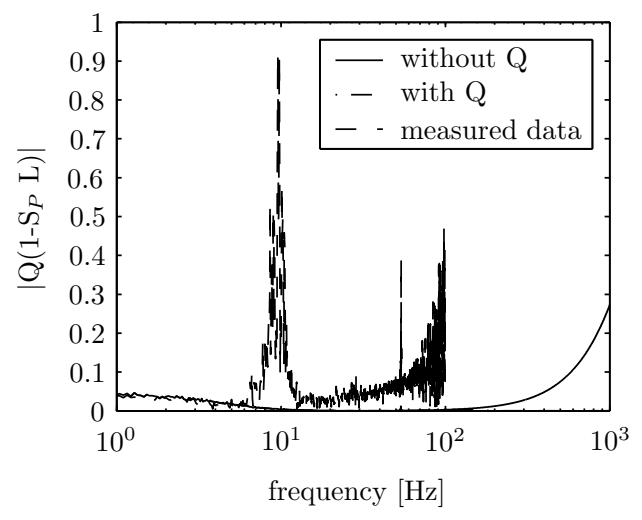

Figure 5.4: Convergence criterion 


\subsubsection{Disturbance analysis}

In the disturbance analysis of Section 2.2, an expression for the tracking error of an arbitrary iteration of ILC as a function of the reference, the measurement and the load disturbances is derived (2.10). With this expression, the influence of disturbances and model errors on the tracking error can be evaluated. This expression has been validated by means of simulations in Section 4.3. The experimental validation of (2.10) will be performed using the flexible shaft system of Fig. 5.2 by adding additional known measurement and load disturbances, both deterministic and stochastic. Experimental validation is also performed for model gain and order uncertainties. The results can be found in Appendix $\mathrm{C}$ for measurement and load disturbances and in Appendix D [22] for model uncertainties. The experiments shows that (2.10) correctly describes the tracking error in the presence of disturbances and model uncertainties.

\subsubsection{Applicability of ILC}

ILC derives a feedforward for a fixed reference signal. The system should be at the same initial position every run in order for the errors to be repetitive. Therefore a homing procedure is used which puts the system in a well-defined initial position. The flexible shaft system resembles the system used for the simulations of Chapter 4. The reference signal used for the experiments is equal to the reference of the simulations as shown in Fig. 4.4. The reference signal covers a range of a total of $20 \pi \mathrm{rad}$, i.e. ten whole rotations. The minimum error which can be reached is limited by the measurement resolution and equals $3.1416 \cdot 10^{-3} \mathrm{rad}$.

In order to see wether ILC can be useful for this system, ten tracking experiments are performed. During the ten experiments no feedforward controller is used and the tracking error is measured. An average error of the ten tracking errors is calculated as is shown in Fig. 5.5(a). The non-repetitive part of the first experiment is calculated by subtracting the average part from the measured tracking error, of which the non-repetitive part is shown in the bottom figure of Fig. 5.5(a). It can be seen that the non-repetitive part is approximately a factor 100 smaller than the repetitive part of the error.

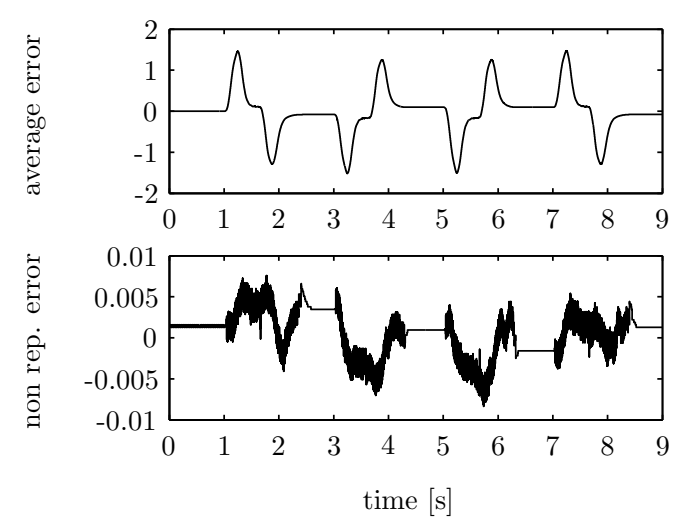

(a) time signals

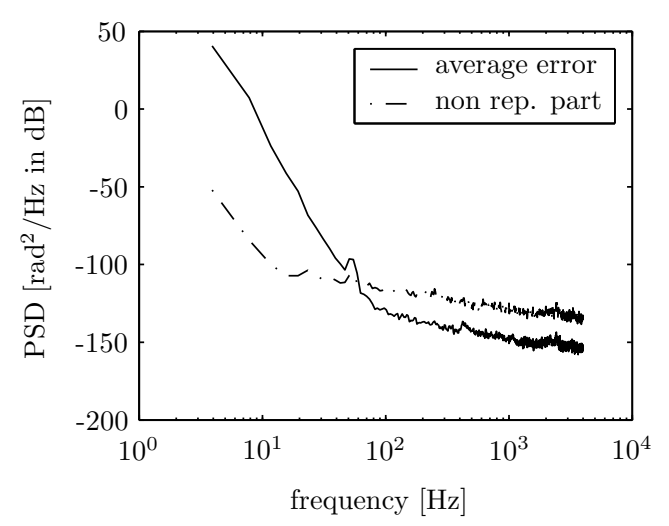

(b) power spectral density

Figure 5.5: Average and non-repetitive part first tracking error

In Fig. 5.5(b) the power spectral density (PSD) of the average error and the non-repetitive part of the error of the first experiment are shown. It can be seen that the PSD of the average error is larger than the PSD of the non-repetitive error up to a frequency of approximately $60 \mathrm{~Hz}$. For frequencies larger than $60 \mathrm{~Hz}$, the non-repetitive part dominates the error, resulting in a limited performance of classic ILC. 
The ten tracking experiments show that there exists a large repetitive part of the error. ILC can therefore be applied on the flexible shaft system to reduce this repetitive part and improve the tracking performance. There exists also a non-repetitive part of the error which limits the performance of ILC.

\subsubsection{Results of ILC}

The settings of the DWT for the wavelet filtering methods are determined as described in Chapter 3. The constant threshold level $\gamma_{c}$ equals $5 \cdot 10^{-2}$. The decomposition level is set to 9 by using (3.5) with $f_{s}=8 \mathrm{kHz}$ and $f_{\text {max,approx }}=5 \mathrm{~Hz}$. These settings correspond to the settings used in the simulations of Chapter 4 .

The rms values of the errors obtained without wavelet filtering and with the various wavelet filtering methods are shown in Fig. 5.6(a). The rms value of the error for the experiment without wavelet filtering converges to a value of approximately $6 \cdot 10^{-3} \mathrm{rad}$, which corresponds to approximately two counts. The classic ILC scheme reduces the error by approximately a factor 100 . However, note that this system is an academic system which is especially designed to have nice dynamic properties and that a relatively low bandwidth of the feedback loop is used.

The errors with all wavelet filtering methods converge to approximately one count, which is smaller than without wavelet filtering. The wavelet filtering methods remove the non-repetitive part of the error which is present in the system, leading to a smaller error after convergence and an improved ILC performance.

The recursive method has horizontal parts in the convergence, this indicates that in these iterations no feedforward update is performed. This is caused by the difference between the measured error signals and the reconstructed error signals at these iterations. The linear model of the $S_{P}$ does not describe the system dynamics accurately enough to reconstruct the error signal. For example, the friction present in the system is not incorporated in the linear model. Because of the large difference between the measured and the reconstructed error signals, the complete error is removed by the wavelet filtering and no feedforward update is performed. In the next iteration, the feedforward update equals zero and two different measured signals are compared by the coefficient adjustment method (see also (3.15)). This causes the recursive method to degrade to the two run method in the sense that an equal amount of runs is needed to reach convergence.

The higher order method shows in Fig. 5.6(a) no improvement over the two run method. The learned feedforward signals of the two methods converge to an error which is approximately equal.

The reduction of the tracking error by the various filtering methods can be demonstrated more clearly by comparing the PSDs of the error signals of the last iteration as shown in Fig. 5.6(b). It can be seen that the PSD of the experiment without wavelet filtering shows a large peak around the resonance frequency. This is caused by the presence of a resonance in the process sensitivity $S_{P}$. Non-repetitive disturbances at frequencies around this resonance frequency are amplified. The wavelet filtering methods have the greatest reduction around the resonance frequency and at high frequencies where the non-repetitive disturbances become dominant.

The learned feedforward signals of the various experiments are shown in Fig. 5.7. Without wavelet filtering, the feedforward is dominated by the non-repetitive disturbances. The feedforward signals of the experiments with wavelet filtering are not dominated by noise. The feedforward signal of the recursive method resembles the feedforward of the two run method, this is also expected since the recursive method degraded in performance to the two run method. The PSDs of the feedforward signals are shown in Fig. 5.7(b). The feedforward of the higher order method shows some improvement with respect to the two run method and the recursive method. However the improvement does not lead to a smaller tracking error, as shown in Fig. 5.6. The feedforward signals obtained with wavelet filtering contain significantly less high frequency content. At low frequencies, where the largest part of the error is located, the feedforward signals are equal. The feedforward signals obtained with wavelet filtering still contain the repetitive part of the error at high frequencies. 


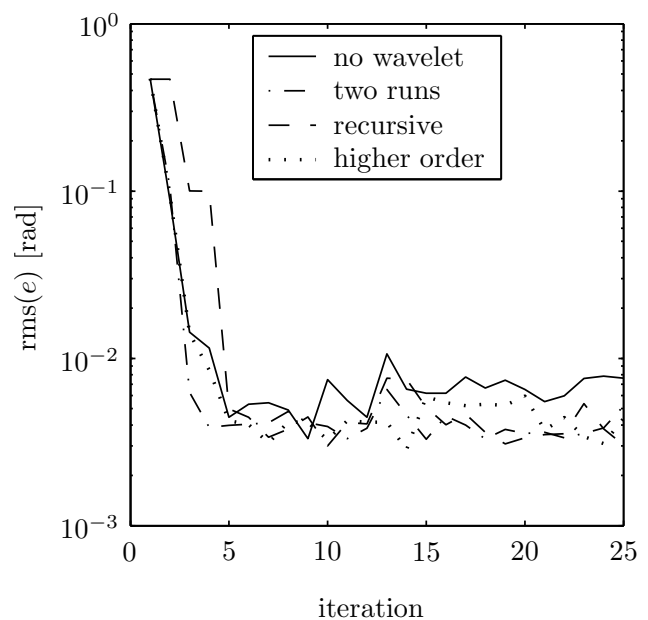

(a) Error rms values

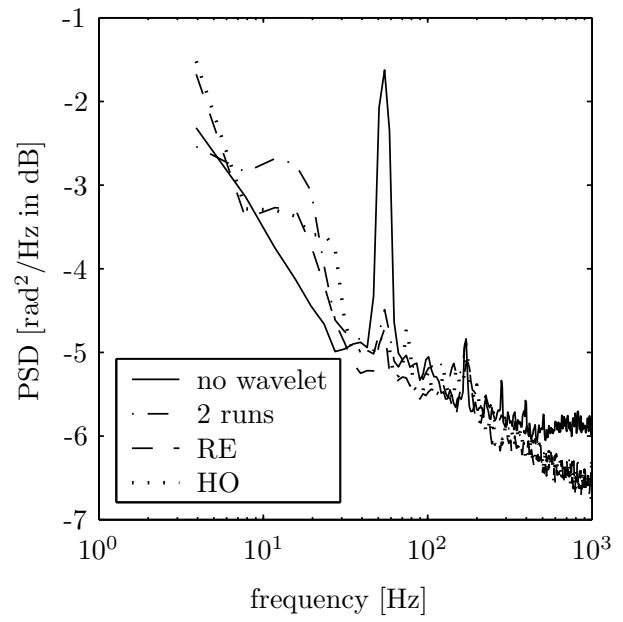

(b) PSDs of the error signals of $k=25$

Figure 5.6: Error signals of the flexible shaft system

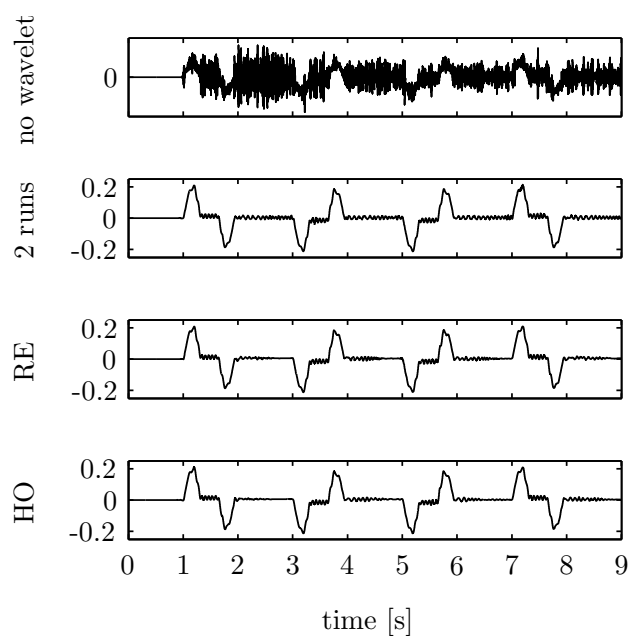

(a) Feedforward signals

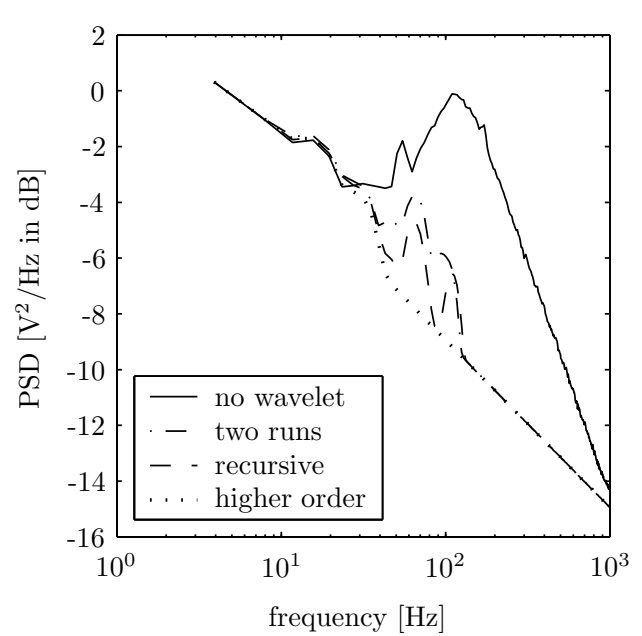

(b) PSDs of the feedforward signals

Figure 5.7: Feedforward signals of the flexible shaft system

\subsection{Printer}

The second system on which experiments are performed is a HP inkjet printer, shown in Fig. 5.8. The printhead is driven using a motor and a driving belt. The position of the printhead is measured using a linear incremental encoder. The measured and fitted FRFs of the motor to the position of the printhead are shown in Fig. 5.9. The measured FRF is reliable up to a frequency of approximately $150 \mathrm{~Hz}$, above this frequency the measured FRF is dominated by quantization noise. The minimum measurable amplitude of the FRF can be calculated as discussed in Section 5.1. The minimum measurable position is one count, the maximum input voltage of the FRF measurement equals $2.49 \mathrm{~V}$, the minimum measurable value of the FRF can be calculated as

$$
|H|_{\min }=\frac{y_{\text {res }}}{u_{\max }}=\frac{1}{2.49}=4.0161 \cdot 10^{-1}=-7.924 \mathrm{~dB}
$$

This level corresponds to the level obtained in the FRF measurement of Fig. 5.9 at $150 \mathrm{~Hz}$.

A homing procedure is used to position the printhead at the start of every experiment at a 


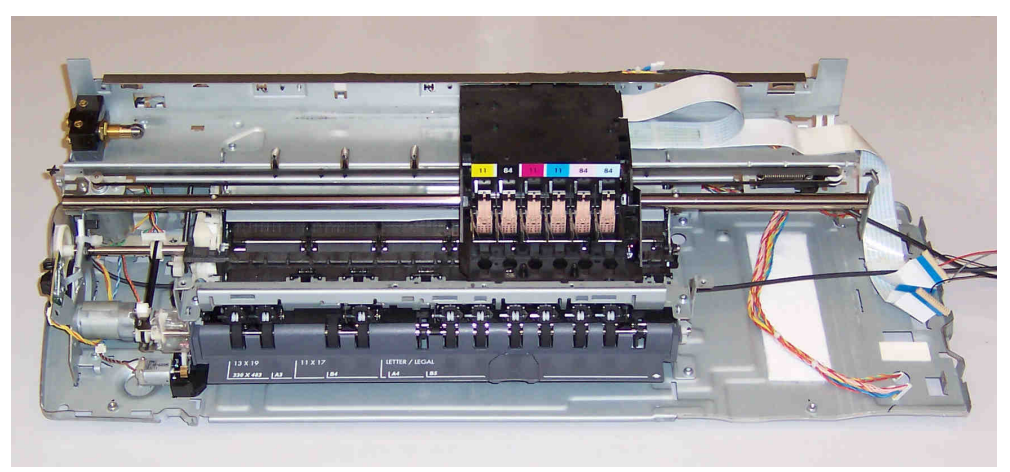

Figure 5.8: Printer

well-defined initial position. The usefulness of ILC on the printer system is tested by performing ten tracking experiments with the reference shown in Fig. 5.11. Of the ten experiments, the average and the non-repetitive part of the error are calculated in the same way as done for the flexible shaft system of Section 5.1. The PSDs of the average and non-repetitive error show that the repetitive part dominates the error up to a frequency of approximately $90 \mathrm{~Hz}$.
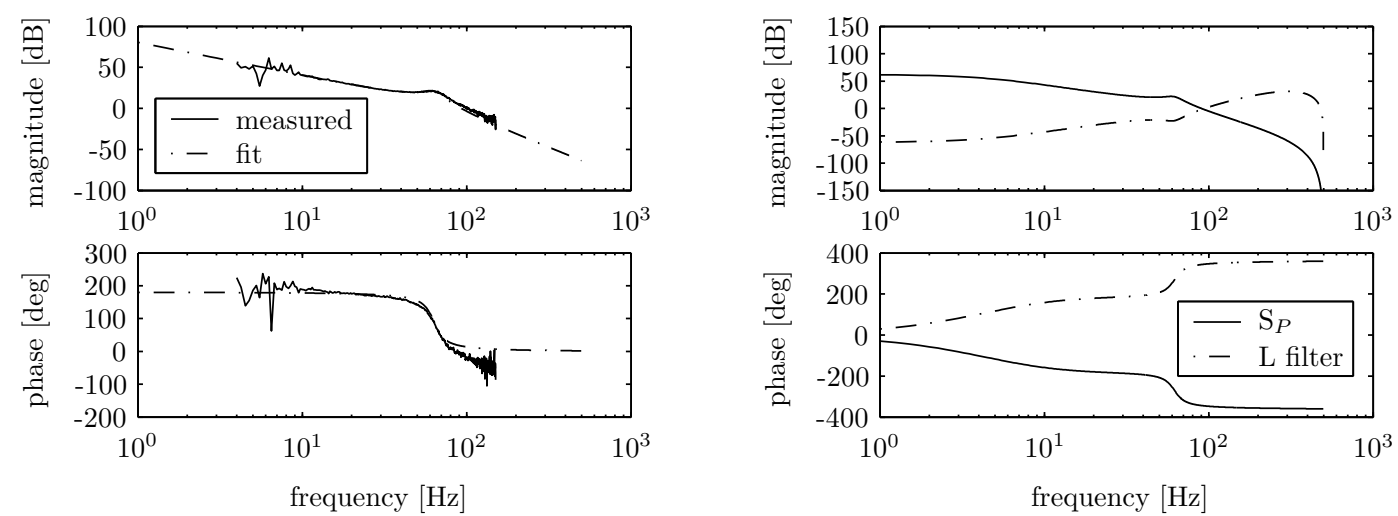

Figure 5.9: Measured and fitted FRFs

Figure 5.10: FRFs discrete $S_{P}$ and $L$ filter

The learning filter $L$ of Fig. 5.10 is designed using a discrete model of $S_{P}$ and the ZPETC method [36]. In order to satisfy the convergence criterion of (2.4), a fourth order low-pass $Q$ filter with a cut-off frequency of $120 \mathrm{~Hz}$ is used (see Fig. 5.12). Note that this frequency is located above the frequency where the non-repetitive part of the error becomes dominant. The increase in the convergence criterion calculated with the measured data around $100 \mathrm{~Hz}$ is caused by the noise on the FRF measurement of Fig. 5.9 at these frequencies.

The experiments with the various wavelet filtering methods are performed with a constant threshold level $\gamma_{c}=5 \cdot 10^{-2}$. The decomposition level is calculated using (3.5) with $f_{s}=1 \mathrm{kHz}$ and $f_{\text {max }, \text { approx }}=3 \mathrm{~Hz}$, the decomposition level of the DWT equals seven.

Fig. 5.13(a) shows the rms values of the error for the various experiments. The error without wavelet filtering converges to a value of approximately eight counts. The rms values of the error with the various wavelet filtering methods are all smaller. The higher order wavelet filtering method has a comparable performance to the two run method: both converge to a value of approximately three counts. The recursive method shows horizontal parts in the convergence, which was also the case for the flexible shaft system. The linear model of $S_{P}$ is not able to fully describe the real system behavior. This results in large differences between the measured and reconstructed error signals and no feedforward update is performed. The performance decays to the two run method. However the recursive method converges to a somewhat larger value, 


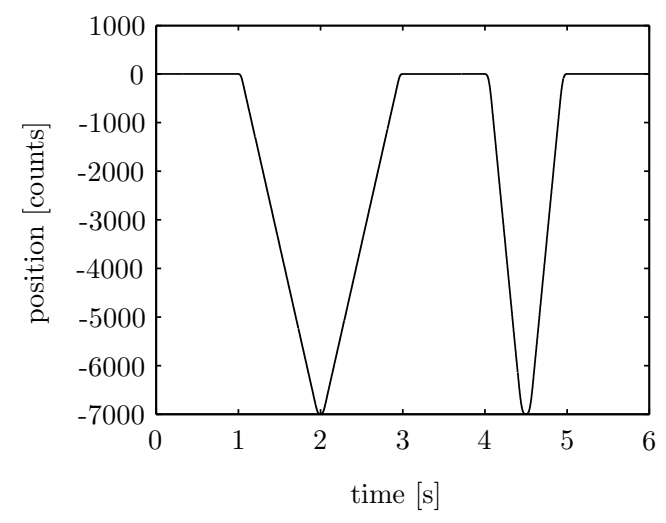

Figure 5.11: Reference signal printer

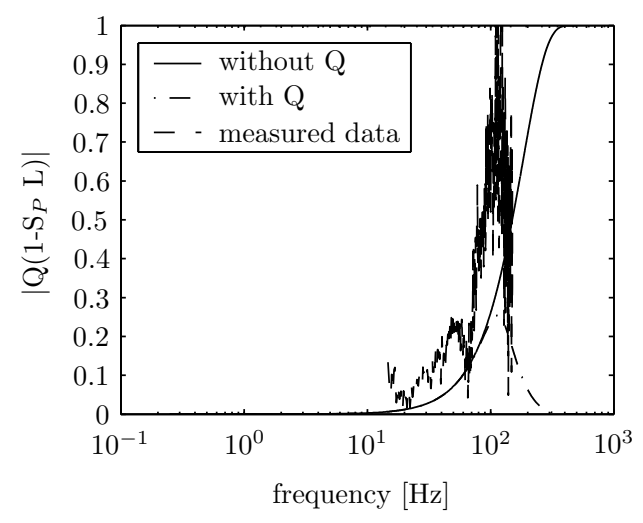

Figure 5.12: Convergence criterion

indicating that some of the repetitive part of the error is also filtered out. This occurs because, after convergence, some small feedforward update is made each iteration. With this the two error signals to be compared will always show some differences. The performance of the recursive method is highly sensitive to the quality of the model of the process sensitivity $S_{P}$. The obtained error after convergence is still smaller than without wavelet filtering.

The PSDs of the error signals of the last run are shown for all experiments in Fig. 5.13(b). The wavelet filtering methods lead to tracking errors with a reduced frequency content in almost the whole frequency range. The largest benefit is achieved in the region where the non-repetitive part becomes dominant. Above $150 \mathrm{~Hz}$, the frequency content of the error signals of all experiments is too small to be measured correctly and no improvement can be made. Furthermore it can be seen that the recursive method leads to a somewhat larger PSD than the two run method and the higher order method. This corresponds to the results of Fig. 5.13(a).

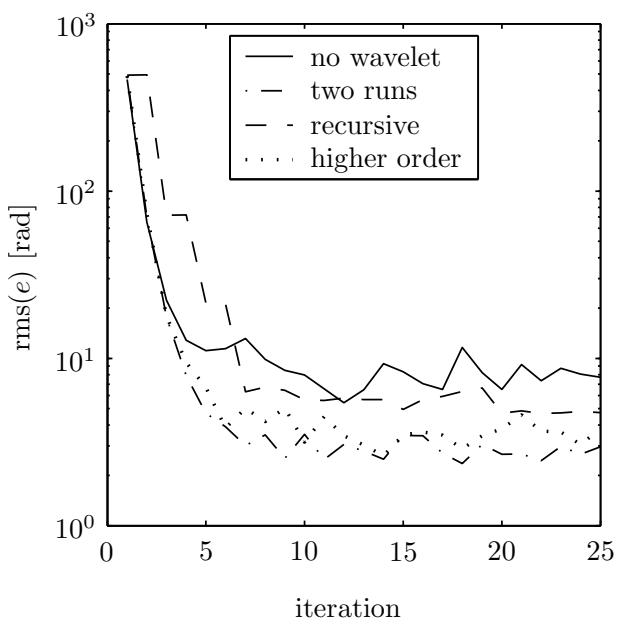

(a) Error rms values

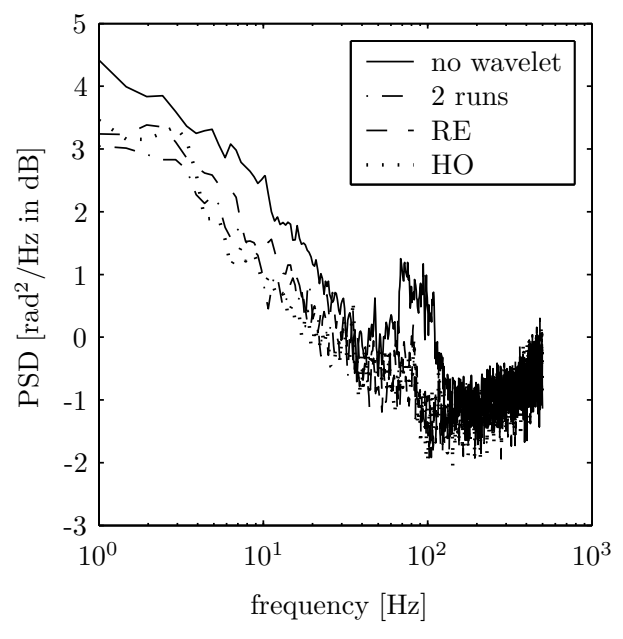

(b) PSDs of the error signals of $k=25$

Figure 5.13: Error signals of the printer

The learned feedforward signals of the various experiments can be found in Fig. 5.14(a). The feedforward signal learned without wavelet filtering is dominated by noise. Where the feedforward signals from the various wavelet filtering methods are not. The feedforward signals of the two run method and the recursive method are nearly equal. The PSDs of the feedforward signals of Fig. 5.14(b) show that the PSD of the feedforward signal of the recursive method is larger than the PSD of the feedforward signal of the two run method. This corresponds to the results of 

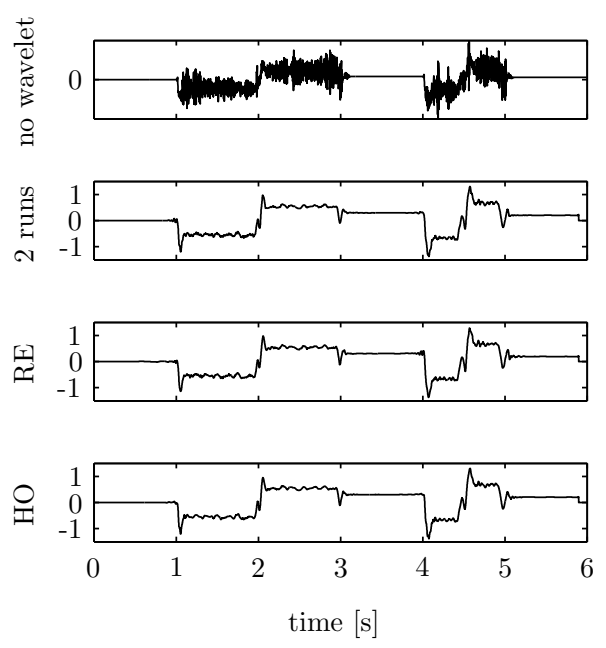

(a) Feedforward signals

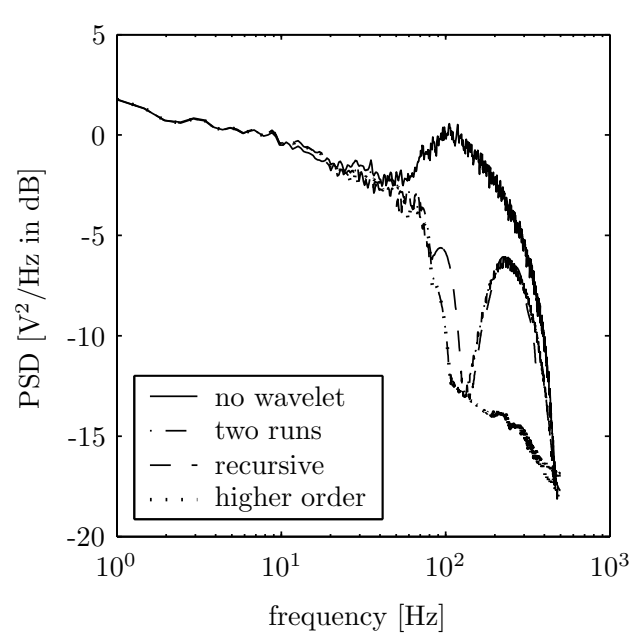

(b) PSDs of the feedforward signals

Figure 5.14: Feedforward signals of the printer

Fig. 5.13(a). The feedforward signal of the higher order method shows some improvement to the other feedforward signals in the sense that less disturbances are present, however the improvements are not that high in comparison to the amount of additional runs required. Furthermore the improvements are not large enough to lead to a smaller tracking error.

\subsection{Pizzabot}

Finally, the wavelet filtering methods are tested on a complex industrial system, a so called pizzabot which is shown in Fig. 5.15. The pizzabot is a transposer robot with an arm which can move in four degrees of freedom. The arm is driven by four different motors of which the position is measured by incremental encoders. The concept of ILC and the wavelet filtering methods are applied to the rotational motion of the system.

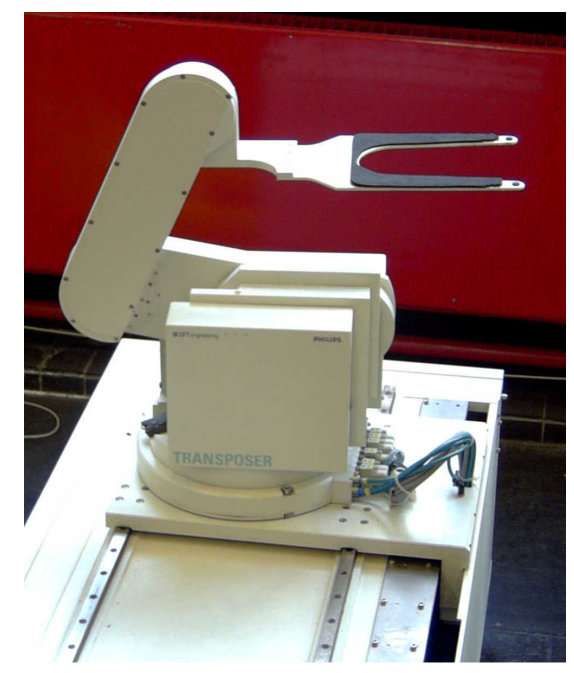

Figure 5.15: Pizzabot

The measured system FRF is shown in Fig. 5.16. The FRF shows a couple of resonances and anti-resonances in the frequency range from $40 \mathrm{~Hz}$ up to $100 \mathrm{~Hz}$. Furthermore, an anti-resonance 

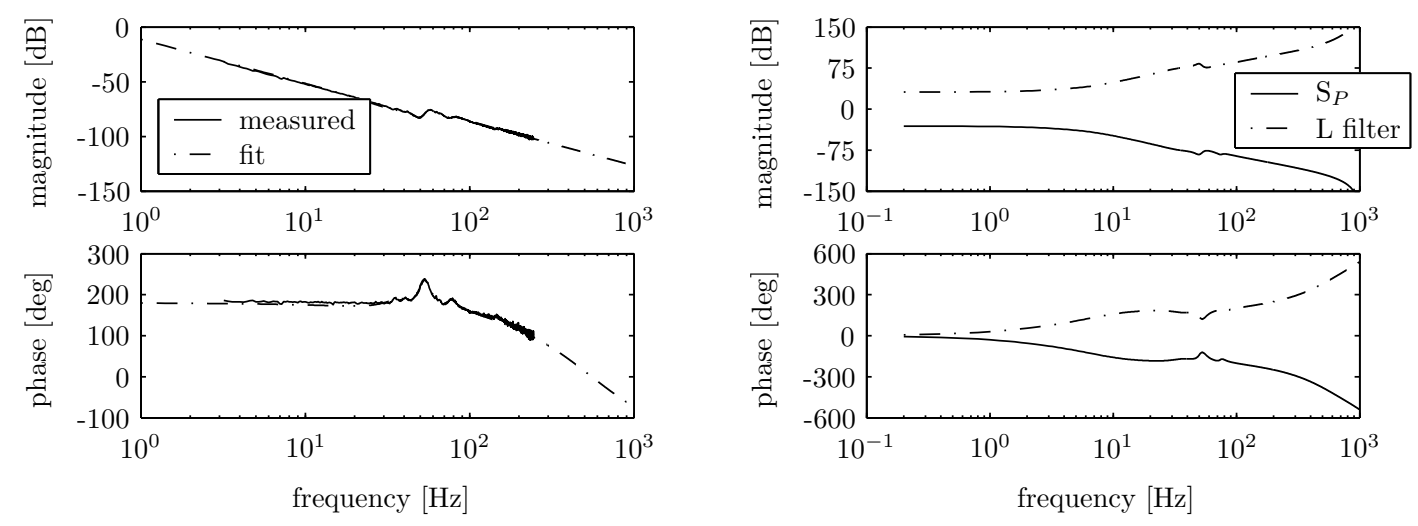

Figure 5.16: Measured and fitted FRFs

Figure 5.17: FRFs discrete $S_{P}$ and $L$ filter

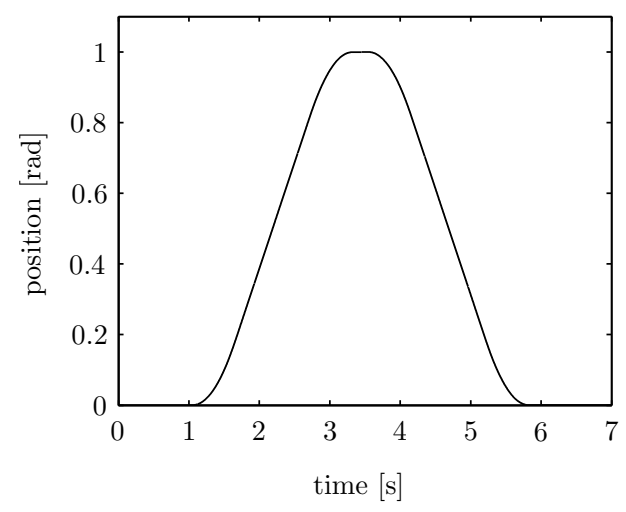

Figure 5.18: Reference signal pizzabot

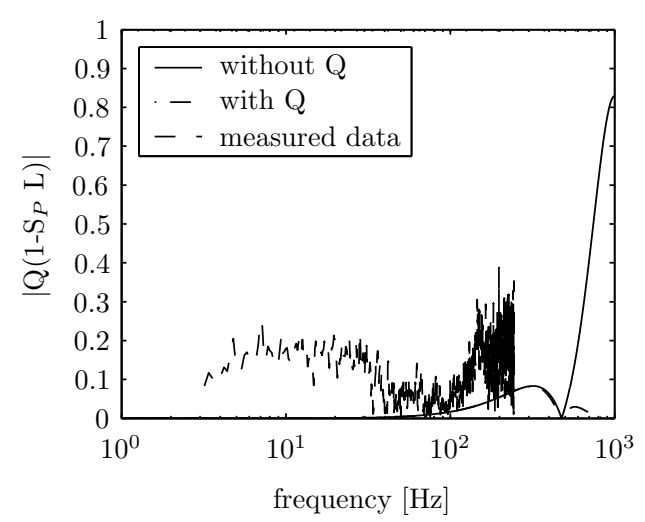

Figure 5.19: Convergence criterion

and resonance are present around $150 \mathrm{~Hz}$. Using the fit of the system FRF (see Fig. 5.16), a feedback controller $C$ is designed which results in a bandwidth of the controlled system of $5 \mathrm{~Hz}$, the controller samples at $2 \mathrm{kHz}$. The designed learning filter $L$ is shown in Fig. 5.17. In order to fulfill the convergence criterion (2.4), a fourth order low-pass $Q$ filter with $f_{c}=450 \mathrm{~Hz}$ is used (see Fig. 5.19). The reference signal to be tracked is shown in Fig. 5.18.

The order and cut-off frequency of the $Q$ filter are determined by the quality of the $L$ filter and thus by the quality of the fit of the system FRF. In order to learn up to high frequencies, a good quality fit is required. The sampling frequency used to measure the system FRF also plays a role in the performance of ILC on the pizzabot. The design of the learning filter $L$ with the ZPETC method [36] results in an amplitude distortion at high frequencies (see also Appendix A). Since the resonances are located at high frequencies, a low sampling frequency results in a distortion of the learning filter $L$ around the resonance frequencies. This limits the performance of ILC and the remaining tracking error is determined by the incorrectly learned repetitive part of the error. In this case, the tracking error converges to a larger value and the wavelet filtering methods do not improve the performance, nor make it worst. Therefore a sampling frequency has to be used which does not lead to a significant amplitude distortion at low frequencies and around the resonances. In order to start each experiment at a fixed initial position, a homing procedure is used.

The decomposition level of the DWT for the wavelet filtering methods is set to eight, this value is obtained using (3.5), a sampling frequency of $f_{s}=2 \mathrm{kHz}$ and $f_{\text {max } \text {,approx }}=3 \mathrm{~Hz}$. The constant level of the threshold $\gamma_{c}=10 \cdot 10^{-3}$. The threshold is a bit larger than for the experiments of the other two systems. This is done because of the presence of some fluctuations in the homing procedure. The differences in the initial position after homing lead to differences in the repetitive part of the error. 
The experiments without wavelet filtering and with the various wavelet filtering methods lead to rms values of the error signals as shown in Fig. 5.20(a). All wavelet filtering methods lead to errors which are smaller than without wavelet filtering. The recursive method again shows horizontal parts due in the convergence to the same reasons as for the flexible shaft system and the printer, i.e. a not accurate enough model of $S_{P}$.

The PSDs of the error signals of the last iteration are shown in Fig. 5.20(b). The PSDs of the error signals with the wavelet filtering methods show a significant reduction of the energy over the whole frequency range compared to the PSD of the experiment without wavelet filtering.

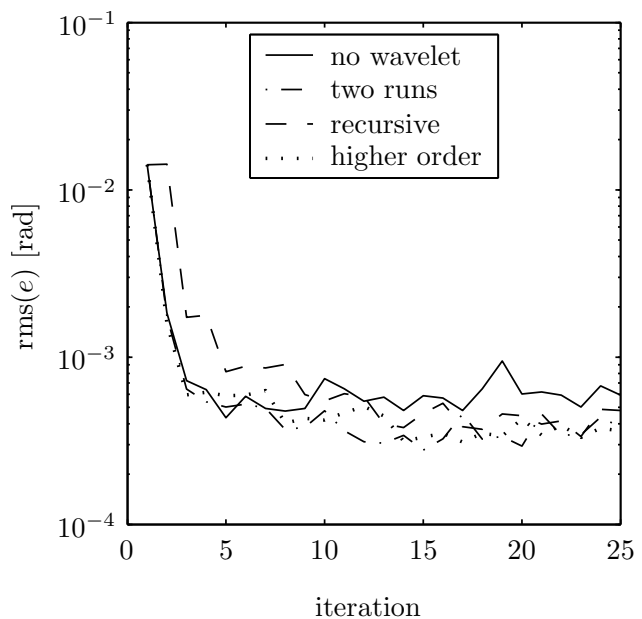

(a) Error rms values

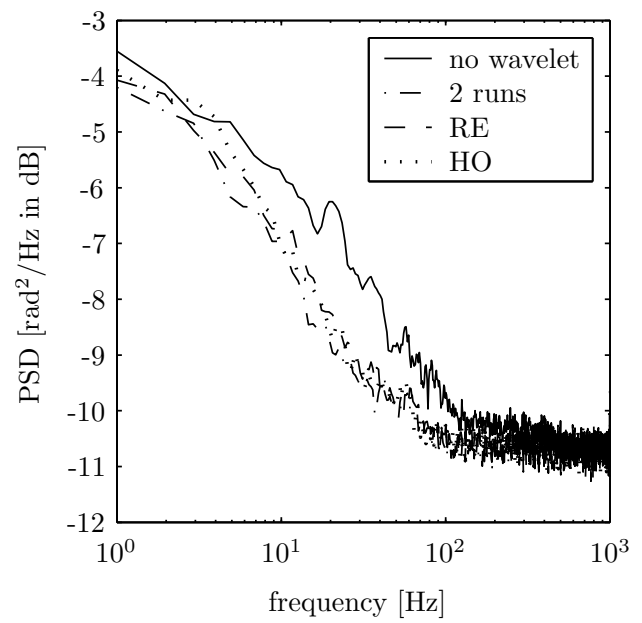

(b) PSDs of the error signals of $k=25$

Figure 5.20: Error signals of the pizzabot
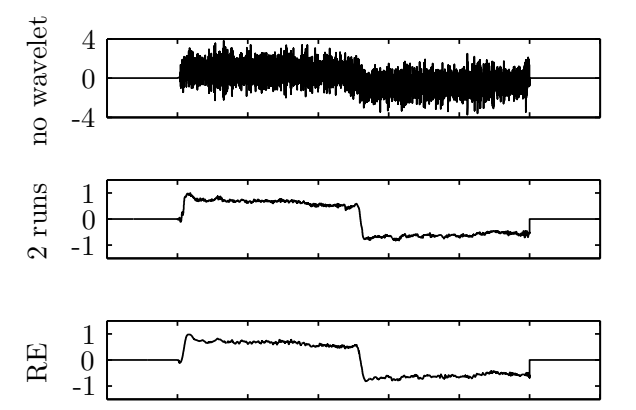

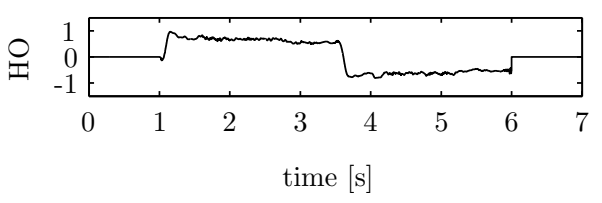

(a) Feedforward signals

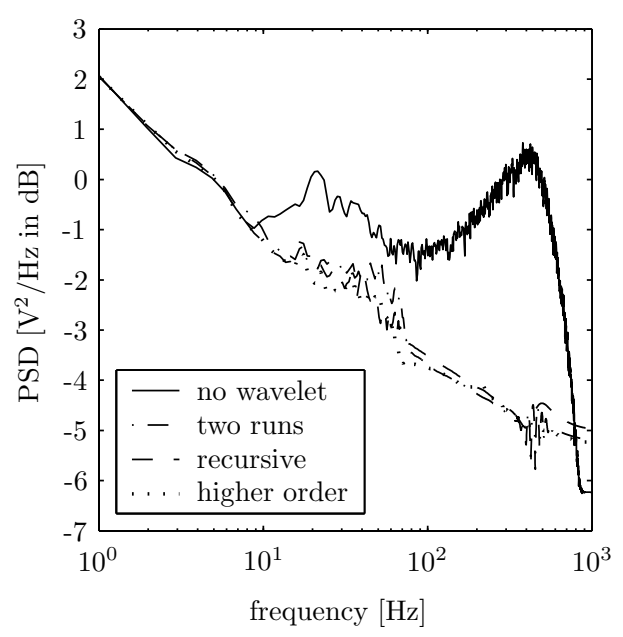

(b) PSDs of the feedforward signals

Figure 5.21: Feedforward signals of the pizzabot

The feedforward signals of Fig. 5.21(a) and the PSDs of Fig. 5.21(b) show that without wavelet filtering the feedforward is dominated by noise, which is not the case with the various wavelet filtering methods. The higher order method contains marginally less noise than the other two wavelet filtering methods, however this does not lead to a smaller tracking error. The PSDs of Fig. 5.21(b) clearly show the reduction of the disturbances present in the feedforward signals obtained with wavelet filtering. 


\subsection{Discussion}

The wavelet filtering methods are tested on three different kinds of systems, a flexible shaft system, which is an academic system with especially designed dynamic properties, a relatively simple industrial system in the form of a printer and a complex industrial system, a robot arm called the pizzabot.

The wavelet filtering methods make it possible to learn up to a larger frequency where the non-repetitive part of the error becomes dominant. Without wavelet filtering, the learned feedforward signal is dominated by the disturbances. With wavelet filtering, a feedforward signal with significantly less disturbances is obtained together with a smaller tracking error. The mean rms values of the error after convergence are shown in Table 5.1 for the various wavelet filtering methods and for all three systems. It can be seen that the experiments with wavelet filtering lead to smaller tracking errors than without wavelet filtering. The improvements made by the wavelet filtering methods are not only at high frequencies but in the entire frequency range. The differences between the errors obtained with wavelet filtering are very small.

Table 5.1: Mean rms values of error after convergence

\begin{tabular}{c|c|c|c}
\multirow{2}{*}{$\begin{array}{c}\text { Filtering } \\
\text { method }\end{array}$} & \multicolumn{3}{|c}{ Mean error rms value after convergence } \\
\cline { 2 - 4 } & flexible shaft $[\mathrm{rad}]$ & printer $[$ counts] & pizzabot $[\mathrm{rad}]$ \\
\hline No filtering & $6.0533 \cdot 10^{-3}$ & 7.9596 & $6.1226 \cdot 10^{-4}$ \\
2 run & $3.4486 \cdot 10^{-3}$ & 2.8836 & $3.6899 \cdot 10^{-4}$ \\
Recursive (RE) & $4.2688 \cdot 10^{-3}$ & 4.5369 & $4.2831 \cdot 10^{-4}$ \\
Higher order (HO) & $4.3044 \cdot 10^{-3}$ & 3.5112 & $3.7879 \cdot 10^{-4}$
\end{tabular}

The recursive method does not give good results on either of the setups. This is because the linear model of the process sensitivity $S_{P}$ is not able to describe the system behavior well enough such that the measured and reconstructed error signal can be compared successfully. The error is completely removed by the wavelet filtering and no feedforward update is performed. The next iteration, the reconstructed error is only composed of the measured error signal of the previous iteration. Since no feedforward update was performed, the two error signals have an equal repetitive part. With this, the amount of runs needed for convergence is doubled and the recursive method degrades in performance to the two run method.

The higher order method shows some improvements in the amount of disturbances present in the learned feedforward signals. The small improvements do not lead to smaller tracking errors, making the higher order method not that beneficial compared to the additional runs required. However, note that the higher order method is not fully exploited.

For ILC to be successful, the systems should start at the same position every run, which is achieved by using accurate homing procedures. The learning filters derived by ZPETC [36] have an amplitude distortion at frequencies around half the sample frequency. If this frequency is close to resonance frequencies, the repetitive errors at frequencies around the resonance frequencies will not be learned correctly. This leads to errors which are dominated by erroneously learned repetitive parts. In this case no benefit can be obtained by applying wavelet filtering. The sampling frequency should be high enough to prevent an amplitude distortion of the learning filter around resonance frequencies.

The performance of the wavelet filtering methods depends on the choice of the constant part of the threshold $\gamma_{c}$. The constant part of the threshold $\gamma_{c}$ has to be tuned manually. A too large $\gamma_{c}$ will cause non-repetitive disturbances to appear in the reconstructed error signal and results in an increase of the tracking error after convergence. A too small $\gamma_{c}$ will also remove repetitive parts of the error in the reconstruction, this also leads to an increase of the tracking error after convergence. 


\section{Chapter 6}

\section{Conclusions and recommendations}

Iterative learning control can derive a high performance feedforward signal for systems that perform repetitive tasks. With the feedforward signal, the tracking performance of the system can be improved significantly. The tracking error typically consists of two parts, a repetitive part which is equal every iteration and a non-repetitive part, i.e. noise and other disturbances. ILC only compensates for the repetitive part of the error.

In this thesis, the influence of the non-repetitive disturbances on the learning scheme of ILC is investigated. Filtering methods are designed which remove the non-repetitive part of the error and lead to an improved ILC performance. The filtered error is used as input for ILC. The wavelet filtering methods are based on the discrete wavelet transform (DWT) which makes it possible to adjust the momentary frequency content of the error.

Three different wavelet filtering methods are designed. All methods are based on the same principle of calculating a measure of similarity and thresholding of the coefficients of the similarity measure. The threshold consists of two parts, a constant part and da variable part which changes based on the mean power of the wavelet coefficients in the particular frequency band. Using the adjusted wavelet coefficients, an error signal is reconstructed which ideally contains only the repetitive part of the error.

The wavelet filtering methods differ in the amount of runs which have to be performed at each iteration of ILC. The two run method uses two runs each iteration. The recursive method performs one run each iteration. The second error signal is constructed from the error of the previous iteration and the feedforward update, filtered by a model of the process sensitivity. The higher order method uses four runs each iteration and adjusts the wavelet coefficients based on two similarity criteria.

\subsection{Conclusions}

An expression for the tracking error has been derived which shows the influence of non-repetitive disturbances on the tracking error. The disturbances of the last two iterations determine the tracking error to a great extend, whereas older disturbance signals only have a limited influence on the tracking error. The non-repetitive disturbances are also present in the learned feedforward signal and deteriorate the performance of ILC. Model uncertainty also plays a part in the tracking error and convergence speed of ILC.

In order to identify the non-repetitive part of the error, a similarity criterion is developed. The similarity is evaluated based on a relative error between sets of wavelet coefficients. The coefficients of the similarity criterion are thresholded by a threshold level which consists of a constant and a variable part. The similarity is evaluated based on a relative error between sets of wavelet coefficients. The quality of the identification depends to a great extend on the threshold level. The constant part of the threshold has to be tuned manually. However, this can be done fairly easy. 
The non-repetitive errors are not completely removed by the wavelet filtering methods. Wavelet coefficients of non-repetitive errors with an amplitude smaller than the threshold level are not removed. Furthermore, with the non-repetitive disturbances also some part of the repetitive error, at equal time instants and frequency bands, is removed.

The simulations and experiments show that the wavelet filtering methods are all able to remove the repetitive part of the error. With the wavelet filtering, the errors converge to smaller values then without wavelet filtering. Furthermore, the learned feedforward signals with wavelet filtering contain significantly less disturbances. The achieved error reduction of all three wavelet filtering methods is nearly equal. The performance of the recursive method depends to a great extend on the quality of the model of the process sensitivity. The learned feedforward of the recursive method contains a larger part of the disturbances because of the differences between the model and the real process sensitivity $S_{P}$. In the experiments, the filtering with the linear model of the process sensitivity does not reconstruct the error accurate enough, degrading the performance of the recursive method to the two run method. The higher order method improves the learned feedforward signals in the sense that less disturbances are present, however the error after convergence is of equal size as for the other two methods. The higher order method is computationally more demanding and it takes more time to perform the simulations and experiments.

The wavelet filtering methods make it possible to learn up to a higher frequency. When learning up to high frequencies where the non-repetitive part of the error becomes dominant, the classic ILC scheme has a deteriorated performance and the learned feedforward is dominated by noise. With wavelet filtering, a feedforward signal is learned which contains almost no noise and the tracking error converges to a smaller value.

\subsection{Recommendations}

As a possible continuation and extension of this project, the following recommendations for further research are given:

- The recursive method does not give satisfactory results in the experiments. The linear model of the process sensitivity does not reconstruct the error signal accurate enough. For example, the learned feedforward compensates the friction present in the system. The friction is however not included in the linear model. The experimental performance of the recursive method can be improved by using a more detailed model. Instead of linear filtering, a simulation can be performed or an observer can be used.

- Construct a recursive formulation for the higher order method. This combines the advantages of the higher order method and the recursive method, i.e. less disturbances present in the feedforward without having to perform multiple runs every iteration. Note that the problems regarding the recursive method have to be solved first.

- The identification of the non-repetitive errors is done by calculating a similarity criterion using two sets of wavelet coefficients. The similarity criterion, derived in this thesis, is a relative error between these two sets. The wavelet coefficients can also be used in a different manner to identify the non-repetitive part of the error, e.g. by different similarity criteria.

- The higher order method shows some improvements in the identification of the non-repetitive disturbances. However, only two criteria are calculated using the four error signals. By further exploitation of the higher order method the identification of the non-repetitive errors can perhaps be improved.

- In this thesis, wavelet filtering is used to remove the non-repetitive part of the error. Some alternative methods can possibly also be used to eliminate the non-repetitive part and result in a disturbance free error signal. These alternative methods include using an observer and independent component analysis. Both methods will be shortly addressed. 
- An output observer uses the measured output and the output of a model to estimate an optimal output. The measured output contains both the repetitive part and the disturbances. The model output contains only the repetitive part, but the output can be non-perfect due to model errors. The observer compensates the model errors and removes the disturbances, leading to an optimal output signal.

The control output, consisting of the output of the feedback controller and the learned feedforward can be applied to a model of the system and the system itself. The outcome of the model of the system and the measured system output can be used in an observer $[15,27,18]$. The outcome of the observer can be used in the feedback control loop, see Fig. 6.1.

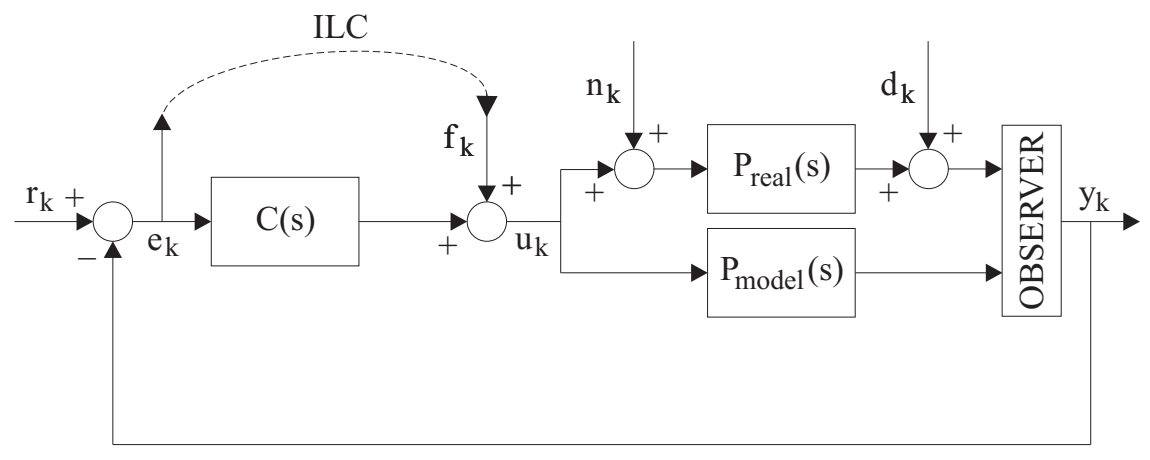

Figure 6.1: Block scheme with output observer

The idea is that the observer uses both the model output and the measured system output to obtain an improved output which contains only the repetitive part of the error and no disturbances. If the observer output is used as shown in Fig. 6.1, the error and with this the learned feedforward of ILC should also be free of disturbances.

- In [7, 19], the Independent Component Analysis (ICA) concept is presented. ICA is also known as Blind Source Separation (BSS). The goal of ICA is the decomposition of a set of data in an a priory unknown linear mixture of a priori unknown source signals [7]. The method relies on the assumption that the source signals are mutually statistically independent [16].

For application in ILC, three sources have to be separated, i.e. the repetitive part of the error, the load disturbances and the measurement disturbances. For this, ICA needs three signals which contain different linear combinations of the three signals to be separated. These signals are the tracking error, the output of the controller and the input of the system, i.e. after the summation with the load disturbances. A Matlab toolbox which incorporates ICA can be found in [16]. 


\section{Bibliography}

[1] F. Abramovich and Y. Benjamini. Adaptive thresholding of wavelet coefficients. Technical report, Department of Statistics and Operations Research, Tel Aviv University, 1999.

[2] S. Arimoto, S. Kawamura, and F. Miyazaki. Better operation of robots by learning. Journal of Robotic Systems, 1(2):123-140, 1984.

[3] O.H. Bosgra and M. Steinbuch. Lifted approach to ILC/repetitive control. Technical report, Dutch Institute of Systems and Control (DISC), 2003/2004.

[4] Y. Chen and K.L. Moore. Harnessing the nonrepetitiveness in iterative learning control. IEEE Conference on Decision and Control, pages 3350-3355, December 2002.

[5] H.J.L.M. Consten. Design of an iterative learning controller. Trainee report, Eindhoven University of Technology, July 2003.

[6] P.F. Craigmile and D.B. Percival. Wavelet-based trend detection and estimation. Technical report, University of Washington, Seattle, December 2000.

[7] L. de Lathauwer, B. de Moor, and J. Vandewalle. An introduction to independent component analysis. Journal of Chemometrics, 14(3):123-149, June 2000.

[8] D. de Roover. ZPETC.m. Matlab function, January 1995. Zero phase error tracking control algorithm implemented in Matlab.

[9] B.G. Dijkstra. Iterative Learning Control with applications to a wafer-stage. PhD thesis, Delft University of Technology, September 2004.

[10] B.G. Dijkstra and O.H. Bosgra. Extrapolation of optimal lifted system ILC solution, with application to a waferstage. Proceedings of the ACC, pages 2595-2600, May 2002.

[11] B.G. Dijkstra and O.H. Bosgra. Noise suppression in buffer-state iterative learning control, applied to a high precision wafer stage. IEEE International Conference on Control Applications, pages 998-1003, September 2002.

[12] D.L. Donoho and I.M. Johnstone. Threshold selection for wavelet shrinkage of noisy data. Proceedings of the 16th Annual International Conference of the IEEE, 1:A24-A25, 1994.

[13] R.M.L. Ellenbroek. Time-frequency adaptive iterative learning control. Master's thesis, Eindhoven University of Technology, February 2003.

[14] D.A. Francis and W.M. Wonham. The internal model principle for linear multivariable regulators. Applied Mathematics and Optimization, 2:170-194, 1975.

[15] N. Hovakimyan, F. Nardi, and A.J. Calise. A novel error observer based adaptive output feedback approach for control of uncertain systems. IEEE transactions on automatic control, 47(8):1310-1314, August 2002. 
[16] J. Hurri, H. Gavert, J Sarela, and A Hyvarinen. The fastICA package for MATLAB. Matlab toolbox, July 2004. URL: http://www.cis.hut.fi/projects/ica/fastica.

[17] S. Kivikunnas. Overview of process trend analysis methods and applications. Technical report, University of Oulu, Department of Process Engineering, August 1999.

[18] A. Kutay, A. Calise, and N. Hovakimyan. Adaptive output feedback control with reduced sensitivity to sensor noise. Proceedings of the American Control Conference, 6:5141-5146, June 2003.

[19] T. Lee. Independent Component Analysis, theory and applications. Kluwer Academic Publishers, 3 edition, 2001. ISBN: 0-7923-8261-7.

[20] R.J.E. Merry. Wavelet theory and applications, a literature study. Master's thesis, Eindhoven University of Technology, June 2005.

[21] R.J.E. Merry and M.J.G. van de Molengraft. Iterative learning control with wavelet filtering. Abstract accepted for the $24^{\text {th }}$ Benelux meeting on Systems and Control, March 2005.

[22] R.J.E. Merry, M.J.G. van de Molengraft, and M. Steinbuch. Disturbances and model uncertainties in iterative learning control. $4^{\text {th }}$ International workshop on Multidimensional Systems (NDS), July 2005.

[23] K.L. Moore. An iterative learning control algorithm for systems with measurement noise. IEEE Conference on Decision and Control, pages 270-275, December 1999.

[24] M. Norrlöf and S. Gunnarson. Some results on iterative learning control with disturbances. Technical report, Department of Electrical Engineering, Linköping University, Sweden, 1998.

[25] M. Norrlöf and S. Gunnarson. Disturbance aspects of iterative learning control. Engineering Applications of Artificial Intelligence, Elsevier, 14:87-94, 2001.

[26] S. Panzieri and G. Ulivi. Disturbance rejection of iterative learning control applied to trajectory tracking for a flexible manipulator. Proceedings of 3rd European Control Conference, pages 2374-2379, September 1995.

[27] J.H. Park and S.H. Kim. Direct adaptive output-feedback fuzzy controller for a nonaffine nonlinear system. IEEE proceedings - control theory and applications, 151(1):65-72, January 2004.

[28] I. Rotariu, R. Ellenbroek, and M. Steinbuch. Time-frequency analysis of a motion system with learning control. American Control Conference, pages 3650-3654, June 2003.

[29] M.G.E. Schneiders. Wavelets in control engineering. Master's thesis, Eindhoven University of Technology, August 2001.

[30] M. Steinbuch and M.J.G. van de Molengraft. Iterative learning control of industrial motion systems. Proc. 1st IFAC conference on Mechatronic Systems, pages 967-972, 2000.

[31] G. Strang and T. Nguyen. Wavelets and Filter Banks. Wellesley-Cambridge Press, second edition, 1997. ISBN 0-9614088-7-1.

[32] Z.R. Struzik and A. Siebes. The haar wavelet transform in the time series similarity paradigm. Technical report, Center for Mathematics and Computer Science, October 1999.

[33] W. Sun, A. Palazog̃lu, and J.A. Romagnoli. Detecting abnormal process trends by waveletdomain hidden markov models. American Institute of Chemical Engineers Journal, 49(1), 2003. 
[34] M.P. Tarvainen, P.O. Ranta-aho, and P.A. Karjalainen. An advanced detrending method with application to HRV analysis. IEEE Transactions on Biomedical Engineering, 49(2):172-175, February 2002.

[35] R.B.G. Tinsel. Iterative learning control for a gantry type manipulator. Master's thesis, University of Twente, Department of Mechanical Engineering, April 2001.

[36] M. Tomizuka. Zero phase error tracking algorithm for digital control. ASME Journal of Dynamic Systems, Measurement and Control, 109(1):65-68, March 1987.

[37] R. Tousain, E. van der Merché, and O. Bosgra. Design strategies for iterative learning control based on optimal control. IEEE Conference on Decision and Control, pages 4463-4468, December 2001.

[38] M. Uchiyama. Formulation of high-speed motion pattern of a mechanical arm by trial. Transactions of the SICE, 14(6):706-712, 1978. (in Japanese).

[39] Y. Wu, D. Agrawal, and A.E. Abbadi. A comparison of DFT and DWT based similarity search in time series databases. Proceedings of the Conference on Information and Knowledge Management, pages 488-495, 2000.

[40] Y. Ye and D. Wang. Better robot tracking accuracy with phase lead compensated ILC. IEEE International Conference on Robotics and Automation, pages 4380-4385, September 2003.

[41] B. Zhang, D. Wang, and Y. Ye. Wavelet transform-based frequency tuning ILC. IEEE Transactions on systems, man and Cybernetics - Part B, 35(1), 2005.

[42] D. Zheng and A. Alleyne. Stability of a novel iterative learning control scheme with adaptive filtering. Proceedings of the ACC, pages 4512-4517, June 2003. 


\section{Appendix A}

\section{Zero Phase Error Tracking Control}

The zero phase error tracking algorithm (ZPETC) [36] calculates an inverse of a transfer function. An exact inverse can become unstable in case the original transfer function contains non-minimumphase zeros. The ZPETC algorithm applies pole/zero cancelation to the cancelable zeros and phase cancelation to the uncancelable zeros [5] and is designed for discrete filters. Given a discrete transfer function $H\left(z^{-1}\right)$ which consists of a $d$-step delay of $z^{-d}$ and $B\left(z^{-1}\right)=b_{0}+b_{1} z^{-1}+\ldots+$ $b_{m} z^{-m}, b_{0} \neq 0$ and $A\left(z^{-1}\right)=a_{0}+a_{1} z^{-1}+\ldots+a_{n} z^{-n}, a_{0} \neq 0$

$$
H\left(z^{-1}\right)=z^{-d} \frac{B\left(z^{-1}\right)}{A\left(z^{-1}\right)} .
$$

The ZPETC algorithm calculates the inverse in several steps, the first step splits the numerator in an acceptable stable part $B^{a}\left(z^{-1}\right)$ and an unacceptable part $B^{u}\left(z^{-1}\right)$

$$
H\left(z^{-1}\right)=z^{-d} \frac{B^{a}\left(z^{-1}\right) B^{u}\left(z^{-1}\right)}{A\left(z^{-1}\right)} .
$$

If the order of $B^{a}\left(z^{-1}\right)$ equals $a$ and the order of $B^{u}\left(z^{-1}\right)$ equals $u$, the number of non-invertible zero's equals $u$. $B^{u}\left(z^{-1}\right)$ contains the zeros of $H\left(z^{-1}\right)$ which are located outside the unit circle, i.e. the non-minimum zeros.

The invertible part is inverted by the algorithm. The non-invertible part is used to scale the steady state gain, denoted by the term $B^{u}(1)$ in (A.3). The inverse transfer function is calculated by:

$$
H^{-1}\left(z^{-1}\right)=\frac{A\left(z^{-1}\right) B^{u^{*}}\left(z^{-1}\right)}{B^{a}\left(z^{-1}\right)\left(B^{u}(1)\right)^{2}}
$$

where

$$
B^{u^{*}}\left(z^{-1}\right)=b_{s}^{u}+b_{s-1}^{u} z^{-1}+\ldots+b_{0}^{u} z^{-s}
$$

The extra term in the numerator $B^{u^{*}}\left(z^{-1}\right)$ of order $u$ introduces an extra delay $u$, the total delay now becomes $d+u$. A more detailed description of the ZPETC algorithm can be found in [36, 5].

From (A.3) it follows that no phase error is made by the ZPETC algorithm. However the filter does cause an amplitude error. The amplitude match is especially bad in the high frequency region because this is where the unstable (non-minimum phase) zeros are usually located [5].

The ZPETC method makes the inverse proper by adding additional poles or zeros at high frequencies $[5,36]$. The additional poles or zeros are necessary to have a zero phase error at high frequencies. 
In zpetc.m [8] the length of the numerator and denominator are made equal by the routine tfchk.m. The unstable zeros of the original transfer function are modified to zeros in the inverse $\left(\right.$ zero $_{i n v}=1 /$ zero $\left._{t f}\right)$. This results in a phase shift because what should be a pole is constructed as a zero, additional poles are needed to correct the phase. These additional poles are located at high frequencies to make the amplitude error as small as possible.

Table A.1 shows the zpk (zero, phase and gain) data of the discrete process sensitivity calculated for the fourth order system of the simulations of Chapter 4 . It can be seen that the $S_{P}$ contains one non-minimum-phase zero. This zero also becomes a zero in the $L$ filter. The additional zero in the $L$ filter invokes the need for two additional poles in order to correct the phase of the inverse. The two additional poles are added at high frequencies, which can be seen by the two zeros in the poles of Table A.2.

\begin{tabular}{c|c|c}
$\mathrm{z}$ & $\mathrm{p}$ & $\mathrm{k}$ \\
\hline 0.9883 & $0.9969+0.0371 \mathrm{i}$ & $1.2018 \cdot 10^{-6}$ \\
0.7582 & $0.9969-0.0371 \mathrm{i}$ & \\
-1.0001 & $0.9967+0.0007 \mathrm{i}$ & \\
$-0.9999+0.0001 \mathrm{i}$ & $0.9967-0.0007 \mathrm{i}$ & \\
$-0.9999+0.0001 \mathrm{i}$ & 0.9945 &
\end{tabular}

Table A.1: zpk notation of the discrete process sensitivity $S_{P}$

\begin{tabular}{c|c|c}
$\mathrm{z}$ & $\mathrm{p}$ & $\mathrm{k}$ \\
\hline-0.9999 & 0 & $2.0803 \cdot 10^{5}$ \\
$0.9969+0.0371 \mathrm{i}$ & 0 & \\
$0.9969-0.0371 \mathrm{i}$ & $-0.9999+0.0001 \mathrm{i}$ & \\
$0.9967+0.0007 \mathrm{i}$ & $-0.9999-0.0001 \mathrm{i}$ & \\
$0.9967-0.0007 \mathrm{i}$ & 0.9883 & \\
0.9945 & 0.7582 &
\end{tabular}

Table A.2: zpk notation of the learning filter $L$ 


\section{Appendix B}

\section{Iterative learning control with wavelet filtering}

Abstract accepted for the $24^{\text {th }}$ Benelux meeting on Systems and Control: March 22-25, 2005, Houffalize, Belgium

"Iterative learning control with wavelet filtering"

The authors of this abstract are

Roel Merry

René van de Molengraft 



\section{Iterative learning control with wavelet filtering}

\author{
R.J.E. Merry \\ Eindhoven University of Technology \\ Department of Mechanical Engineering \\ Control Systems Technology Group \\ P.O. Box 513, 5600 MB Eindhoven \\ The Netherlands \\ Email: R.J.E.Merry@student.tue.nl
}

\author{
M.J.G. v.d. Molengraft \\ Eindhoven University of Technology \\ Department of Mechanical Engineering \\ Control Systems Technology Group \\ P.O. Box 513, 5600 MB Eindhoven \\ The Netherlands \\ Email: M.J.G.v.d.Molengraft@tue.nl
}

\section{Introduction}

Iterative Learning Control (ILC) is an efficient method to derive a feedforward signal for systems which follow the same trajectory repeatedly. Two kind of errors occur when following a trajectory; repetitive and non-repetitive errors. The derived feedforward signal only compensates for the repetitive part of the error. The non-repetitive errors deteriorate the performance of ILC [1].

The goal of the research is to develop a wavelet-based filtering method to filter out the non-repetitive part of the error signal and with this improve the tracking performance of ILC.

\section{Disturbances in ILC}

When ILC is applied to a system $P$ with feedback controller $C$, the learning filter $L$ is based on a model of the process sensitivity $S_{P}=P /(1+P C)$. Robustness against modeling errors is obtained by means of a filter $Q$. The feedforward update $f_{k+1}$ is calculated from the error signal $e_{k}$ as:

$$
f_{k+1}=Q\left(f_{k}+L e_{k}\right)
$$

The tracking error at iteration $k$ for a closed loop system with input disturbance $n_{k}$ and output disturbance $d_{k}$ can be formulated, with the sensitivity $S=1 /(1+P C)$ and the reference trajectory $r$, as:

$$
e_{k}=S r-S d_{k}-S_{P} n_{k}-S_{P} f_{k}
$$

Using Eqs. 1 and 2, the tracking error of iteration $k$ can be calculated as a function of $r, n_{k}$ and $d_{k}$. The resulting error equation depends on the disturbance signals of the current and the previous iterations. It can be shown that the disturbance signals of the last two iterations have the largest influence on the tracking error.

\section{Wavelet filtering}

Whereas in [2] the feedforward signal of ILC is filtered using a time-frequency analysis, this research focusses on the tracking error directly using a Discrete Wavelet Transform (DWT). The DWT uses an analysis wavelet filter bank to decompose the error signal into wavelet coefficients at various frequency bands [3].

The wavelet coefficients of the repetitive part of the error are equal for error signals of subsequent experiments, whereas the non-repetitive part results in different wavelet coefficients. The resemblance between the coefficients of various error signals is calculated using an error criterion. The coefficients which not do meet the error criterion are adjusted.

The adjusted wavelet coefficients are used in a synthesis wavelet filter bank to construct a filtered error signal. The reconstructed error signal is used as input for the learning algorithm of ILC (see Fig. 1).

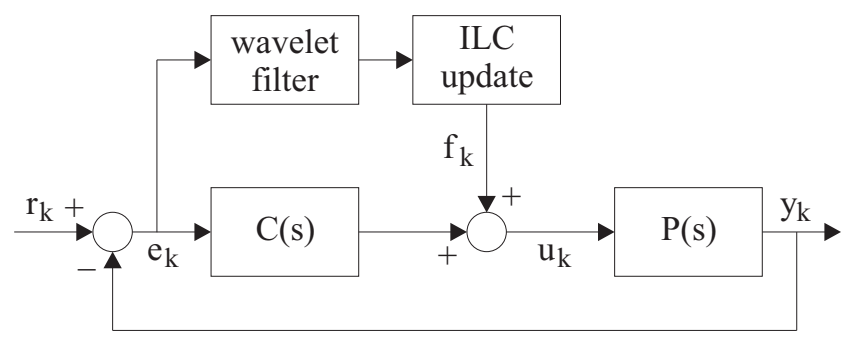

Figure 1: ILC scheme with wavelet filtering

The presentation will discuss the state of affairs in the project.

\section{References}

[1] M. Norrlöf and S. Gunnarson. Disturbance aspects of iterative learning control. Engineering Applications of Artificial Intelligence, Elsevier, 14:87-94, 2001.

[2] A.I. Rotariu, R.M.L. Ellenbroek and M. Steinbuch. Time-frequency analysis of a motion system with learning control. Proceedings of the American Control Conference, June, 2003.

[3] G. Strang and T. Nguyen. Wavelets and Filter Banks. Wellesley-Cambridge Press, second edition, 1997. 


\section{Appendix C}

\section{The influence of disturbances in iterative learning control}

Paper submitted for the IEEE Conference on Control Applications (CCA): August 28-31, 2005, Toronto, Canada

"The influence of disturbances in iterative learning control"

The authors of this paper are

Roel Merry

René van de Molengraft

Maarten Steinbuch 



\title{
The influence of disturbances in iterative learning control
}

\author{
Roel Merry, René van de Molengraft and Maarten Steinbuch
}

\begin{abstract}
For systems that perform repetitive tasks a high performance feedforward signal can be derived using Iterative Learning Control (ILC). The feedforward signal is updated through successive iterations. Disturbances present in the control scheme, such as load and measurement disturbances, are also present in the learning process and deteriorate the performance of ILC. This paper presents an expression for the tracking error of an arbitrary iteration which shows the influence of measurement and load disturbances of the present and previous iterations. The expression is validated by means of simulations and experiments on a motion system. The disturbances of the last two iterations prove to have the largest influence on the tracking error.
\end{abstract}

\section{INTRODUCTION}

Iterative Learning Control (ILC) is a powerful method to derive a high performance feedforward signal for systems that perform the same task repeatedly, even when little knowledge about the system is available. The feedforward signal is updated through successive iterations. When performing a motion task, two kind of errors occur: repetitive errors that are identical every iteration and non-repetitive errors that vary every iteration, e.g. disturbances. ILC can only compensate for the repetitive part of the error. The nonrepetitive errors also enter the learning process and limit the achievable performance. The focus of this paper is to show how non-repetitive load and measurement disturbances affect the tracking error and learning process of ILC for a system with feedback controller.

Some research on the influence of disturbances on ILC is already performed in the past. A disturbance analysis is performed in [4] for the open-loop case and measurement disturbances only. In [2] a feedback loop is included in the analysis. The performance of the learning algorithm is evaluated by the output of the feedback controller for the case of no measurement disturbances. In [3] a recursive formulation of the tracking error is used in combination with a disturbance free error signal obtained without any ILC input. Furthermore the load disturbances are assumed repetitive over the iterations. In this paper, we will provide a rigorous treatment of disturbance effects for a closedloop system and give a non-recursive expression of the tracking error of ILC in which the influence of both the load and measurement disturbances of all iterations can directly be evaluated. The error expression is validated for both sinusoidal and stochastic disturbances.

R.J.E. Merry, M.J.G. van de Molengraft and M. Steinbuch are with the Faculty of Mechanical Engineering, Control Systems Technology group, Eindhoven University of Technology, 5600 MB Eindhoven, The Netherlands, r.j.e.merry@student.tue.nl, http://www.dct.tue.nl

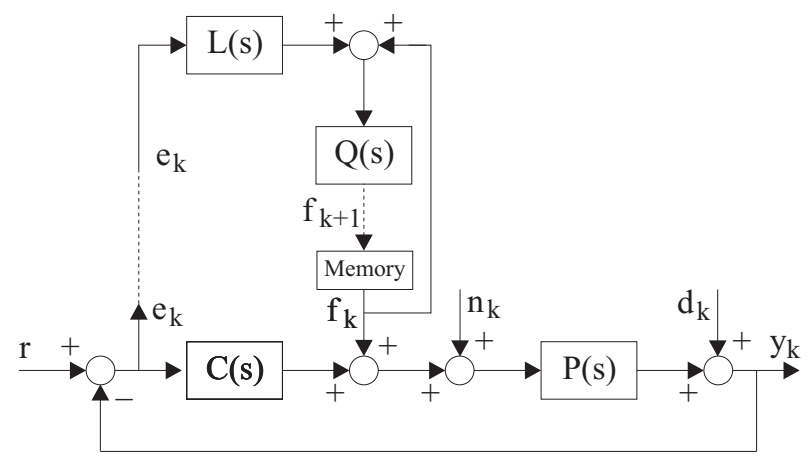

Fig. 1. Block diagram of a system with feedback controller and ILC

This paper is organized as follows. In Section II the ILC concept is explained in more detail. The influence of disturbances on the tracking error of ILC is investigated in Section III and an expression for the tracking error is derived. In Section IV the effect of harmonic load and measurement disturbances is validated by means of both simulations and experiments using a motion system. The main results are summarized in Section V.

\section{ITERATIVE LEARNING CONTROL}

A block diagram of a system $P(s)$ with feedback controller $C(s)$ and ILC added is shown in Fig. 1. The tracking error $e(t)$ is defined as the difference between the reference signal $r(t)$ and the measured position $y(t)$. The feedforward signal of ILC is denoted by $f(t)$. The signals $d(t)$ and $n(t)$ denote respectively the measurement disturbances and load disturbances. Iterations are denoted with an index, $e_{k}$ being the error of the $k^{\text {th }}$ iteration. The iterations are all of equal finite time length, $t \in\left[\begin{array}{ll}t_{0} & t_{e}\end{array}\right]$.

ILC reduces the repetitive part of the error signal during several iterations [5]. The tracking error is used off-line to calculate a feedforward signal that reduces the error from one iteration to the next. The error $e_{k}$ is filtered with a learning filter $L(s)$ and added to the feedforward $f_{k}$. The sum of $f_{k}$ and the filtered error is applied to a robustness filter $Q(s)$, resulting in the new feedforward signal $f_{k+1}$. The learning update of the feedforward signal equals

$$
f_{k+1}=Q\left(f_{k}+L e_{k}\right) .
$$

Using the closed-loop transfer from the feedforward to the error signal, i.e. the process sensitivity $S_{P}=P /(1+P C)$, the propagation of the error from iteration to iteration can be written as

$$
e_{k+1}=Q\left(1-S_{P} L\right) e_{k}
$$


The error reduces between successive iterations if

$$
\left|Q\left(1-S_{P} L\right)\right|<1
$$

From (3) follows that a suitable choice for the learning filter $L$ would be $L=S_{P}^{-1}$. In most cases, especially in motion control systems of with the position as output, the process sensitivity $S_{P}$ is strictly proper and can not be inverted. Furthermore, if $S_{P}$ contains non-minimum phase zeros, the inverse will contain unstable poles. In these cases an approximate $L \approx S_{P}^{-1}$ is determined and the $Q(s)$ filter is required to provide robustness against modeling errors and to satisfy (3). The performance of ILC is determined by the design of $L(s)$ and $Q(s)$.

\section{Disturbance ANALYSIS}

The error $e_{k}$ can be written as a function of the reference signal $r$ which is equal every iteration, the measurement disturbance $d_{k}$, the load disturbance $n_{k}$ and the feedforward $f_{k}$ as (see Fig. 1)

$$
e_{k}=S r-S d_{k}-S_{P} n_{k}-S_{P} f_{k}
$$

where the sensitivity $S=1 /(1+P C)$. The feedforward of iteration $k$ can be written with the use of (1) and $e_{k-1}$ as

$f_{k}=Q\left(1-L S_{P}\right) f_{k-1}+Q L S r-Q L S d_{k-1}-Q L S_{P} n_{k-1}$

Substitution of (5) in (4) gives

$$
\begin{array}{r}
e_{k}=-S_{P} Q\left(1-L S_{P}\right) f_{k-1}+\left(1-S_{P} Q L\right) S r-S d_{k} \\
+S_{P} Q L S d_{k-1}-S_{P} n_{k}+S_{P}^{2} Q L n_{k-1} .
\end{array}
$$

With use of (5) and (6) the error of an arbitrary iteration $k$ can be written as a function of the reference $r$ and the disturbances of the $k^{t h}$ and previous iterations by repeated substitutions as

$$
\begin{aligned}
& e_{k}=\left(1-S_{P} Q L\right) S r-\sum_{j=1}^{k-2} S_{P} Q\left[Q\left(1-L S_{P}\right)\right]^{k-j-1} L S r \\
& -S d_{k}+S_{P} Q L S d_{k-1}+\sum_{j=1}^{k-2} S_{P} Q\left[Q\left(1-L S_{P}\right)\right]^{k-j-1} L S d_{j} \\
& -S_{P} n_{k}+S_{P}^{2} Q L n_{k-1}+\sum_{j=1}^{k-2} S_{P}^{2} Q\left[Q\left(1-L S_{P}\right)\right]^{k-j-1} L n_{j}
\end{aligned}
$$

The influence of the reference $r$ on the tracking error $e_{k}$ is determined by $\left(1-S_{P} Q L\right) S$ and the first summation term of (7). The measurement and load disturbances of iteration $k$ are filtered by respectively $S$ and $S_{P}$, the disturbance signals of iteration $k-1$ by $S_{P} Q L S$ for measurement disturbances and by $S_{P}^{2} Q L$ for load disturbances. The disturbances of older iterations are filtered by the second and third summation terms of (7). The presence of the disturbances in the learning process results in a degraded performance of ILC, i.e. an increase of the tracking error.

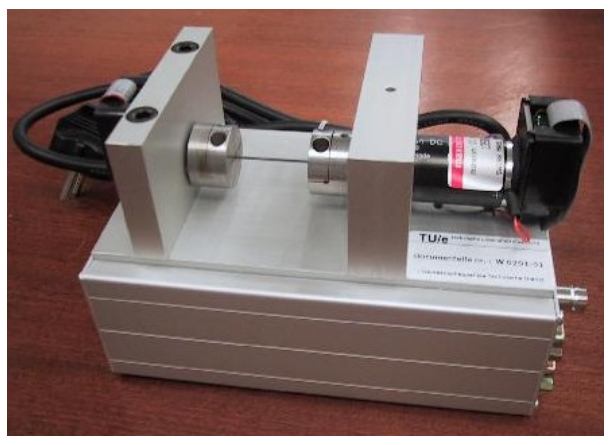

Fig. 2. System with two connected rotating masses

\section{A. Ideal learning filter}

If an ideal learning filter $L=S_{P}^{-1}$ can be derived, no $Q$ filter is needed since the convergence criterion of (3) is already met $(Q(s)=1)$. The tracking error of (7) now reduces to

$$
e_{k}=-S d_{k}+S d_{k-1}-S_{P} n_{k}+S_{P} n_{k-1} .
$$

The tracking error only depends on the disturbances of the last two iterations. In the worst case scenario the load and measurement disturbances have opposite sign with respect to the last two iterations. In case of no disturbances and an ideal learning filter the tracking error $e_{k}$ becomes zero.

\section{B. Approximation learning filter}

If $L \neq S_{P}^{-1}$, a $Q$ filter is generally needed. The error $e_{k}$ depends on the disturbances of all previous iterations. In comparison to the filters of the disturbance signals of iteration $k-1$, the filters of disturbances of older iterations have an additional multiplication with the convergence criterion. The number of multiplications depends on the iteration number, see (7). The summation terms converge to fixed transfer functions in the frequency domain for $k \rightarrow \infty$ due to the multiplication with the convergence criterion of (3) which has an amplitude smaller than one for all frequencies. The magnitudes of the Frequency Response Functions (FRFs) of the summation terms are smaller than the magnitudes of the FRFs of the disturbance filters for iterations $k$ and $k-1$. Disturbance signals of iterations older than the last two only have a small contribution to the error.

\section{VALIDATION}

The validation is performed using a system consisting of two rotating masses connected through a flexible shaft as shown in Fig. 2. The first mass is excited by a motor, the position of the second mass is measured using an incremental encoder. The measured and fitted FRF of the system are shown in Fig. 3. The fit will be used for simulation purposes and for the filter design. For the system a feedback controller is designed consisting of a lead filter and a notch, resulting in a bandwidth of the controlled system of $5 \mathrm{~Hz}$. The sampling rate of the controller equals $8 \mathrm{kHz}$.

The applicability of ILC on the system is tested by performing ten tracking experiments and determining the 


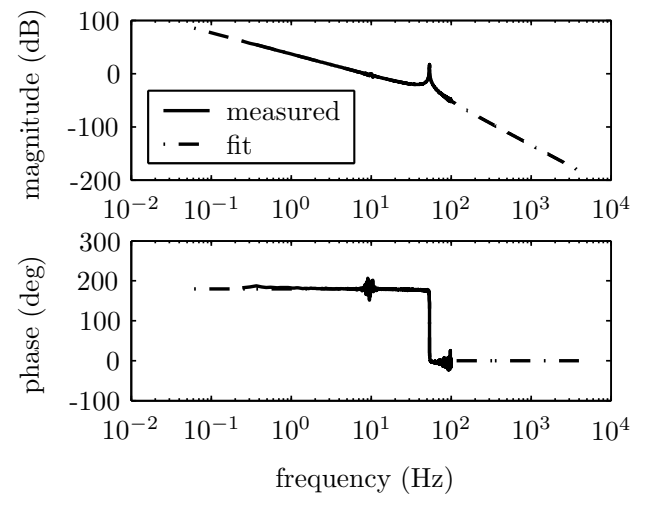

Fig. 3. FRFs system and fit

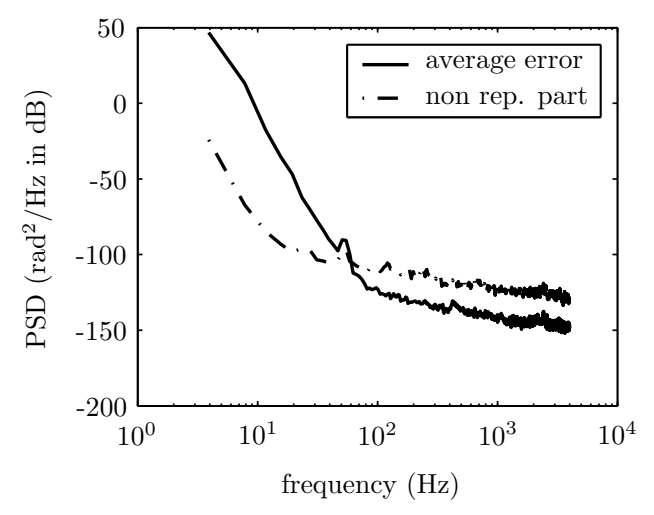

Fig. 4. PSD error signals

repetitive part of the error signals. The Power Spectral Density (PSD) of the average error, i.e. the repetitive part, is larger than the PSD of the non-repetitive part of the error up to a frequency of $60 \mathrm{~Hz}$ as shown in Fig. 4. Above this frequency the non-repetitive part dominates the error and limits the performance of ILC.

The learning filter $L$ is designed using a discrete model of $S_{P}$ and the Zero Phase Error Tracking Control (ZPETC) method proposed in [6]. The FRFs of the process sensitivity $S_{P}$ and the learning filter $L$ are shown in Fig. 5.

In order to meet the convergence criterion of (3) an eighth order low-pass $Q$ filter with a cut-off frequency of $50 \mathrm{~Hz}$ is used.

The influence of measurement and load disturbances on the tracking error of (7) is validated separately. First the performance of ILC is tested without additional disturbances. The influence of disturbances is analyzed using harmonic disturbance signals which have a constant frequency and amplitude every iteration, the initial phases $\phi_{0}$ vary every iteration.

\section{A. No disturbances}

The performance of ILC without additional disturbances is tested by means of a simulation and an experiment with 25 iterations. For the first iteration a zero feedforward is used.

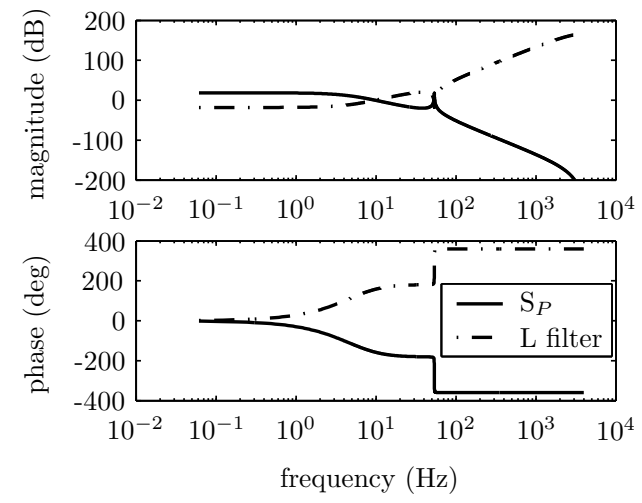

Fig. 5. FRFs $S_{P}$ and $L$ filter

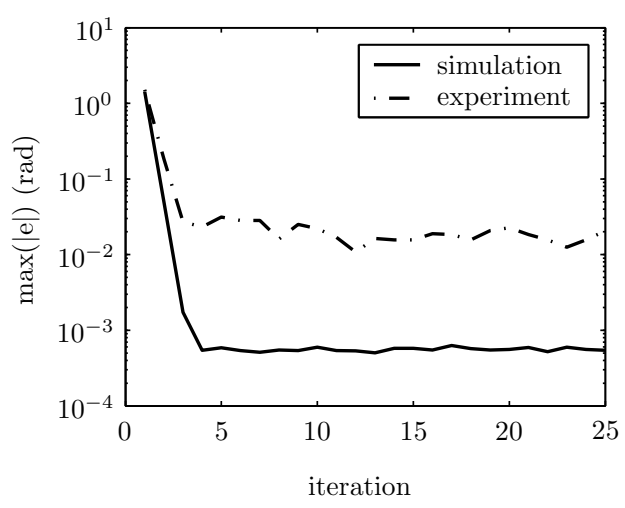

Fig. 6. Maximum absolute error without disturbances

The $L$ and $Q$ filters are equal for both the simulation and the experiment.

The maximum absolute tracking errors of the simulation and experiment without additional disturbances are shown in Fig. 6. The tracking error of the simulation converges in 4 iterations to $|e|_{\max } \approx 5 \cdot 10^{-4} \mathrm{rad}$ and the tracking error of the experiment in 8 iterations to $|e|_{\max } \approx 2 \cdot 10^{-2} \mathrm{rad}$. The fluctuation on the maximum absolute error of the simulation is due to numerical inaccuracies. The tracking error of the experiment converges to a larger value due to the presence of the non-repetitive part of the error as shown in Fig. 4.

\section{B. Measurement disturbances}

The influence of measurement disturbances $d_{k}$ on the tracking error of ILC follows from (7) as

$$
\begin{aligned}
e_{k, d}= & -S d_{k}+S_{P} Q L S d_{k-1} \\
& +\sum_{j=1}^{k-2} S_{P} Q\left[Q\left(1-L S_{P}\right)\right]^{k-j-1} L S d_{j} .
\end{aligned}
$$

The harmonic measurement disturbances are chosen such that the amplitude exceeds the magnitude of the tracking error of the ILC experiment without additional disturbance. The frequency is chosen below the cut-off frequency of the $Q$ filter. The used harmonic measurement disturbance $d_{k}$ equals

$$
d_{k}=8 \cdot 10^{-1} \sin \left(2 \pi 4 t+\phi_{0}\right) \text {. }
$$



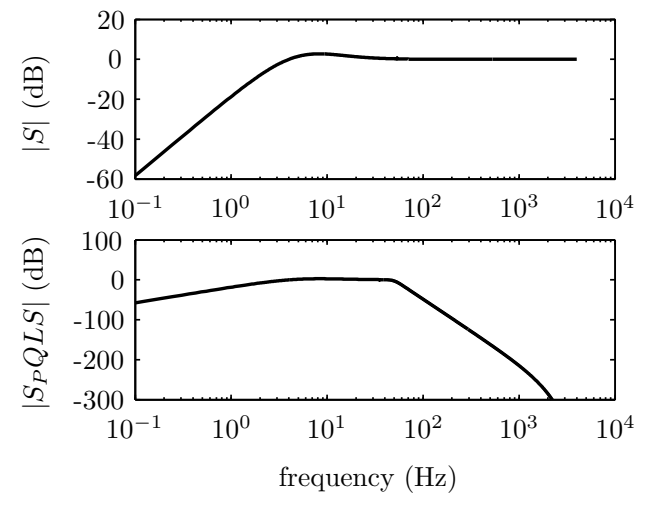

Fig. 7. Magnitudes FRFs $S$ and $S_{P} Q L S$

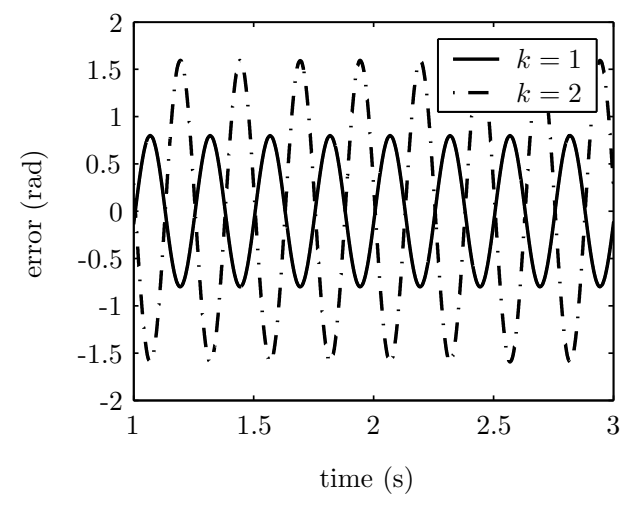

Fig. 8. Errors worst case simulation with measurement disturbances

1) Two iterations: The influence of measurement disturbances on the tracking error can be seen in (9). For an ILC simulation with two iterations the worst case error occurs if the disturbance signals have opposite sign, this corresponds to an initial phase shift of $\pi$ rad for the harmonic disturbances.

The error signal of the second iteration contains the disturbance signal of the second iteration filtered with $S$ and the disturbance signal of the first iteration filtered with $S_{P} Q L S$. The magnitudes of the FRFs of $S$ and $S_{P} Q L S$ are shown in Fig. 7.

At the disturbance frequency of $4 \mathrm{~Hz}$ the magnitudes of $|S|=0.9986$ and of $\left|S_{P} Q L S\right|=0.9905$. The maximum tracking error of the first iteration can be calculated using (7) as $\left|e_{1, d}\right|_{\max }=|S| d_{\max }=0.7989 \mathrm{rad}$. The maximum error of the second iteration equals with use of (7)

$$
\begin{aligned}
\left|e_{2, d}\right|_{\max } & =|S| d_{\max }+\left|S_{P} Q L S\right| d_{\max } \\
& =0.9986 * 8 \cdot 10^{-1}+0.9905 * 8 \cdot 10^{-1} \\
& =1.5913 \mathrm{rad} .
\end{aligned}
$$

For the simulation and experiment the initial feedforward signals equal the feedforward signals obtained from the simulation and experiment without additional disturbances of Fig. 6, making the influence of the reference $r$ negligibly small.

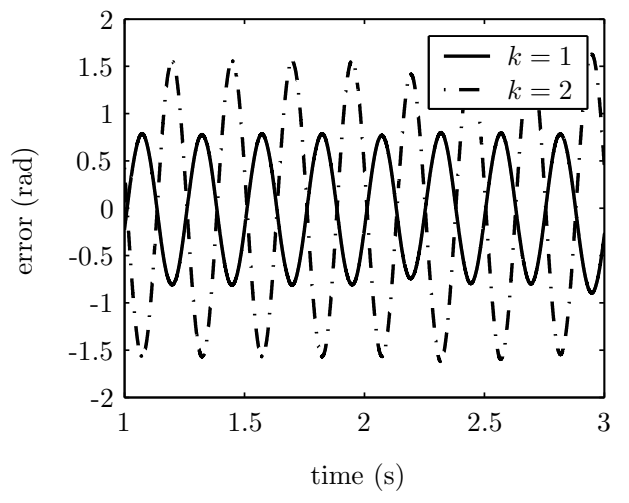

Fig. 9. Errors worst case experiment with measurement disturbances

The tracking errors of the first and second iteration for the worst case simulation and experiment with two iterations are shown in respectively Fig. 8 and Fig. 9. The amplification of the tracking error is caused by the presence of the disturbance signals in the learning process of the feedforward signal.

The calculated maximum absolute errors and the maximum absolute errors obtained from the simulation and experiment are given in Table I. The calculated maximum errors of the first and second iteration show a good correspondence with the values found in the simulation and experiment. The experimentally obtained values show a larger deviation from the calculated values as the values found in the simulation. This is caused by the magnitude of the non-repetitive error and because the learning filters are based on a fit, i.e. an approximation, of the system FRF.

2) Multiple iterations: For an ILC simulation and experiment with more than two iterations the measurement disturbances of older iterations affect the tracking error through the summation term $\sum_{j=1}^{k-2} S_{P} Q\left[Q\left(1-L S_{P}\right)\right]^{k-j-1} L S d_{j}$ in (9). From the analysis of Section III followed that the disturbances of iterations older than the last two are not likely to influence the maximum error a lot. This also follows from the ILC simulation and experiment with 25 iterations and random initial phases of the disturbance signals, shown in Fig. 10. If the random disturbance signals of the last two iterations have a very small phase difference, the contributions to the error almost cancel each other and the resulting error can become smaller than $|S| d_{\max }$. The largest maximum errors after convergence of the simulation and experiment with 25 iterations are given in Table I. The largest simulated maximum absolute error exceeds the calculated worst case error with two iterations only by $0.86 \%$, the contribution of the summation term is much smaller than the contribution of the other two terms. The experimentally obtained largest maximum absolute error exceeds the experimentally obtained maximum absolute error of the worst case experiment with two iterations only by $1.87 \%$, indicating again the limited contribution of the summation term to the total tracking error. 


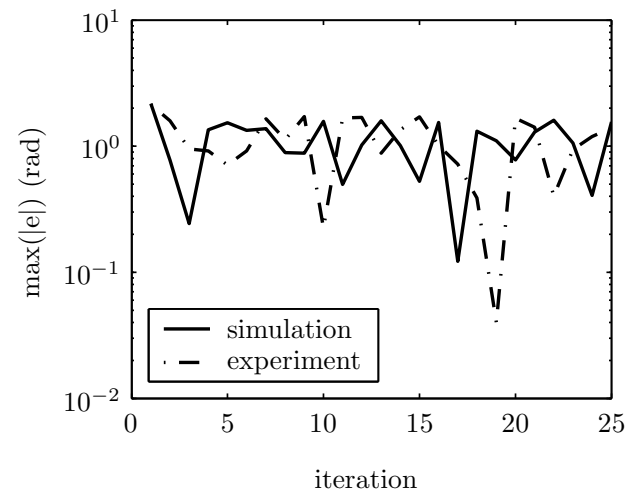

Fig. 10. Maximum absolute error with measurement disturbances

TABLE I

CALCULATED, SIMULATED AND EXPERIMENTALLY OBTAINED MAXIMUM ABSOLUTE ERRORS WITH MEASUREMENT DISTURBANCES

\begin{tabular}{c|c|c|c}
\multirow{2}{*}{ method } & \multicolumn{3}{|c}{ maximum absolute error (rad) } \\
\cline { 2 - 4 } & first iteration & second iteration & 25 iterations \\
\hline calculated & 0.7989 & 1.5913 & - \\
simulation & 0.8006 & 1.5970 & 1.6051 \\
experiment & 0.8991 & 1.6836 & 1.7151
\end{tabular}

\section{Load disturbances}

The influence of load disturbances on the tracking error results from (7)

$$
\begin{aligned}
e_{k, n}= & -S_{P} n_{k}+S_{P}^{2} Q L n_{k-1} \\
& +\sum_{j=1}^{k-2} S_{P}^{2} Q\left[Q\left(1-L S_{P}\right)\right]^{k-j-1} L n_{j} .
\end{aligned}
$$

The used harmonic load disturbance $n_{k}$ has an amplitude exceeding the maximum error obtained without additional disturbances and a frequency below the cut-off frequency of the $Q$ filter

$$
n_{k}=1 \cdot 10^{-1} \sin \left(2 \pi 1 t+\phi_{0}\right) .
$$

1) Two iterations: The effect of the load disturbances of the first two iterations on the error $e_{k}$ is given by (11), the error of the second iteration contains the disturbance signal of the second iteration filtered by $S_{P}$ and the disturbance signal of the first iteration filtered by $S_{P}^{2} Q L$. Fig. 11 shows the magnitudes of the FRFs of $S_{P}$ and $S_{P}^{2} Q L$.

As was the case for the measurement disturbances, the worst case occurs when the harmonic disturbances of the last two iterations have opposite sign, i.e. an initial phase shift of $\pi$ rad for the harmonic disturbances.

At $1 \mathrm{~Hz}$ the magnitudes are respectively $\left|S_{P}\right|=7.9636$ and $\left|S_{P}^{2} Q L\right|=8.2159$. For the first iteration the maximum absolute error equals $\left|e_{1, n}\right|_{\max }=\left|S_{P}\right| n_{\max }=0.7964 \mathrm{rad}$. The maximum absolute error of the second iteration can be calculated as

$$
\left|e_{2, n}\right|_{\max }=\left|S_{P}\right| n_{\max }+\left|S_{P}^{2} Q L\right| n_{\max }=1.6180 \mathrm{rad} .
$$
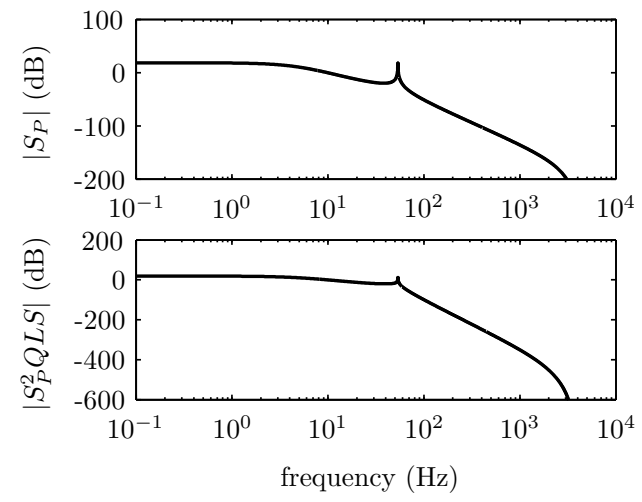

Fig. 11. Magnitudes FRFs $S_{P}$ and $S_{P}^{2} Q L$

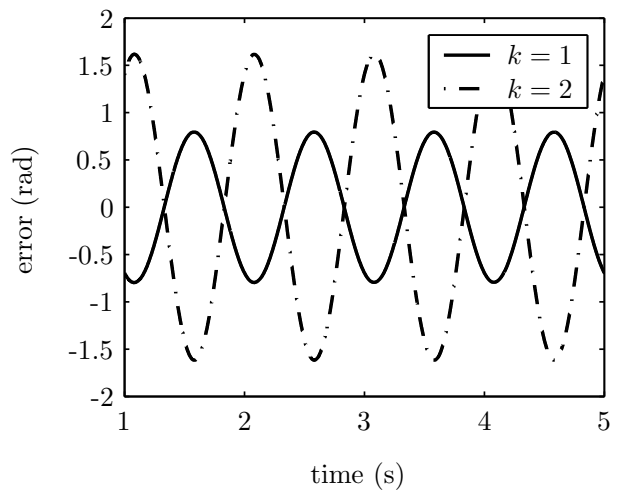

Fig. 12. Errors worst case simulation with load disturbances

The tracking errors of the two iterations of the worst case are shown in Fig. 12 for the simulation and in Fig. 13 for the experiment. The initial feedforward signals equal the feedforward signals obtained from the simulation and experiment without additional disturbances.

The maximum absolute errors of the error signals of the two iterations are given for both the simulation and experiment in Table II. The calculated values correspond to the values obtained from the simulation and experiment. The experimentally obtained values differ more because of the non-repetitive part of the error present in the experiment (see Fig. 4) and because the learning filter is based on a fit.

2) Multiple iterations: For multiple iterations, the load disturbances of the iterations older than the last two are filtered by $\sum_{j=1}^{k-2} S_{P}^{2} Q\left[Q\left(1-L S_{P}\right)\right]^{k-j-1} L n_{j}$ in (11). The disturbance signals of iterations older than the last two are not likely to have a large influence on the tracking error. This also follows from the ILC simulation and experiment with 25 iterations and a random initial phase of the disturbance signal, as can be seen in Fig. 14. Table II gives the largest maximum absolute error after convergence of the simulation and experiment with 25 iterations. The values obtained from the 25 iterations do not exceed the worst case values of 2 iterations significantly, indicating the limited contribution of the summation term. 


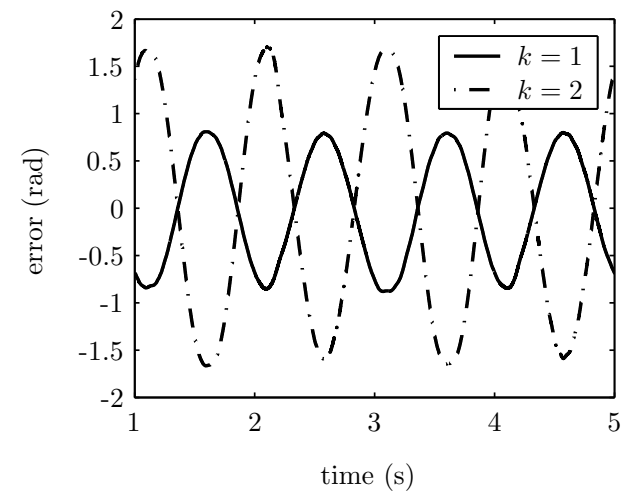

Fig. 13. Errors worst case experiment with load disturbances

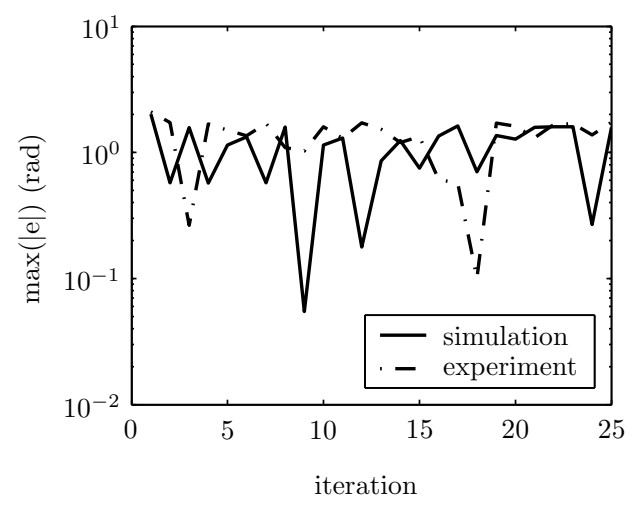

Fig. 14. Maximum absolute error with load disturbances

\section{Stochastic disturbances}

In practice the non-repetitive errors are often stochastic signals, e.g. noise. The validity of (7) for stochastic disturbances is tested by a simulation with 2 iterations and noise measurement disturbances. The noise signals have a uniform distribution in the interval $\left[-1 \cdot 10^{-2} 1 \cdot 10^{-2}\right]$. The initial feedforward equals the feedforward obtained from the simulation without additional disturbances. The maximum absolute error and root-mean-square (RMS) values of the error signals of the two iterations are given in Table III. Table III also contains the calculated values using (7). The calculated values and the values obtained by the simulation are approximately equal, indicating the validity of (7) for stochastic disturbances.

TABLE II

CAlCulated, Simulated AND EXPERIMENTALly OBTAINED MAXIMUM ABSOLUTE ERRORS WITH LOAD DISTURBANCES

\begin{tabular}{c|c|c|c}
\multirow{2}{*}{ method } & \multicolumn{3}{|c}{ maximum absolute error (rad) } \\
\cline { 2 - 4 } & first iteration & second iteration & 25 iterations \\
\hline calculated & 0.7964 & 1.6180 & - \\
simulation & 0.7972 & 1.6189 & 1.6266 \\
experiment & 0.8828 & 1.7063 & 1.7374
\end{tabular}

TABLE III

MAXIMUM ABSOLUTE VALUES AND RMS VALUES OF THE ERROR SIGNALS FOR NOISE MEASUREMENT DISTURBANCES

\begin{tabular}{c|c|c|c} 
iteration & value & calculated & simulation \\
\hline 1 & $\max \left(\left|e_{1}\right|\right)(\mathrm{rad})$ & $1.1268 \cdot 10^{-2}$ & $1.1268 \cdot 10^{-2}$ \\
1 & $R M S\left(e_{1}\right)(\mathrm{rad})$ & $5.7790 \cdot 10^{-3}$ & $5.7784 \cdot 10^{-3}$ \\
\hline 2 & $\max \left(\left|e_{2}\right|\right)(\mathrm{rad})$ & $1.7992 \cdot 10^{-2}$ & $1.8315 \cdot 10^{-2}$ \\
2 & $R M S\left(e_{2}\right)(\mathrm{rad})$ & $6.3825 \cdot 10^{-3}$ & $6.3251 \cdot 10^{-3}$
\end{tabular}

\section{CONCLusions}

An expression for the tracking error of an arbitrary iteration $k$ as function of the reference signal $r$, measurement disturbances $d_{k}$, load disturbances $n_{k}$, the system transfer function $P$, the feedback controller $C$ and learning filters $Q$ and $L$ was derived. With this expression the influence of disturbances on the tracking error can directly be evaluated. The expression is validated by means of simulations and experiments. The disturbance signals of the last two iterations determine the maximum error to a great extend. The presence of disturbances in the learning process of the feedforward signal deteriorates the performance of ILC in the sense of an amplification of the tracking error.

\section{REFERENCES}

[1] K.L. Moore. Iterative Learning Control for Deterministic Systems, Springer, Berlin, 1993.

[2] M. Norrlöf and S. Gunnarsson. Some results on iterative learning control with disturbances. Department of Electrical Engineering, Linköping University, Sweden, 1998.

[3] M. Norrlöf and S. Gunnarsson, Disturbance aspects of iterative learning control. Engineering Applications of Artificial Intelligence, Elsevier, 14:87-94, 2001.

[4] S. Panzieri and G. Ulivi. Disturbance rejection of iterative learning control applied to trajectory tracking for a flexible manipulator. Proceedings of 3rd European Control Conference, pp 2374-2379, 1995.

[5] M. Steinbuch and M.J.G. van de Molengraft. Iterative Learning Control of Industrial Motion Systems, Proc. 1st IFAC conference on Mechatronic Systems, pp 967-972, 2000.

[6] M. Tomizuka. Zero phase error tracking algorithm for digital control. ASME Journal of Dynamic Systems, Measurement and Control, 109:65-68, 1987. 


\section{Appendix D}

\section{Disturbances and model uncertainties in iterative learning control}

Paper accepted for the $4^{\text {th }}$ International workshop on Multidimensional Systems (NDS):

July 10-13, 2005, Wuppertal, Germany

"Disturbances and model uncertainties in iterative learning control"

The authors of this paper are

Roel Merry

René van de Molengraft

Maarten Steinbuch 



\title{
DISTURBANCES AND MODEL UNCERTAINTIES IN ITERATIVE LEARNING CONTROL
}

\author{
Roel Merry, René van de Molengraft and Maarten Steinbuch \\ Department of Mechanical Engineering \\ Eindhoven University of Technology \\ 5600 MB Eindhoven, The Netherlands \\ r.j.e.merry@student.tue.nl
}

\begin{abstract}
A high performance feedforward signal for systems that perform repetitive tasks can be derived using Iterative Learning Control (ILC). Disturbances present in the feedback control loop, such as measurement and load disturbances, enter the learning process and deteriorate the performance of ILC. Model uncertainties also influence the learning process and the minimum achievable error of ILC. This paper presents an expression for the tracking error of an arbitrary iteration that shows the influence of measurement disturbances, load disturbances and model uncertainties throughout the learning process. The tracking error is proven to be determined to a great extend by both the disturbances of the last two iterations and the model uncertainties.
\end{abstract}

\section{INTRODUCTION}

Iterative Learning Control (ILC) constructs a high performance feedforward signal through successive iterations for systems that perform the same task repeatedly. Two kinds of errors occur when performing a motion task: repetitive errors that are identical every iteration and non-repetitive errors that vary every iteration. ILC can only compensate for the repetitive part of the error. The non-repetitive errors, such as measurement and load disturbances, also enter the learning process and limit the achievable performance of ILC. The design of the learning filters for ILC is based on a model of the system [6], because of this the performance is also affected by model uncertainties.

The effect of non-repetitive errors and model uncertainties on ILC has already got attention in the past. An analysis for the open-loop case with measurement disturbances only is performed in [5]. In [3] a feedback loop is included. The performance is evaluated by the output of the feedback controller for the case of no measurement disturbances. A recursive formulation of the tracking error is presented in the analysis of [4] together with a disturbance free error signal obtained without any ILC input. Furthermore the load disturbances are assumed to be repetitive over the iterations. The influence of model uncertainties is investigated in [1] for the lifted ILC approach and constant model uncertainties only. The focus of this paper is to show how model uncertainties affect the tracking error of ILC for a system with feedback controller and harmonic, but non-repetitive disturbances. A non-recursive expression of the error of ILC is presented in which the influence of both measurement and load disturbances can directly be evaluated. Also, the effect of model gain uncertainties and model order uncertainties on this error expression is analyzed. The theoretical analysis is supported by simulations as well as experiments.

This paper is organized as follows. The ILC concept is explained in more detail in Section 2. The disturbance analysis and derivation of the error expression are presented in Section 3. The influence of model uncertainties on the tracking error of ILC is investigated in Section 4. Section 5 validates the theoretical analysis by means of simulations and experiments. The main results are summarized in Section 6.

\section{ITERATIVE LEARNING CONTROL}

In Fig. 1 a block diagram of a system $P(s)$ with feedback controller $C(s)$ and ILC added is shown. The tracking error $e(t)$ is defined as the difference between the reference signal $r(t)$ and the measured output $y(t)$. The measurement and load disturbances are denoted by respectively $d(t)$ and $n(t)$. The feedforward signal $f(t)$ is obtained using ILC. The iteration number is denoted by the index $k$, all iterations are of equal finite-time length, $t \in\left[\begin{array}{ll}t_{0} & t_{e}\end{array}\right]$.

ILC updates the feedforward signal off-line. The error signal $e_{k}(t)$ is stored and filtered with the learn- 


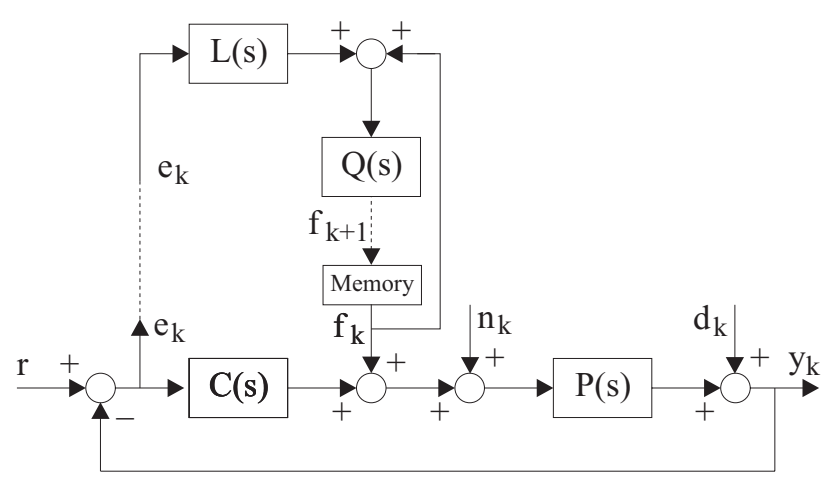

Figure 1: ILC block diagram with feedback controller

ing filter $L(s)$. The sum of the old feedforward $f_{k}(t)$ and the filtered error is filtered using a robustness filter $Q(s)$, resulting in the new feedforward $f_{k+1}$ for the next iteration.

The design of $L(s)$ and $Q(s)$ determines the performance of ILC. The ILC algorithm converges if the error $e_{k}$ reduces between subsequent iterations. The closed loop transfer from the feedforward to the error signal equals

$$
e_{k}=S_{P} f_{k},
$$

where the process sensitivity $S_{P}=P /(1+P C)$. The learning update of the feedforward signal equals

$$
f_{k+1}=Q\left(f_{k}+L e_{k}\right) .
$$

The error of iteration $k+1$ can be written as

$$
e_{k+1}=Q\left(1-S_{P} L\right) e_{k}
$$

The ILC algorithm converges if

$$
\left|Q\left(1-S_{P} L\right)\right|<1 .
$$

A suitable choice to obtain convergence is by choosing the inverse process sensitivity $S_{P}^{-1}$ for the learning filter $L$. However in most cases the process sensitivity $S_{P}$ is strictly proper and cannot be inverted. If $S_{P}$ contains non-minimum phase zeros, this will result in unstable poles in the inverse. In these cases an approximate inverse $L \approx S_{P}^{-1}$ is determined. The $Q$ filter provides robustness against modeling errors and assures that the convergence criterion (4) is satisfied.

\section{DISTURBANCE ANALYSIS}

The error signal $e_{k}$ can be written using Fig. 1 as

$$
e_{k}=S r-S d_{k}-S_{P} n_{k}-S_{P} f_{k},
$$

where the sensitivity $S=1 /(1+P C)$. The feedforward of iteration $k$ can be calculated from the error and the feedforward of iteration $k-1$ using (2) as

$$
\begin{aligned}
f_{k}=Q\left(1-L S_{P}\right) f_{k-1}+Q L S r \\
\quad-Q L S d_{k-1}-Q L S_{P} n_{k-1} .
\end{aligned}
$$

The error at iteration $k$ follows from the substitution of (6) in (5) as

$$
\begin{gathered}
e_{k}=-S_{P} Q\left(1-L S_{P}\right) f_{k-1}+\left(1-S_{P} Q L\right) S r \\
-S d_{k}+S_{P} Q L S d_{k-1}-S_{P} n_{k}+S_{P}^{2} Q L n_{k-1} .
\end{gathered}
$$

The error for an arbitrary iteration $k$ can be formulated by repeating the calculation of the feedforward signal and the error as done in (6) and (7) as

$$
\begin{gathered}
e_{k}=\left(1-S_{P} Q L\right) S r-S d_{k}-S_{P} n_{k}+S_{P} Q L S d_{k-1} \\
+S_{P}^{2} Q L n_{k-1}-\sum_{j=1}^{k-2} S_{P} Q\left[Q\left(1-L S_{P}\right)\right]^{k-j-1} L S r \\
+\sum_{j=1}^{k-2} S_{P} Q\left[Q\left(1-L S_{P}\right)\right]^{k-j-1} L S d_{j} \\
+\sum_{j=1}^{k-2} S_{P}^{2} Q\left[Q\left(1-L S_{P}\right)\right]^{k-j-1} L n_{j} .
\end{gathered}
$$

The reference $r$ contributes to the tracking error $e_{k}$ through the filter $\left(1-S_{P} Q L\right) S$ and the first summation term of (8). The reference $r$ is not varied over the iteration process. The disturbance signals present in the learning process result in an increase of the tracking error and a degraded performance of ILC.

\subsection{Ideal learning filter}

If an ideal learning filter $L=S_{P}^{-1}$ can be derived no $Q$ filter is needed to satisfy the convergence criterion of (4). The tracking error of (8) reduces with $L=S_{P}^{-1}$ and $Q(s)=1$ to

$$
e_{k}=-S d_{k}+S d_{k-1}-S_{P} n_{k}+S_{P} n_{k-1} \text {. }
$$

For an ideal learning filter the error is only determined by the disturbances of the last two iterations. The error equals zero in case of no disturbances. In the worst case the measurement and load disturbances of iteration $k$ have an opposite sign with respect to the disturbances of iteration $k-1$. 


\subsection{Approximated learning filter}

In most cases $L \approx S_{P}^{-1}$ and a robustness filter $Q$ is needed. The disturbances of all iterations contribute to the error (8). The measurement and load disturbances of iteration $k$ are filtered by respectively $S$ and $S_{P}$. The measurement and load disturbances of iteration $k-1$ appear in the error of iteration $k$ through the filters $S_{P} Q L S$ and $S_{P}^{2} Q L$. The disturbances of older iterations are filtered by the second and third summation terms of (8). In comparison with the filters of iteration $k-1$ the summation terms have an additional multiplication with the convergence criterion (4) a number of times. The number of multiplications depends on the iteration number, as can be seen in the second and third summation terms of (8).

The summation terms converge to fixed transfer functions in the frequency domain for $k \rightarrow \infty$ under the assumption that the convergence criterion of (4) is satisfied, i.e. the magnitude is smaller than one for all frequencies. The magnitudes of the Frequency Response Functions (FRFs) of the summation terms are smaller than the magnitudes of the filters for the disturbances of iteration $k-1$. The contribution of the disturbance signals older than the last two iterations is smaller than the contribution of the disturbances at iterations $k-1$ and $k$.

\section{MODEL UNCERTAINTIES}

The learning filter $L$ is derived based on a model of the process sensitivity $S_{P}$. A widely used method to obtain the model of $S_{P}$ is by using a fit of a FRF measurement of $P$ and the designed $C$. The fitted FRF of the system $P$ can contain model uncertainties. The system model $\tilde{P}$ has a model uncertainty $\Delta P$ with respect to the real system $P$ and is defined as

$$
\tilde{P}=P+\Delta P .
$$

The process sensitivity $S_{P}$ used for the design of the $L$ filter incorporates the model uncertainties as

$$
\tilde{S}_{P}=\frac{\tilde{P}}{1+\tilde{P} C} .
$$

This results in a learning filter of

$$
\tilde{L}=\tilde{S}_{P}^{-1}
$$

The influence of model uncertainties on the tracking error of ILC can be described by replacing the learning filter $L$ in (8) by the learning filter with model uncertainties $\tilde{L}$.

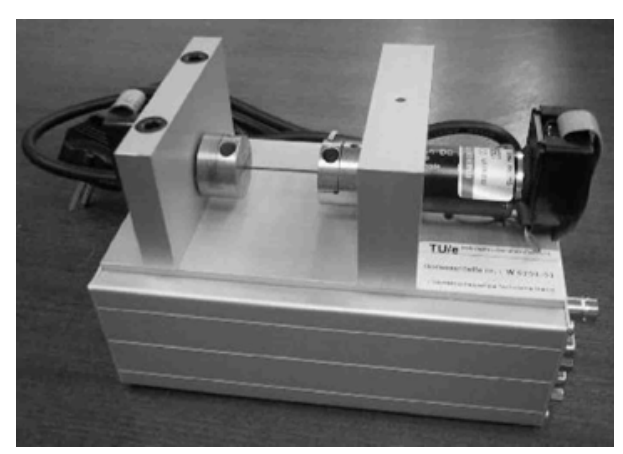

Figure 2: Two mass flexible shaft system

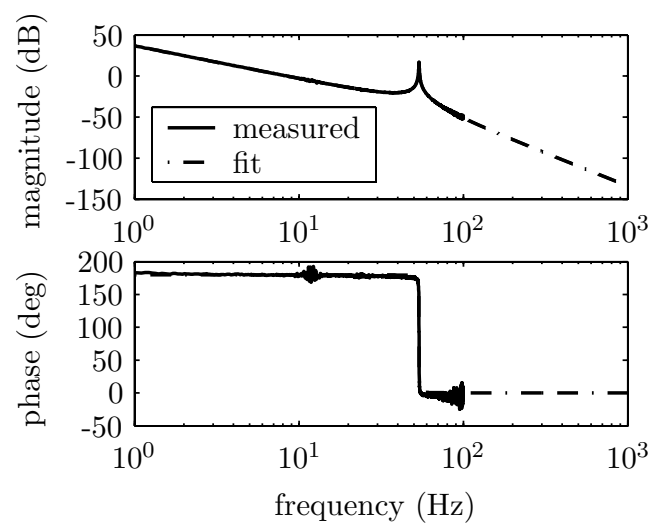

Figure 3: FRFs system and fit

\section{VALIDATION}

The validation is performed using a system consisting of two rotating masses connected through a flexible shaft as shown in Fig. 2. The measured and fitted FRFs of the system are shown in Fig. 3. The fit is used for the derivation of the learning filter $L$ and for simulation purposes. A feedback controller is designed resulting in a bandwidth of the controlled system of $5 \mathrm{~Hz}$. The sampling frequency of the controller equals $8 \mathrm{kHz}$.

The $L$ filter is based on a discrete model of $S_{P}$ using the Zero Phase Error Tracking Control (ZPETC) method [7]. A second order low-pass filter $Q$ with a cut-off frequency $f_{c}$ of $1 \mathrm{kHz}$ is used. The investigation of the influence of model uncertainties on the tracking error of ILC is performed for model gain uncertainties with and without disturbances and for model order uncertainties without additional disturbances. For all $\Delta P$ the same feedback controller $C$ is used.

\subsection{Gain uncertainties}

The gain uncertainties are modelled as additive uncertainties. Simulations are performed for both a negative model uncertainty $\Delta P=-0.1 P$ and a positive model 


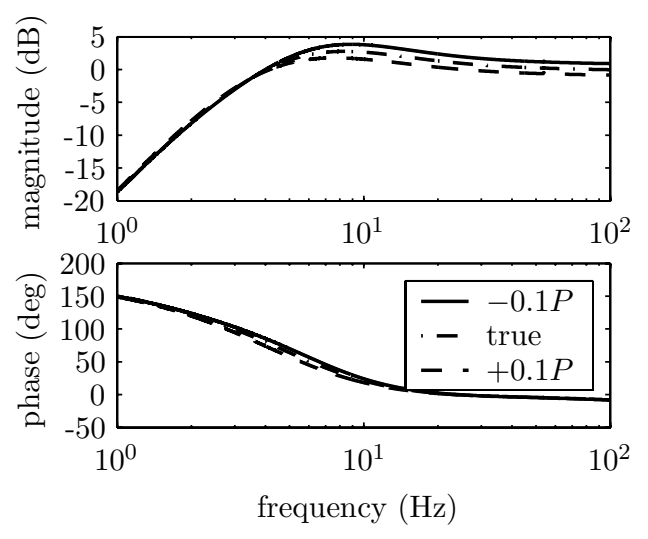

Figure 4: FRFs $S_{P} Q \tilde{L} S$ with $\Delta P$

uncertainty $\Delta P=+0.1 P$. The model uncertainties influence the learning filters $\tilde{L}$, the system used for the simulations equals the fit of the system FRF. The tracking errors without disturbances and with measurement and load disturbances will be analyzed separately.

\subsubsection{No disturbances}

For the case of no disturbances the tracking error with model uncertainties equals

$$
\begin{aligned}
e_{k, r}= & \left(1-S_{P} Q \tilde{L}\right) S r \\
& -\sum_{j=1}^{k-2} S_{P} Q\left[Q\left(1-\tilde{L} S_{P}\right)\right]^{k-j-1} \tilde{L} S r .
\end{aligned}
$$

The reference signal $r$ contains mainly frequencies below the bandwidth of $5 \mathrm{~Hz}$. The FRFs of $S_{P} Q \tilde{L} S$ with a negative and positive $\Delta P$ have below the bandwidth respectively a smaller and larger magnitude than the true fit as can be seen in Fig. 4.

The simulations with 25 iterations lead for a positive $\Delta P$ to a larger maximum absolute tracking error after convergence in comparison to the true fit and for a negative $\Delta P$ to a smaller tracking error as shown in Fig. 5. This corresponds to the results of [1] which specifies uncertainties on $S_{P}$. The presence of model uncertainties leads to a lower convergence rate as can be seen in Fig. 5 .

\subsubsection{Measurement disturbances}

The influence of measurement disturbances on the tracking error follows from (8) as

$$
\begin{aligned}
e_{k, d} & =-S d_{k}+S_{P} Q \tilde{L} S d_{k-1} \\
& +\sum_{j=1}^{k-2} S_{P} Q\left[Q\left(1-\tilde{L} S_{P}\right)\right]^{k-j-1} \tilde{L} S d_{j}
\end{aligned}
$$

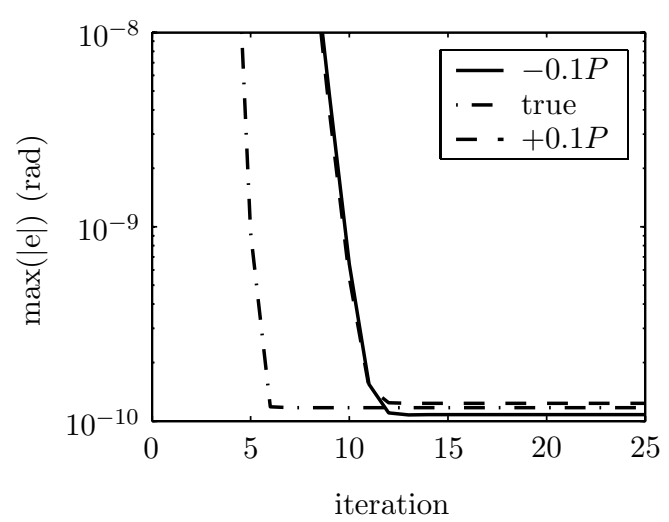

Figure 5: Maximum errors with additive $\Delta P$

Table 1: Magnitudes FRFs at $100 \mathrm{~Hz}$

\begin{tabular}{c|c|c|c}
$\Delta P$ & $|S|$ & $\left|S_{P} Q \tilde{L} S\right|$ & $\left|\sum \ldots d_{j}\right|$ \\
\hline$-0.1 P$ & 1.0009 & 1.1088 & 0.1090 \\
true & 1.0009 & 0.9963 & 0.0015 \\
$+0.1 P$ & 1.0009 & 0.9070 & 0.0918
\end{tabular}

The contribution of the disturbances $d_{k}$ to the error is not affected by the model uncertainties. The simulations are performed with harmonic measurement disturbances with constant frequency and amplitude for every iteration and varying initial phase $\phi_{0}$

$$
d_{k}=1 \cdot 10^{-2} \sin \left(2 \pi 100 t+\phi_{0}\right) .
$$

The magnitudes of the FRFs of $S$ and $S_{P} Q \tilde{L} S$ at the disturbance frequency of $100 \mathrm{~Hz}$ are given in Table 1. The magnitudes of the summation term are much smaller than the magnitudes of $S$ and $S_{P} Q \tilde{L} S$, indicating the limited influence of the disturbance signals older than two iterations.

For a simulation with two iterations the worst case occurs if the disturbances of the last two iterations have an opposite sign, i.e. an initial phase shift of $\pi \operatorname{rad}$ for the harmonic disturbances.

The initial feedforward equals the feedforward obtained from the simulations without disturbances, mak-

Table 2: Errors for measurement disturbances

\begin{tabular}{c|c|c|c}
\multirow{2}{*}{ iteration } & \multirow{2}{*}{$\Delta P$} & \multicolumn{2}{|c}{ maximum absolute error $(\mathrm{rad})$} \\
\cline { 3 - 4 } & all & $1.0009 \cdot 10^{-2}$ & $1.0006 \cdot 10^{-2}$ \\
\hline 1 & $-0.1 P$ & $2.1097 \cdot 10^{-2}$ & $2.1100 \cdot 10^{-2}$ \\
2 & true & $1.9972 \cdot 10^{-2}$ & $1.9974 \cdot 10^{-2}$ \\
& $+0.1 P$ & $1.9079 \cdot 10^{-2}$ & $1.9082 \cdot 10^{-2}$
\end{tabular}




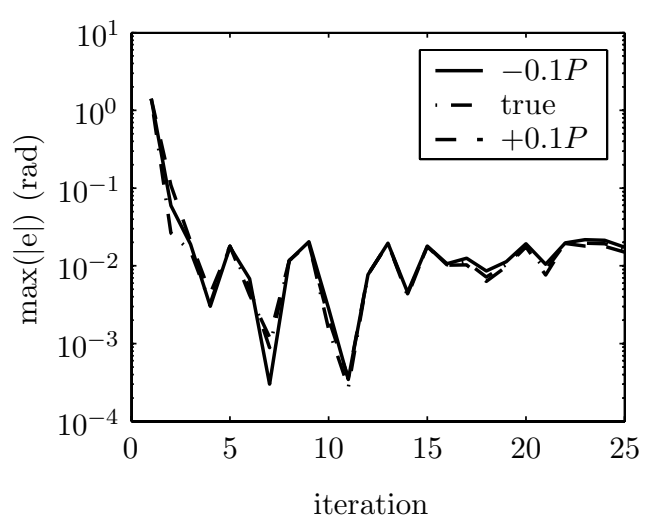

Figure 6: Maximum errors with $d_{k}$ and additive $\Delta P$

Table 3: Magnitudes FRFs at $1 \mathrm{~Hz}$

\begin{tabular}{c|c|c|c}
$\Delta P$ & $|S|$ & $\left|S_{P}^{2} Q \tilde{L}\right|$ & $\left|\sum \ldots d_{j}\right|$ \\
\hline$-0.1 P$ & 7.9526 & 8.1112 & 3.7763 \\
true & 7.9526 & 7.9879 & 0.0043 \\
$+0.1 P$ & 7.9526 & 8.3149 & 3.0766
\end{tabular}

ing the influence of the reference $r$ negligibly small. The maximum errors of the worst case simulations with the various model uncertainties can be calculated as

$$
\begin{aligned}
& \left|e_{1, d}\right|_{\max }=|S| d_{\max } \\
& \left|e_{2, d}\right|_{\max }=|S| d_{\max }+\left|S_{P} Q \tilde{L} S\right| d_{\max } .
\end{aligned}
$$

The maximum absolute calculated errors and the values found in the simulations are given in Table 2 and show a good correspondence. The presence of the disturbances in the learning process of the feedforward leads to an amplification of the tracking error. The model uncertainties affect the amount of amplification through the filter $S_{P} Q \tilde{L} S$.

The maximum absolute errors of the various iterations for simulations with 25 iterations, measurement disturbances with random phase $\phi_{0}$ and initial zero feedforward are shown in Fig. 6. The initial phases of the disturbance signals of corresponding iterations are equal. The largest maximum absolute errors of 25 iterations exceed, after the fourth iteration, the worst case simulated errors of two iterations (see Table 2) for $\Delta P=-0.1 P$ only by $2.9545 \cdot 10^{-4} \mathrm{rad}$, for the true system by $6.2886 \cdot 10^{-4} \mathrm{rad}$ and for $\Delta P=-0.1 P$ by $5.7041 \cdot 10^{-4} \mathrm{rad}$. The summation terms of (14) only have a limited influence on the tracking error.
Table 4: Errors for load disturbances

\begin{tabular}{c|c|c|c}
\multirow{2}{*}{ iteration } & \multirow{2}{*}{$\Delta P$} & \multicolumn{2}{|c}{ maximum absolute error $(\mathrm{rad})$} \\
\cline { 3 - 4 } & calculated & simulation \\
\hline 1 & all & $7.9526 \cdot 10^{-2}$ & $7.9527 \cdot 10^{-2}$ \\
\hline \multirow{3}{*}{2} & $-0.1 P$ & $1.6064 \cdot 10^{-1}$ & $1.6069 \cdot 10^{-1}$ \\
& true & $1.5941 \cdot 10^{-1}$ & $1.5941 \cdot 10^{-1}$ \\
& $+0.1 P$ & $1.6268 \cdot 10^{-1}$ & $1.6261 \cdot 10^{-1}$
\end{tabular}

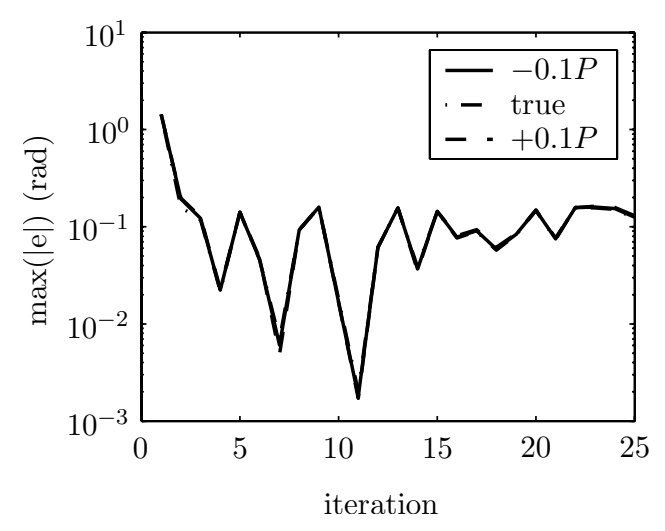

Figure 7: Maximum errors with $n_{k}$ and additive $\Delta P$

\subsubsection{Load disturbances}

The load disturbances affect the error of (8) as,

$$
\begin{aligned}
e_{k, d}= & -S_{P} n_{k}+S_{P}^{2} Q \tilde{L} n_{k-1} \\
& +\sum_{j=1}^{k-2} S_{P}^{2} Q\left[Q\left(1-\tilde{L} S_{P}\right)\right]^{k-j-1} \tilde{L} n_{j}
\end{aligned}
$$

For the simulations harmonic input disturbances with constant frequency and amplitude and varying initial phase $\phi_{0}$ are used

$$
n_{k}=1 \cdot 10^{-2} \sin \left(2 \pi 1 t+\phi_{0}\right) .
$$

The influence of the disturbances of the iteration $k$ is not affected by the model uncertainties. The magnitudes of $S_{P}$ and $S_{P}^{2} Q \tilde{L}$ at the disturbance frequency of $1 \mathrm{~Hz}$ are given in Table 3 .

As was the case for the measurement disturbances, the worst case occurs for an ILC simulation with two iterations and load disturbances if the disturbances have an opposite sign. The initial feedforward signals equal the feedforward signals obtained without disturbances. The maximum calculated absolute errors and the maximum absolute errors obtained in the simulations show a good correspondence as can be seen in Table 4. The presence of the load disturbances and model uncertainties in the learning process leads to an amplification of the tracking error. 


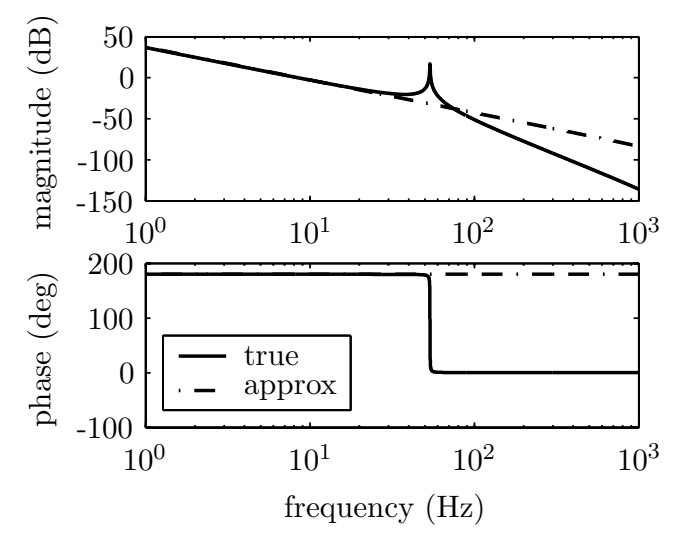

Figure 8: FRFs system and simplified model

The results of the simulations with 25 iterations, random $\phi_{0}$ and initial zero feedforward are shown in Fig. 7. The largest maximum errors show that the disturbances of iterations older than the last two do not exceed the worst case error of two iterations a lot.

\subsection{Model order uncertainties}

In practice the model of $S_{P}$ is always an approximation of the real system behavior, e.g. resonances at high frequencies are often left out. The FRFs of the fitted system and a model without resonance are shown in Fig. 8. At frequencies below the resonance the simplified model corresponds in this case to a frequencydependent negative model gain uncertainty. In order to satisfy the convergence criterion (4) a sixth order lowpass $Q$ filter with a cut-off frequency $f_{c}$ of $20 \mathrm{~Hz}$ is needed instead of a second order with $f_{c}=1000 \mathrm{~Hz}$. The simulations and experiments are performed without additional disturbances and with learning filters $\tilde{L}$ designed with the true fit and the simplified model. The change in $Q$ filter results in an increase in tracking error from $10^{-10} \mathrm{rad}$ to $10^{-4} \mathrm{rad}$ as can be seen in Fig. 5 and Fig. 9. With the simplified model a smaller error after convergence and a somewhat decreased convergence rate is obtained (see Fig. 9), this was also the case for the negative model gain uncertainty of Section 5.1. The experimental results fluctuate over the iterations due to the non-repetitive part of the error.

\section{CONCLUSION}

An expression for the tracking error of an arbitrary iteration $k$ as function of the reference signal $r$, measurement disturbances $d_{k}$ and load disturbances $n_{k}$ was derived. The influence of both disturbances and model uncertainties on the tracking error can be evaluated

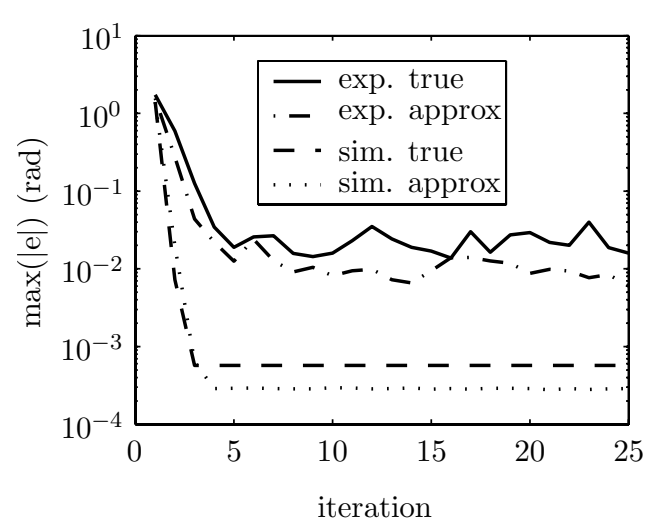

Figure 9: Maximum errors with a simplified model

with this expression. Validation is performed using simulations and experiments. The tracking error is dominated by the disturbances of the last two iterations and the model uncertainties. The model uncertainties limit the ILC performance by the need for a higher order $Q$ filter with a lower cut-off frequency.

\section{REFERENCES}

[1] B.G. Dijkstra, Iterative Learning Control with applications to a wafer-stage, Phd thesis, Delft University of Technology, September 2004.

[2] K.L. Moore, Iterative Learning Control for Deterministic Systems, Springer, Berlin, 1993.

[3] M. Norrlöf and S. Gunnarsson, "Some results on iterative learning control with disturbances," Technical report, Department of Electrical Engineering, Linköping University, Sweden, March 1998.

[4] M. Norrlöf and S. Gunnarsson, "Disturbance aspects of iterative learning control," Engineering Applications of Artificial Intelligence, Elsevier, vol. 14, pp. 87-94, 2001.

[5] S. Panzieri and G. Ulivi, "Disturbance rejection of iterative learning control applied to trajectory tracking for a flexible manipulator," 3rd European Control Conference, pp. 2374-2379, 1995.

[6] M. Steinbuch and M.J.G. van de Molengraft, "Iterative learning control of industrial motion systems," 1st IFAC conference on Mechatronic Systems, pp. 967-972, 2000.

[7] M. Tomizuka, "Zero phase error tracking algorithm for digital control," ASME Journal of Dynamic Systems, Measurement and Control, vol. 109, pp. 65-68, 1987. 